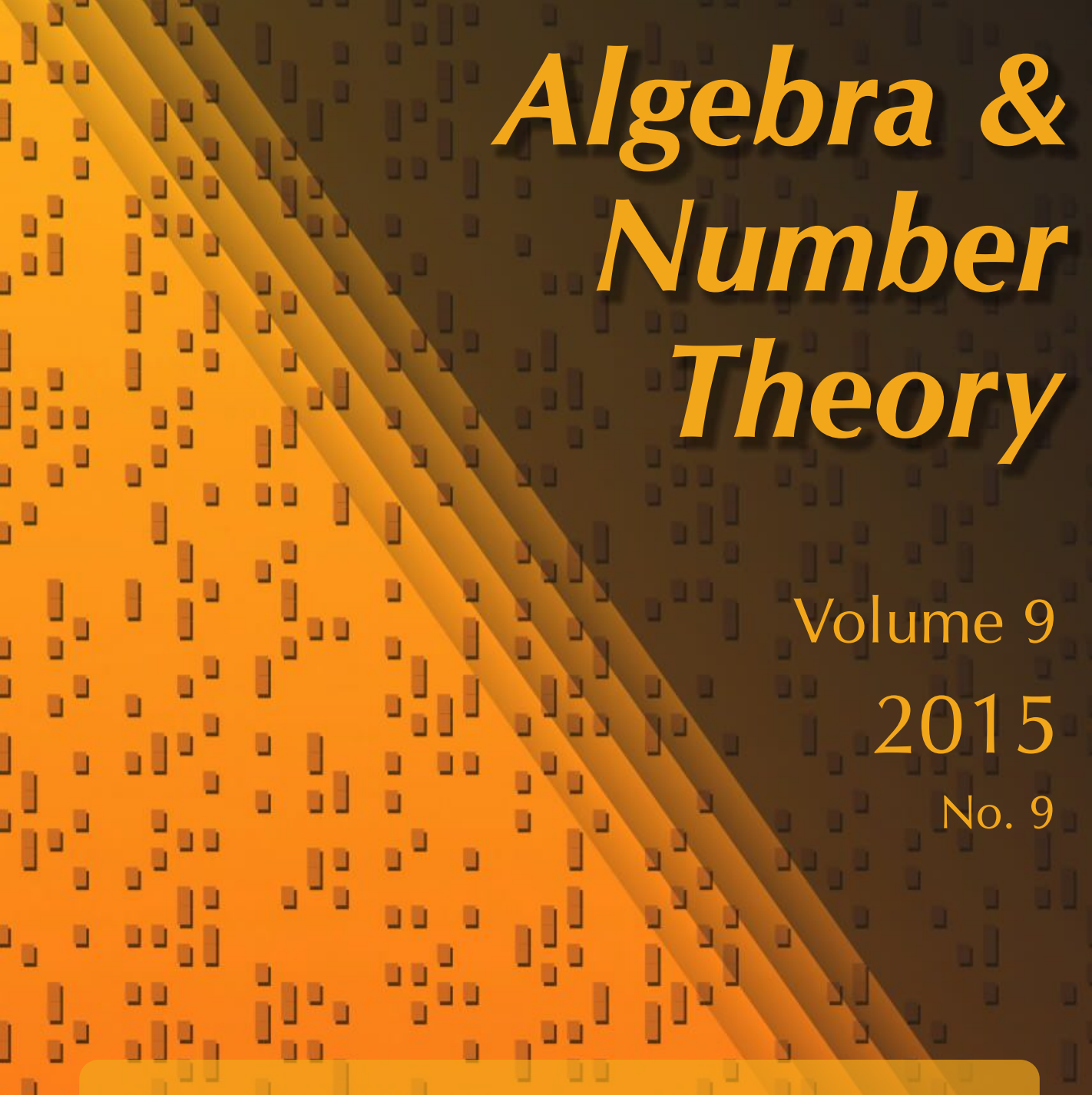

Classifying orders in the Sklyanin algebra

Daniel Rogalski, Susan J. Sierra and J. Toby Stafford

」

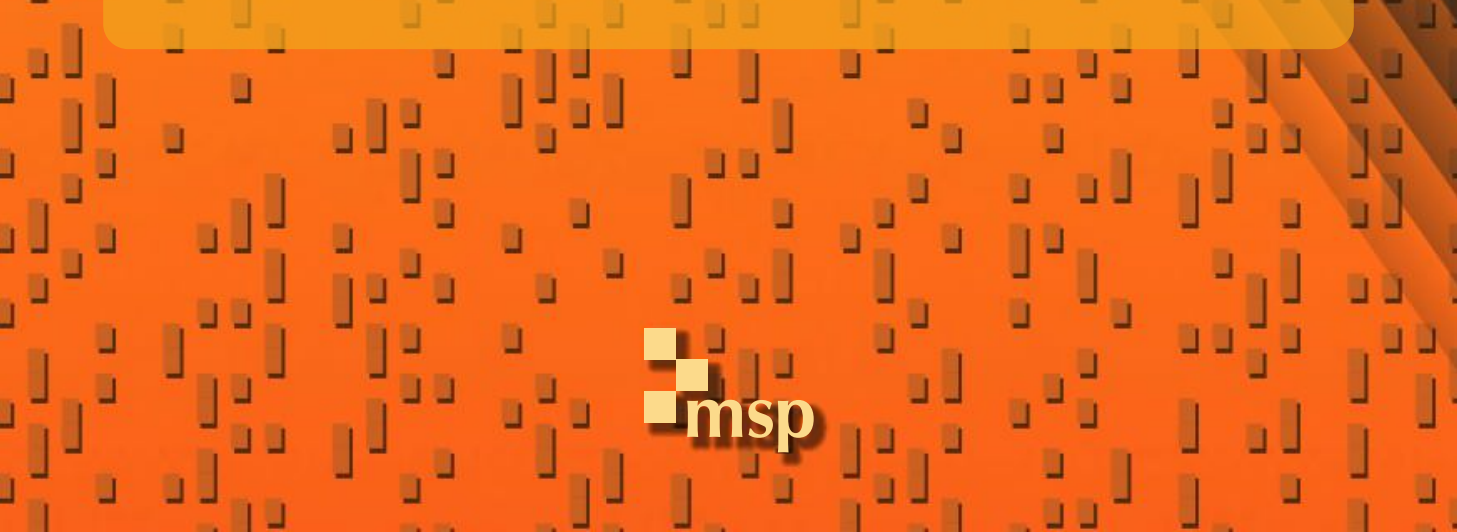




\title{
Classifying orders in the Sklyanin algebra
}

\author{
Daniel Rogalski, Susan J. Sierra and J. Toby Stafford
}

Let $S$ denote the 3-dimensional Sklyanin algebra over an algebraically closed field $\mathbb{k}$ and assume that $S$ is not a finite module over its centre. (This algebra corresponds to a generic noncommutative $\mathbb{P}^{2}$.) Let $A=\bigoplus_{i \geq 0} A_{i}$ be any connected graded $\mathbb{k}$-algebra that is contained in and has the same quotient ring as a Veronese ring $S^{(3 n)}$. Then we give a reasonably complete description of the structure of $A$. This is most satisfactory when $A$ is a maximal order, in which case we prove, subject to a minor technical condition, that $A$ is a noncommutative blowup of $S^{(3 n)}$ at a (possibly noneffective) divisor on the associated elliptic curve $E$. It follows that $A$ has surprisingly pleasant properties; for example, it is automatically noetherian, indeed strongly noetherian, and has a dualising complex.

1. Introduction 2056

2. Basic results 2061

3. Curves 2069

4. Right ideals of $T$ and the rings $T(\boldsymbol{d}) \quad 2075$

5. An equivalent $T(\boldsymbol{d}) \quad 2079$

6. On endomorphism rings of $T(\boldsymbol{d})$-modules 2088

7. The structure of $g$-divisible orders 2095

8. Sporadic ideals and $g$-divisible hulls 2101

9. Arbitrary orders 2109

10. Examples 2111

Acknowledgements 2116

Index of notation $\quad 2117$

References $\quad 2117$

Rogalski was partially supported by NSF grants DMS-0900981 and DMS-1201572. Sierra was partially supported by an NSF Postdoctoral Research Fellowship, grant DMS-0802935. Stafford was a Royal Society Wolfson Research Merit Award Holder.

MSC2010: primary 14A22; secondary 16P40, 16W50, 16S38, 14H52, 16E65, $18 \mathrm{E} 15$.

Keywords: noncommutative projective geometry, noncommutative surfaces, Sklyanin algebras, noetherian graded rings, noncommutative blowing-up. 


\section{Introduction}

Noncommutative (projective) algebraic geometry has been very successful in using techniques and intuition from algebraic geometry to study noncommutative graded algebras, and many classes of algebras have been classified using these ideas. In particular, noncommutative irreducible curves (or connected graded domains of Gelfand-Kirillov dimension 2) have been classified [Artin and Stafford 1995] as have large classes of noncommutative irreducible surfaces (or connected graded noetherian domains of Gelfand-Kirillov dimension 3).

Indeed, the starting point of this subject was really the classification by Artin, Tate, and Van den Bergh [Artin et al. 1990; 1991] of noncommutative projective planes (noncommutative analogues of a polynomial ring $\mathbb{k}[x, y, z]$ ). The generic example here is the Sklyanin algebra

$$
S=\operatorname{Skl}(a, b, c)=\mathbb{k}\left\{x_{1}, x_{2}, x_{3}\right\} /\left(a x_{i} x_{i+1}+b x_{i+1} x_{i}+c x_{i+2}^{2}: i \in \mathbb{Z}_{3}\right),
$$

where $(a, b, c) \in \mathbb{P}^{2} \backslash \mathscr{Y}$ for a (known) finite set $\mathscr{Y}$. The geometric methods of [Artin et al. 1990] were necessary to understand this algebra. See [Stafford and Van den Bergh 2001] for a survey of many of these results.

In the other direction, one would like to classify all noncommutative surfaces, and a programme for this has been suggested by Artin [1997]. This paper completes a significant case of this programme by classifying the graded noetherian orders contained in the Sklyanin algebra. In this introduction we will first describe our main results and then discuss the historical background and give an idea of the proofs.

The main results. Fix a Sklyanin algebra $S=\operatorname{Skl}(a, b, c)$ defined over an algebraically closed base field $\mathbb{k}$. For technical reasons we mostly work inside the 3-Veronese ring $T=S^{(3)}$; thus $T=\bigoplus T_{n}$ with $T_{n}=S_{3 n}$ for each $n$, under the natural graded structure of $S$. The difference between these algebras is not particularly significant; for example, the quotient category qgr- $T$ of graded noetherian right $T$-modules modulo those of finite length, is equivalent to qgr- $S$. Then $T$ contains a canonical central element $g \in T_{1}=S_{3}$ such that the factor $B=T / g T$ is a TCR or twisted homogeneous coordinate ring $B=B(E, M, \tau)$ of an elliptic curve $E$. Here $\mathcal{M}$ is a line bundle of degree 9 and $\tau \in \operatorname{Aut}_{\mathbb{k}}(E)$ (see Section 2 for the definition). We assume throughout the paper that $|\tau|=\infty$; equivalently, that $T$ is not a finite module over its centre.

Our main results are phrased in terms of certain blowups $T(\boldsymbol{d}) \subset T$, where $\boldsymbol{d}$ is a divisor on $E$. These are discussed in more detail later in this introduction. Here we will just note that, when $\boldsymbol{p}$ is a closed point of $E$, the blowup $T(\boldsymbol{p})$ is the subring of $T$ generated by those elements $x \in T_{1}$ whose images in $T / g T$ vanish at $\boldsymbol{p}$. For an effective divisor $\boldsymbol{d}$ (always of degree at most 8), $T(\boldsymbol{d})$ has properties similar to those of a (commutative) anticanonical homogeneous coordinate ring of the blowup 
of $\mathbb{P}^{2}$ along the divisor $\boldsymbol{d}$. However, we also need algebras that should be considered as blowups $T\left(\boldsymbol{d}^{\prime}\right)$ of $T$ at noneffective divisors of the form $\boldsymbol{d}^{\prime}=\boldsymbol{x}-\boldsymbol{y}+\tau^{-1}(\boldsymbol{y})$, where $\boldsymbol{x}$ and $\boldsymbol{y}$ are effective divisors on $E, 0 \leq \operatorname{deg} \boldsymbol{d}^{\prime} \leq 8$ and certain combinatorial conditions hold (see Definition 7.1 for the details). Such a divisor will be called virtually effective.

Given domains $U, U^{\prime}$ with the same Goldie quotient ring $Q(U)=Q\left(U^{\prime}\right)=Q$, we say that $U$ and $U^{\prime}$ are equivalent orders if $a U b \subseteq U^{\prime}$ and $a^{\prime} U^{\prime} b^{\prime} \subseteq U$ for some $a, b, a^{\prime}, b^{\prime} \in Q \backslash\{0\}$. If $Q(U)=Q(V)$ for some ring $V \supseteq U$, then $U$ is called a maximal $V$-order if there exists no ring $U^{\prime}$ equivalent to $U$ such that $U \subsetneq U^{\prime} \subseteq V$. When $V=Q(U), U$ is simply termed a maximal order. These can be regarded as the appropriate noncommutative analogues of integrally closed domains. The algebra $T$ is a maximal order. When $Q_{\mathrm{gr}}(U)=Q_{\mathrm{gr}}(T)$ the concepts of maximal orders and maximal $T$-orders are essentially the only cases that will concern us and, as the next result shows, they are closely connected.

In this result, an $\mathbb{N}$-graded $\mathbb{k}$-algebra $A=\bigoplus_{n \geq 0} A_{n}$ is called connected graded (cg) if $A_{0}=\mathbb{k}$ and $\operatorname{dim}_{\mathbb{k}} A_{n}<\infty$ for all $n$. Also, for a cg algebra $U \subseteq T$, we write $\bar{U}=(U+g T) / g T$.

Proposition 1.1 (combine Theorem 8.11 with Proposition 6.4). Let $U$ be a cg maximal $T$-order, such that $\bar{U} \neq \mathbb{k}$. Then there exists a unique maximal order $F=F(U) \supseteq U$ equivalent to $U$. Moreover, $F$ is a finitely generated $U$-module with $\operatorname{GKdim}_{U}(F / U) \leq 1$.

We remark that there do exist graded maximal $T$-orders $U$ with $U \neq F(U)$ (see Proposition 10.3).

Our results are most satisfactory for maximal $T$-orders, and our main result is the following complete classification of such algebras.

Theorem 1.2 (Theorem 8.11). Let $U$ be a cg maximal $T$-order with $\bar{U} \neq \mathbb{k}$. Then there exists a virtually effective divisor $\boldsymbol{d}^{\prime}=\boldsymbol{d}-\boldsymbol{y}+\tau^{-1}(\boldsymbol{y})$ with $\operatorname{deg}\left(\boldsymbol{d}^{\prime}\right) \leq 8$ such that the associated maximal order $F(U)$ is a blowup $F(U)=T\left(\boldsymbol{d}^{\prime}\right)$ of $T$ at $\boldsymbol{d}^{\prime}$.

Remarks 1.3. (1) Although in this introduction we are restricting our attention to the Sklyanin algebra $S=S k l$, this theorem and indeed all the results of this paper are proved simultaneously for certain related algebras; see Assumption 2.1 and Examples 2.2 for the details.

(2) Theorem 1.2 is actually proved in the context of graded maximal $T^{(n)}$-orders, but as that result is a little more complicated to state, the reader is referred to Theorem 8.11 for the details.

(3) The assumption that $\bar{U} \neq \mathbb{k}$ in the theorem is annoying but necessary (see Example 10.8). It can be bypassed at the expense of passing to a Veronese ring and 
then regrading the algebra. However, the resulting theorems are not as strong as Theorem 1.2 (see Section 9 for the details).

One consequence of Theorem 1.2 is that maximal $T$-orders have very pleasant properties. The undefined terms in the next result are standard concepts and are defined in the body of the paper.

Corollary 1.4. Let $U$ and $F=F(U)=T\left(\boldsymbol{d}^{\prime}\right)$ be as in Theorem 1.2.

(1) (Proposition 2.9 and Theorem 8.11(1)) Both $U$ and $F$ are finitely generated $\mathbb{k}$-algebras and are strongly noetherian: in other words, $U \otimes_{\mathbb{k}} C$ and $F \otimes_{\mathbb{k}} C$ are noetherian for any commutative, noetherian $\mathbb{k}$-algebra $C$.

(2) (Corollary 8.12) Both $U$ and $F$ satisfy the Artin-Zhang $\chi$ conditions, have finite cohomological dimension and possess balanced dualising complexes.

(3) (Proposition 4.10 and Example 10.4) If $F$ is the blowup at an effective divisor then $U=F$. In this case $F$ also satisfies the Auslander-Gorenstein and CohenMacaulay conditions. These conditions do not necessarily hold when $\boldsymbol{d}^{\prime}$ is virtually effective.

In the other direction, we prove:

Theorem 1.5 (Theorem 7.4(3)). For any virtually effective divisor $\boldsymbol{d}^{\prime}$ there exists a blowup of $T$ at $\boldsymbol{d}^{\prime}$ in the sense described above.

The fact that $U$ is automatically noetherian in Theorem 1.2 is one of the result's most striking features. In general, nonnoetherian graded subalgebras of $T$ can be rather unpleasant and so, in order to classify reasonable classes of nonmaximal orders in $T$, we make a noetherian hypothesis. Given a connected graded noetherian algebra $U$, one can easily obtain further noetherian rings by taking Veronese rings, idealiser subrings $\llbracket(J)=\{\theta \in U: \theta J \subseteq J\}$ for a right ideal $J$ of $U$, or equivalent orders $U^{\prime} \subseteq U$ containing an ideal $K$ of $U$. We show that this suffices:

Corollary 1.6 (Corollary 9.5). Let $U$ be a cg noetherian subalgebra of $T$ with $Q_{\mathrm{gr}}(U)=Q_{\mathrm{gr}}\left(T^{(n)}\right)$ for some $n$. Assume that $\bar{U} \neq \mathbb{k}$ (as in Remarks 1.3, this can be assumed at the expense of taking a Veronese ring and regrading).

Then $U$ can be obtained from some virtual blowup $R=T\left(\boldsymbol{d}^{\prime}\right)$ by a combination of Veronese rings, idealisers and equivalent orders $K \subseteq U \subseteq V$, where $K$ is an ideal of $V$ with $\operatorname{GKdim}(V / K) \leq 1$.

History. We briefly explain the history behind these results and their wider relevance. As we mentioned earlier, noncommutative curves and noncommutative analogues of the polynomial ring $\mathbb{k}[x, y, z]$ have been classified. Motivated by these results, Artin suggested a program for classifying all noncommutative surfaces, but in order to outline this program we need some notation. 
Given a cg domain $A$ of finite Gelfand-Kirillov dimension, one can invert the nonzero homogeneous elements to obtain the graded quotient ring $Q_{\mathrm{gr}}(A) \cong$ $D\left[t, t^{-1} ; \alpha\right]$, for some automorphism $\alpha$ of the division ring $D=Q(A)_{0}=D_{\mathrm{gr}}(A)$. This division ring will be called the function skewfield of $A$.

Let $A$ be a noetherian, cg $\mathbb{k}$-algebra. A useful intuition is to regard qgr- $A$ as the coherent sheaves over the (nonexistent) noncommutative projective scheme $\operatorname{Proj}(A)$, although we will slightly abuse notation by regarding qgr- $A$ itself as that scheme. Under this intuition, a noncommutative surface is qgr- $A$ for a noetherian $\operatorname{cg}$ domain $A$ with $\mathrm{GKdim} A=3$. (In fact, one should probably weaken this last condition to the assumption that $D_{\mathrm{gr}}(A)$ has lower transcendence degree two in the sense of [Zhang 1998], but that is not really relevant here.) There are strong arguments for saying that noncommutative projective planes are the categories qgr- $A$, as $A$ ranges over the Artin-Schelter regular rings of dimension 3 with the Hilbert series $(1-t)^{-3}$ of a polynomial ring in three variables (see [Stafford and Van den Bergh 2001, §11.2] for more details). These are the algebras classified in [Artin et al. 1990] and for which the Sklyanin algebra $S=S(a, b, c)$ is the generic example. Van den Bergh [2011; 2012] has similarly classified noncommutative analogues of quadrics and related surfaces.

Artin's Conjectures 1.7. Artin conjectured that the only function skewfields of noncommutative surfaces are the following:

(i) division rings $D$ finite-dimensional over their centres $F=Z(D)$, which are then fields of transcendence degree two;

(ii) division rings of fractions $D$ of Ore extensions $\mathbb{k}(X)[z ; \sigma, \delta]$ for some curve $X$, where $D$ is not a finite module over its centre; and

(iii) the function skewfield $D=D_{\mathrm{gr}}(S)$ of a Sklyanin algebra $S=S(a, b, c)$, where $S$ is not a finite module over its centre.

Artin then asked for a classification of the noncommutative surfaces qgr- $A$ within each birational class; that is, the cg noetherian algebras $A$ with $D_{\mathrm{gr}}(A)$ being a fixed division ring from this list.

The case of Artin's programme when $D=\mathbb{k}(Y)$ is the function field of a surface and GKdim $A=3$ has been completed in [Rogalski and Stafford 2009; Sierra 2011] (if one strays from algebras of Gelfand-Kirillov dimension 3, then things become more complicated, as [Rogalski and Sierra 2012] shows). As explained earlier, in this paper we are interested in the other extreme, that of case (iii) from Artin's list.

The first main results in this direction come from [Rogalski 2011], of which this paper is a continuation. In particular, [ibid., Theorem 1.2] shows that the maximal orders $U \subseteq T=S^{(3)}$ that have $Q_{\mathrm{gr}}(U)=Q_{\mathrm{gr}}(T)$ and are generated in degree one are just the blowups $T(\boldsymbol{d})$ for an effective divisor $\boldsymbol{d}$ on $E$ with $\operatorname{deg}(\boldsymbol{d}) \leq 7$. We remark 
that in this case $T(\boldsymbol{d})$ is simply the subalgebra of $T$ generated by those elements of $T_{1}$ whose images in $T / g T$ vanish on $\boldsymbol{d}$. As such, $T(\boldsymbol{d})$ is quite similar to a commutative blowup and qgr- $T(\boldsymbol{d})$ also coincides with the more categorical version of a blowup in [Van den Bergh 2001]. In this paper we will also need $T(\boldsymbol{d})$ when $\operatorname{deg}(\boldsymbol{d})=8$, and this is harder to describe as it is not generated in degree one. Its construction and basic properties are described in the companion paper [RSS 2015].

The proofs. For simplicity we assume here that $U$ is a cg subalgebra of $T$ with $Q_{\mathrm{gr}}(U)=Q_{\mathrm{gr}}(T)$.

A key strategy in the description of the Sklyanin algebra $S$, and in the classification of noncommutative projective planes in [Artin et al. 1990], was to understand the factor ring $S / g S$, where $g \in S_{3}=T_{1}$ is the central element mentioned earlier. Indeed, one of the main steps in that paper was to show that $S / g S \cong B(E, \mathscr{L}, \sigma)$ for the appropriate $\mathscr{L}$ and $\sigma$. We apply a similar strategy. The nicest case is when $U \subseteq T$ is $g$-divisible in the sense that $g \in U$ and $U \cap g T=g U$. In particular, $\bar{U}=U / g U$ is then a subalgebra of $\bar{T}$ with $\operatorname{GKdim}(\bar{U})=2$. As such $\bar{U}$ and hence $U$ are automatically noetherian (see Proposition 2.9). Much of this paper concerns the classification of $g$-divisible algebras $U$, and the starting point is the following result.

Theorem 1.8 (Theorem 5.24). Let $U$ be a g-divisible subalgebra of $T$ such that $Q_{\mathrm{gr}}(U)=Q_{\mathrm{gr}}(T)$. Then $U$ is an equivalent order to some blowup $T(\boldsymbol{d})$ at an effective divisor $\boldsymbol{d}$ on $E$ with $\operatorname{deg} \boldsymbol{d} \leq 8$.

It follows easily from this result that a g-divisible maximal $T$-order $U$ equals $\operatorname{End}_{T(\boldsymbol{d})}(M)$ for some finitely generated right $T$-module $M$ (see Corollary 6.6). When $U$ is $g$-divisible, the rest of the proof of Theorem 1.2 amounts to showing that, up to a finite-dimensional vector space, $\bar{U}=B\left(E, M\left(-d^{\prime}\right), \tau\right)$, for some virtually effective divisor $\boldsymbol{d}^{\prime}=\boldsymbol{d}-\boldsymbol{y}+\tau^{-1}(\boldsymbol{y})$ (see Theorem 6.7). This is also the key property in the definition of a blowup at such a divisor (see Definitions 6.9 and 7.1 for more details).

Now suppose that $U$ is not necessarily $g$-divisible and set $C=U\langle g\rangle$ with $g$ divisible hull

$$
\widehat{C}=\left\{\theta \in T: g^{m} \theta \in C \text { for some } m \geq 0\right\} .
$$

The remaining step in the proof of Theorem 1.2 is to show that $U, C$ and $\widehat{C}$ are equivalent orders. This in turn follows from the following fact. Let $V$ be $a$ graded subalgebra of $T$ with $g \in V$ and $Q_{\mathrm{gr}}(V)=Q_{\mathrm{gr}}(T)$. Then $V$ has a minimal sporadic ideal in the sense that $V$ has a unique ideal I minimal with respect to $\operatorname{GKdim}(V / I) \leq 1$ and $V / I$ being $g$-torsionfree (see Corollary 8.8).

Further results. The $g$-divisible subalgebras of $T$ are closely related to subalgebras of the (ungraded) localised ring $T^{\circ}=T\left[g^{-1}\right]_{0}$. The algebra $T^{\circ}$ is a hereditary noetherian domain of GK-dimension 2 and can be thought of as a noncommutative 
coordinate ring of the affine space $\mathbb{P}^{2} \backslash E$. By [RSS 2014], any subalgebra of $T^{\circ}$ is noetherian and so the algebras $U^{\circ}=U\left[g^{-1}\right]_{0} \subseteq T^{\circ}$ give a plentiful supply of noetherian domains of GK-dimension 2. All the above results have parallel versions for orders in $T^{\circ}$. For example:

Corollary 1.9 (Corollary 7.10 and Corollary 8.5). Let $A$ be a subalgebra of $T^{\circ}$ with $Q(A)=Q\left(T^{\circ}\right)$.

(1) The algebra A has finitely many prime ideals and DCC on ideals.

(2) If $A$ is a maximal $T^{\circ}$-order then $A=T\left(\boldsymbol{d}^{\prime}\right)^{\circ}$ for some virtually effective divisor $\boldsymbol{d}^{\prime}$.

Organisation of the paper. In Section 2 we prove basic technical results, including the important, though easy, fact that any $g$-divisible subalgebra of $T$ is strongly noetherian (see Proposition 2.9). Section 3 is devoted to studying finitely generated graded orders in $\mathbb{k}(E)[t ; \tau]$. The main result (Theorem 3.1) shows that any such order is (up to finite dimension) an idealiser in a twisted homogeneous coordinate ring. This improves on one of the main results from [Artin and Stafford 1995] and has useful applications to the study of point modules over such an algebra. Section 4 incorporates needed results from [RSS 2015] about right ideals of $T$ and the blowups $T(\boldsymbol{d})$ at effective divisors.

Sections 5-7 are devoted to $g$-divisible algebras in $T$. The main result of Section 5 is Theorem 1.8 from above. Section 6 is concerned with the structure of $V=\operatorname{End}_{T(\boldsymbol{d})}(M)$, where $M \subset T$ is a reflexive $T(\boldsymbol{d})$-module and $\boldsymbol{d}$ is effective. Most importantly, Theorem 6.7 describes the factor $V / g V$. Section 7 pulls these results together, proves Theorem 1.5 for $g$-divisible algebras and draws various conclusions.

In Section 8 we show that various algebras have minimal sporadic ideals. This is then used to complete the proof of Theorem 1.2. Section 9 studies subalgebras of the Veronese rings $T^{(m)}$ and algebras $U$ with $\bar{U}=\mathbb{k}$. We apply this to prove Corollary 1.6. Finally, Section 10 is devoted to examples. At the end of the paper we also provide an index of notation.

\section{Basic results}

In this section we collect the basic definitions and results that will be used throughout the paper.

Throughout the paper $k$ is an algebraically closed field and all rings will be $\mathbb{k}$ algebras. If $X$ is a projective $\mathbb{k}$-scheme, $\mathscr{L}$ is an invertible sheaf on $X$, and $\sigma: X \rightarrow X$ is an automorphism, then there is a TCR or twisted homogeneous coordinate ring $B=B(X, \mathscr{L}, \sigma)$ associated to this data and defined as follows. Write $\mathscr{F}^{\sigma}=\sigma^{*}(\mathscr{F})$ 
for a pullback of a sheaf $\mathscr{F}$ on $X$ and set $\mathscr{F}_{n}=\mathscr{F} \otimes \mathscr{F}^{\sigma} \otimes \cdots \otimes \mathscr{F}^{n-1}$ for $n \geq 1$. Then

$$
B=\bigoplus_{n \geq 0} H^{0}\left(X, \mathscr{L}_{n}\right), \quad \text { with product } x * y=x \otimes\left(\sigma^{m}\right)^{*}(y) \text { for } x \in B_{m}, y \in B_{n} .
$$

In this paper $X=E$ will usually be a smooth elliptic curve, and a review of some of the important properties of $B(E, \mathscr{L}, \sigma)$ in this case can be found in [Rogalski 2011]. It is well known, going back to [Artin et al. 1990], that much of the structure of the Sklyanin algebra $S$ is controlled by the factor $\operatorname{ring} S / g S \cong B(E, \mathscr{L}, \sigma)$, and this in turn can be analysed geometrically.

In fact, there are several different families of Sklyanin algebras, and we first set up a framework which will allow our results to apply to subalgebras of any of these (and, indeed, more generally). Recall that for an $\mathbb{N}$-graded ring $R=\bigoplus_{n \geq 0} R_{n}$ the $d$-th Veronese ring, for $d \geq 1$, is $R^{(d)}=\bigoplus_{n \geq 0} R_{n d}$. Usually this is graded by setting $R_{n}^{(d)}=R_{n d}$. However, we will sometimes want to regard $R^{(d)}$ as a graded subring of $R$, in which case each $R_{n d}$ maintains its degree $n d$; we will call this the unregraded Veronese ring. In this paper it will be easier to work with the 3-Veronese ring of the Sklyanin $T=S^{(3)}=\bigoplus_{n \in \mathbb{Z}} T_{n}$, largely because this ensures that the canonical central element $g$ lies in $T_{1}$. Similar comments will apply to the other families, and so in the body of the paper we will work with algebras satisfying the following hypotheses.

Assumption 2.1. Let $T$ be a cg $\mathbb{k}$-algebra which is a domain with a central element $0 \neq g \in T_{1}$, such that there is a graded isomorphism $T / g T \cong B=B(E, \mu, \tau)$ for a smooth elliptic curve $E$, invertible sheaf $\mathcal{M}$ with $\mu=\operatorname{deg} M \geq 2$, and infinite-order automorphism $\tau$. Such a $T$ is called an elliptic algebra of degree $\mu$.

This assumption holds throughout the paper. In the language of [Van den Bergh 2001], the assumption can be interpreted geometrically to say that the surface qgr- $T$ contains the commutative elliptic curve qgr- $B \simeq \operatorname{coh} E$ as a divisor. We will need stronger conditions on $T$ in the main results of Section 8 (see Assumption 8.2).

Examples 2.2. The hypotheses of Assumption 2.1 are satisfied in a number of examples, in particular for Veronese rings of the following types of Sklyanin algebras.

(1) Let $S$ be the quadratic Sklyanin algebra

$$
S(a, b, c)=\mathbb{k}\left\{x_{0}, x_{1}, x_{2}\right\} /\left(a x_{i} x_{i+1}+b x_{i+1} x_{i}+c x_{i+2}^{2}: i \in \mathbb{Z}_{3}\right),
$$

for appropriate $[a, b, c] \in \mathbb{P}_{k}^{2}$, and let $T=S^{(d)}$ for $d=3$.

(2) Let $S$ be the cubic Sklyanin algebra

$$
S(a, b, c)=\mathbb{k}\left\{x_{0}, x_{1}\right\} /\left(a x_{i+1}^{2} x_{i}+b x_{i+1} x_{i} x_{i+1}+a x_{i} x_{i+1}^{2}+c x_{i}^{3}: i \in \mathbb{Z}_{2}\right),
$$

for appropriate $[a, b, c] \in \mathbb{P}_{k}^{2}$ and let $T=S^{(d)}$, for $d=4$. 
(3) Let $x$ have degree 1 and $y$ degree 2, and set

$$
S=S(a, b, c)=\mathbb{k}\{x, y\} /\left(a y^{2} x+c y x y+a x y^{2}+b x^{5}, a x^{2} y+c x y x+a y x^{2}+b y^{2}\right),
$$

for appropriate $[a, b, c] \in \mathbb{P}_{k}^{2}$, and let $T=S^{(d)}$, for $d=6$.

(4) There are other examples satisfying these hypotheses; for example, take $T=B(E, M, \tau)[g]$, where $\mathcal{M}$ is an invertible sheaf on the elliptic curve $E$ with $\operatorname{deg} M \geq 2$ and $|\tau|=\infty$.

The detailed properties of the examples above can be found in [Artin et al. 1990; 1991; Stephenson 1997]. In particular, the restrictions on the parameters $\{a, b, c\}$ in (1-3) are determined as follows. In each case, there exists a central element $g \in S_{d}$ such that $S / g S \cong B=B(E, \mathscr{L}, \sigma)$, for some $\mathscr{L}$ and $\sigma$. This factor ring also determines the Sklyanin algebra, since $g$ is the unique relation for $B$ of degree $d$ [Artin et al. 1990, Theorem 6.8(1); Stephenson 1997, Theorem 4.1]. The requirements on $\{a, b, c\}$ are that $E$ is an elliptic curve and that $|\sigma|=\infty$. Explicit criteria on the parameters are known for $E$ to be an elliptic curve but not for $|\sigma|=\infty$; nevertheless, this will be the case when the parameters are generic. In these examples, $\operatorname{deg} \mathscr{L}=3,2,1$, respectively, and hence $T / g T \cong B\left(E, M, \sigma^{d}\right)$, where $\mu=\mathscr{L}_{d}$ has degree $\mu=d \cdot(\operatorname{deg} \mathscr{L})=9,8,6$, respectively.

Notation 2.3. All algebras $A$ considered in this paper are domains of finite GelfandKirillov dimension, written $\operatorname{GKdim}(A)$. If $A$ is graded, then the set $\mathscr{C}$ of nonzero homogeneous elements therefore forms an Ore set (see [McConnell and Robson 2001, Corollary 8.1.21] and [Năstăsescu and van Oystaeyen 1982, C.I.1.6]). By [ibid., A.14.3], the localisation $Q_{\mathrm{gr}}(A)=A^{\mathscr{C}^{-1}}$ is a graded division ring in the sense that $Q_{\mathrm{gr}}(A)$ is an Ore extension $Q_{\mathrm{gr}}(A)=D\left[z, z^{-1} ; \alpha\right]$ of a division ring $D$ by an automorphism $\alpha$; thus $z d=d^{\alpha} z$ for all $d \in D$. The algebra $D$ will be denoted $D=D_{\mathrm{gr}}(A)$ and called the function skewfield of $A$, while $Q_{\mathrm{gr}}(A)$ will be called the graded quotient ring of $A$.

Notation 2.4. For the most part, the algebras $A$ considered in this paper will be connected graded, in which case we usually work in the category Gr- $A$ of $\mathbb{Z}$-graded right $A$-modules, with homomorphisms $\operatorname{Hom}_{\mathrm{Gr}-A}(M, N)$ being graded of degree zero. In particular, an isomorphism of graded modules or rings will be assumed to be graded of degree zero, unless otherwise stated. The category of noetherian graded right $A$-modules will be written gr- $A$, while the category of ungraded modules will be written Mod- $A$, and we reserve the term $\operatorname{Hom}(M, N)=\operatorname{Hom}_{A}(M, N)$ for homomorphisms in the ungraded category. For $M, N \in \mathrm{Gr}-A$, the shift $M[n]$ is defined by $M[n]=\bigoplus M[n]_{i}$ for $M[n]_{i}=M_{n+i}$. Similar comments apply to $\operatorname{Ext}_{\mathrm{Gr}-A}$ and $\operatorname{Ext}_{A}$ as well as to $\operatorname{End}_{A}(M)=\operatorname{Hom}_{A}(M, M)$. If fd- $A$ denotes the category of finite-dimensional (right) $A$-modules, then we write qgr- $A$ for the quotient category gr- $A$ /fd- $A$. Similarly, $A$-qgr $=A$-gr $/ A$-fd is the quotient category of noetherian 
graded left modules modulo finite-dimensional modules. The basic properties of this construction can be found in [Artin and Zhang 1994].

Notation 2.5. Write $T_{(g)}$ for the homogeneous localisation of $T$ at the completely prime ideal $g T$; thus $T_{(g)}=T \mathscr{C}^{-1}$ for $\mathscr{C}$ the set of homogeneous elements in $T \backslash g T$. Note that $T_{(g)} / g T_{(g)} \cong Q_{\mathrm{gr}}(B)=\mathbb{k}(E)\left[t, t^{-1} ; \tau\right]$, a ring of twisted Laurent polynomials over the function field of $E$. In particular, $T_{(g)} / g T_{(g)}$ is a graded division ring and by [Goodearl and Warfield 1989, Exercise 1Q] it is also simple as an ungraded ring. Also, as will be used frequently in the body of the paper,

the only graded right or left ideals of $T_{(g)}$ are the $g^{n} T_{(g)}$.

For any graded vector subspace $X \subseteq T_{(g)}$, set

$$
\widehat{X}=\left\{t \in T_{(g)}: \operatorname{tg}^{n} \in X \text { for some } n \in \mathbb{N}\right\} .
$$

We say that $X$ is $g$-divisible if $X \cap g T_{(g)}=g X$. Note that if $X$ is $g$-divisible and $1 \in X$ (as happens when $X$ is a subring of $T_{(g)}$ ), then $g \in X$. For any $\mathbb{k}$-subspace $Y$ of $T_{(g)}$, write $\bar{Y}=\left(Y+g T_{(g)}\right) / g T_{(g)}$ for the image of $Y$ in $T_{(g)} / g T_{(g)}$.

If $R \subseteq T_{(g)}$ is a subalgebra with $g \in R$, then the $g$-torsion submodule of a right $R$-module $M$ is $\operatorname{tors}_{g}(M)=\left\{m \in M: g^{n} m=0\right.$ for some $\left.n \geq 1\right\}$. We say that $M$ is $g$-torsionfree if tors $g(M)=0$ and $g$-torsion if $\operatorname{tors}_{g}(M)=M$.

We notice that the rings $T$ automatically satisfy some useful additional properties. An algebra $C$ is called just infinite if every nonzero ideal $I$ of $C$ satisfies $\operatorname{dim}_{\mathbb{k}} C / I<\infty$.

Lemma 2.7. Let $T$ satisfy Assumption 2.1. Then:

(1) $T$ is generated as an algebra in degree 1.

(2) Any finitely generated, cg subalgebra of $Q_{\mathrm{gr}}(T / g T)=\mathbb{k}(E)\left[z, z^{-1} ; \tau\right]$, in particular $T / g T$ itself, is just infinite.

Proof. (1) Since $\mu \geq 2$, the ring $B=T / g T \cong B(E, M, \tau)$ is generated in degree 1 [Rogalski 2011, Lemma 3.1]. Thus $T_{2}=\left(T_{1}\right)^{2}+g T_{1}=\left(T_{1}\right)^{2}$ and, by induction, $\left(T_{1}\right)^{n}=T_{n}$ for all $n \geq 1$.

(2) This follows from [RSS 2014, Corollary 2.10 and §3].

As the next few results show, $g$-divisible algebras and modules have pleasant properties. The first gives a useful, albeit easy, alternative characterisation of $\widehat{X}$ that will be used without particular reference.

Lemma 2.8. Let $R \subseteq T_{(g)}$ be a cg subalgebra with $g \in R$, and let $X \subseteq T_{(g)}$ be a graded right $R$-module. Then $X \subseteq \widehat{X}$, and $\widehat{X}$ is also a right $R$-module. Moreover:

$X$ is g-divisible $\Longleftrightarrow X=\widehat{X} \Longleftrightarrow T_{(g)} / X$ is a g-torsionfree $R$-module. 
Proposition 2.9. (1) If $R$ is any $g$-divisible cg subalgebra of $T$, then $R$ is finitely generated as a $\mathbb{k}$-algebra.

(2) Let $R$ be a finitely generated $g$-divisible cg subalgebra of $T_{(g)}$. Then $R$ is strongly noetherian.

Proof. (1) We have $\bar{R} \cong(R+g T) / g T \subseteq \bar{T} \cong B(E, M, \tau)$ and so [RSS 2014, Theorem 2.9] implies that $\bar{R}$ is noetherian. By [Artin et al. 1990, Lemma 8.2], $R$ is noetherian. Since the generators of $R_{\geq 1}$ as an $R$-module also generate $R$ as a k-algebra, $R$ is finitely generated as a $\mathbb{k}$-algebra.

(2) In this case, $\bar{R}=R / g R \cong\left(R+g T_{(g)}\right) / g T_{(g)} \subseteq Q_{\mathrm{gr}}(B)=\mathbb{k}(E)\left[t, t^{-1} ; \tau\right]$. By [RSS 2014, Corollary 2.10] $\bar{R}$ is noetherian. Also GKdim $\bar{R} \leq 2$, for instance by [Artin and Stafford 1995, Theorem 0.1], and so $\bar{R}$ is strongly noetherian by [Artin et al. 1999, Theorem 4.24]. Thus $R$ is strongly noetherian by [Artin et al. 1990, Lemma 8.2].

Lemma 2.10. Let $R$ be a g-divisible cg subalgebra of $T_{(g)}$ with $D_{\mathrm{gr}}(R)=D_{\mathrm{gr}}(T)$. Then

(1) $Q_{\mathrm{gr}}(R)=Q_{\mathrm{gr}}(T)$, and

(2) $Q_{\mathrm{gr}}(\bar{R})=Q_{\mathrm{gr}}(\bar{T})$.

Proof. (1) As $g \in R_{1}$ we have

$$
Q_{\mathrm{gr}}(T)=D_{\mathrm{gr}}(T)\left[g, g^{-1}\right]=D_{\mathrm{gr}}(R)\left[g, g^{-1}\right]=Q_{\mathrm{gr}}(R) .
$$

(2) Since $Q_{\mathrm{gr}}(R)=Q_{\mathrm{gr}}(T)$, there exists $0 \neq x \in R_{d}$ such that $x T_{1} \subseteq R_{d+1}$. Then $\bar{x} \bar{T}_{1} \subseteq \bar{R}$. As long as $\bar{x} \neq 0$, this shows that the graded quotient ring of $\bar{R}$ contains a generating set for $\bar{T}$ and we are done. On the other hand, if $\bar{x}=0$, then write $x=g^{i} y$ with $y \in T_{(g)} \backslash g T_{(g)}$; equivalently $y \in R \backslash g R$ by $g$-divisibility. Then $g^{i} y T_{1} \subseteq R \cap g^{i} T_{(g)}=g^{i} R$, and so $y T_{1} \subseteq R$. Thus we are again done.

If $A$ is a cg domain with graded quotient ring $Q=Q_{\mathrm{gr}}(A)$ and $M \subseteq Q$ is a finitely generated graded right $A$-submodule, we can and always will identify

$$
\begin{aligned}
\operatorname{End}_{A}(M) & =\{q \in Q: q M \subseteq M\} \quad \text { and } \\
M^{*} & =\operatorname{Hom}_{A}(M, A)=\{q \in Q: q M \subseteq A\} .
\end{aligned}
$$

Clearly both $\operatorname{End}_{A}(M)$ and $M^{*}$ are graded subspaces of $Q$.

Lemma 2.12. Let $R$ be any $g$-divisible subring of $T_{(g)}$ with $Q_{\mathrm{gr}}(R)=Q_{\mathrm{gr}}\left(T_{(g)}\right)$, and let $M, M^{\prime} \subseteq T_{(g)}$ be finitely generated nonzero right $R$-modules.

(1) If $M \nsubseteq g T_{(g)}$, then we can identify

$$
\operatorname{Hom}_{R}\left(M, M^{\prime}\right)=\left\{x \in T_{(g)}: x M \subseteq M^{\prime}\right\} \subseteq T_{(g)} .
$$


(2) If $M^{\prime}$ is $g$-divisible, and $M \nsubseteq g T_{(g)}$ (in particular if $M$ is $g$-divisible) then $\operatorname{Hom}_{R}\left(M, M^{\prime}\right) \subseteq T_{(g)}$ is also g-divisible.

(3) If $M$ is $g$-divisible, then $U=\operatorname{End}_{R}(M) \subseteq T_{(g)}$ is $g$-divisible, and $M$ is a finitely generated left $U$-module. Moreover, $\bar{U} \subseteq \operatorname{End}_{\bar{R}}(\bar{M})$.

Proof. (1) Since $M \nsubseteq g T_{(g)}$, it follows from (2.6) that $M T_{(g)}=T_{(g)}$. In particular,

$$
N=\operatorname{Hom}_{R}\left(M, M^{\prime}\right) \subseteq \operatorname{Hom}_{T_{(g)}}\left(M T_{(g)}, M^{\prime} T_{(g)}\right) \subseteq T_{(g)} .
$$

(2) Part (1) applies, and so $N=\operatorname{Hom}_{R}\left(M, M^{\prime}\right) \subseteq T_{(g)}$. Next, let $\theta \in N \cap g T_{(g)}$; say $\theta=g s$ for some $s \in T_{(g)}$. Then $s g M=\theta M \in M^{\prime} \cap g T_{(g)}=M^{\prime} g$ since $M^{\prime}$ is $g$-divisible. Hence $s M \subseteq M^{\prime}$ and $s \in N$. Thus $N \cap g T_{(g)}=g N$.

(3) By part (2), $U$ is $g$-divisible, and hence is noetherian by Proposition 2.9. As $Q_{\mathrm{gr}}(R)=Q_{\mathrm{gr}}\left(T_{(g)}\right)$, there exists $x \in T_{(g)} \backslash\{0\}$ so that $x M \subseteq R$. Then $M x M \subseteq$ $M R=M$. Hence (up to a shift) $M \cong M x \subseteq U$ is finitely generated as a left $U$-module.

Now

$$
\bar{U}=\left(U+g T_{(g)}\right) / g T_{(g)} \subseteq \overline{T_{(g)}}=\mathbb{k}(E)\left[t, t^{-1} ; \tau\right] .
$$

Since $Q_{\mathrm{gr}}(\bar{U})=Q_{\mathrm{gr}}\left(\overline{T_{(g)}}\right)$ by Lemma 2.10, as in (2.11) we identify $\operatorname{End}_{\bar{R}}(\bar{M})$ with $\left\{x \in \overline{T_{(g)}}: x \bar{M} \subseteq \bar{M}\right\}$. But since $U M \subseteq M$, clearly $(\bar{U})(\bar{M}) \subseteq \bar{M}$.

Lemma 2.13. Let $R$ be a graded subalgebra of $T_{(g)}$ with $Q_{\mathrm{gr}}(R)=Q_{\mathrm{gr}}\left(T_{(g)}\right)$ and let $M \subseteq T_{(g)}$ be a graded right $R$-submodule of $T_{(g)}$ such that $M \nsubseteq g T_{(g)}$. Then:

(1) For any $x \in T_{(g)} \backslash g T_{(g)}$, we have $\widehat{x M}=x \widehat{M}$.

(2) If $R$ is $g$-divisible and $M$ is a finitely generated $R$-module, then so is $\widehat{M}$.

(3) If $R$ is $g$-divisible, then $T_{(g)} \supseteq M^{*}=\widehat{M^{*}}$ and $M^{*} \nsubseteq g T_{(g)}$. Hence $T_{(g)} \supseteq M^{* *}=$ $\widehat{M^{* *}}$. Moreover, we have $(\widehat{M})^{*}=M^{*}$ and $(\widehat{M})^{* *}=M^{* *}$.

Proof. (1) Let $r \in \widehat{M}$. For some $n$ we have $r g^{n} \in M$, so $x r g^{n} \in x M$. Since $x r \in T_{(g)}$ it follows that $x r \in \widehat{x M}$. Conversely, if $r \in T_{(g)}$ with $r g^{n} \in x M$, then $r g^{n}=g^{n} r \in g^{n} T_{(g)} \cap x T_{(g)}$. As $g T_{(g)}$ is a completely prime ideal and $x \notin g T_{(g)}$, clearly $g^{n} T_{(g)} \cap x T_{(g)}=g^{n} x T_{(g)}$. Thus $r=x s$ for some $s \in T_{(g)}$ and $x M \ni r g^{n}=x s g^{n}$. Therefore $s g^{n} \in M$, whence $s \in \widehat{M}$ and $r \in x \widehat{M}$. Thus $\widehat{x M}=x \widehat{M}$, as claimed.

(2) As in the proof of Lemma 2.12, there exists $x \in T_{(g)} \backslash\{0\}$ so that $x M \subseteq R$. If $x=g y$ for some $y \in T_{(g)}$, then $g(y M) \subseteq R$ and so $y M \subseteq R$ since $R$ is $g$-divisible. Thus we can assume that $x \in T_{(g)} \backslash g T_{(g)}$. Again by $g$-divisibility, $\widehat{x M} \subseteq \widehat{R}=R$. By Proposition $2.9 \widehat{x M}$ is a finitely generated right ideal of $R$. Up to a shift, $\widehat{M} \cong x \widehat{M}=\widehat{x M}$ by (1). This is finitely generated as an $R$-module.

(3) By Lemma 2.12(2), $M^{*}$ is equal to $\operatorname{Hom}_{R}(M, R) \subseteq T_{(g)}$ and is $g$-divisible, i.e., $M^{*}=\widehat{M^{*}}$. Clearly then $M^{*} \nsubseteq g T_{(g)}$, and so by the left-handed analogue of the same argument, $M^{* *}=\widehat{M^{* *}} \subseteq T_{(g)}$ also. 
Now as $M \subseteq \widehat{M}$, certainly $(\widehat{M})^{*} \subseteq M^{*}$. On the other hand, if $\theta \in M^{*}$ and $x \in \widehat{M}$, say with $x g^{n} \in M$, then $(\theta x) g^{n}=\theta\left(x g^{n}\right) \in R=\widehat{R}$. Hence $\theta x \in R$. Thus $\theta \in(\widehat{M})^{*}$ and $(\widehat{M})^{*}=M^{*}$. Taking a second dual gives $(\widehat{M})^{* *}=M^{* *}$.

We note next some special properties of modules of GK-dimension 1.

Lemma 2.14. Let $R$ be a cg $g$-divisible subalgebra of $T_{(g)}$ and suppose that $M$ is a finitely generated, $g$-torsionfree $R$-module with $\operatorname{GKdim}(M) \leq 1$. Then the Hilbert series of $M$ is eventually constant; that is, $\operatorname{dim}_{\mathbb{k}} M_{n}=\operatorname{dim}_{\mathbb{k}} M_{n+1}$ for all $n \gg 0$. Moreover, $M$ is a finitely generated $\mathbb{k}[\mathrm{g}]$-module.

Proof. By [Krause and Lenagan 1985, Proposition 5.1(e)], GKdim $(M / M g) \leq 0$ and so $\operatorname{dim}_{\mathbb{k}} M / M g<\infty$. Thus $M_{r} g=M_{r+1}$ for all $r \gg 0$; say for $r \geq n_{0}$. In particular, $M=M_{\leq n_{0}} \mathbb{k}[g]$. Moreover, since multiplication by $g$ is an injective map from $M_{r}$ to $M_{r+1}$, it follows that $\operatorname{dim}_{\mathbb{k}} M_{r}=\operatorname{dim}_{\mathbb{k}} M_{r+1}$ for all $r \geq n_{0}$.

A graded ideal $I$ in a cg algebra $R$ is called a sporadic ideal if $\operatorname{GKdim}(R / I)=1$ (these are called special ideals in [Rogalski 2011]). The name is justified since, as will be shown in Section 8, orders in $T$ have very few such ideals. The next lemma will be useful in understanding them.

Lemma 2.15. Let $R$ be a g-divisible finitely generated cg subring of $T_{(g)}$ with $Q_{\mathrm{gr}}(R)=Q_{\mathrm{gr}}(T)$. Then:

(1) If $J$ is a nonzero $g$-divisible graded ideal of $R$, then $\operatorname{GKdim}(R / J) \leq 1$.

(2) Conversely, if $J$ is a graded ideal of $R$ such that $\operatorname{GKdim}(R / J) \leq 1$, then $\widehat{J} / J$ is finite-dimensional.

(3) If $K$ is any ideal of $R$, then $K=g^{n} I$ for some $n \geq 1$ and ideal I satisfying $\operatorname{GKdim}(R / I) \leq 1$.

(4) Suppose that $L, M$ are graded subspaces of $T_{(g)}$ with $L \nsubseteq g T_{(g)}$ and $M \nsubseteq g T_{(g)}$ and assume that $I=L M$ is an ideal of $R$. Then $\operatorname{GKdim}(R / I) \leq 1$.

Proof. (1) By Lemma 2.10, $\bar{R} \subseteq k(E)\left[t, t^{-1} ; \tau\right]=Q_{\mathrm{gr}}(\bar{R})$ and, by Lemma 2.7(2), $\bar{R}$ is just infinite. Since $J$ is $g$-divisible, $J \nsubseteq g R$ and so $\bar{J} \neq 0$; thus $\operatorname{dim}_{\mathbb{k}} \bar{R} / \bar{J}<\infty$. Equivalently, if $R^{\prime}=R / J$ then $\operatorname{dim}_{\mathbb{k}} R^{\prime} / g R^{\prime}<\infty$. It follows that $R_{m}^{\prime}=g R_{m-1}^{\prime}$ for all $m \gg 0$, and hence that GKdim $R^{\prime} \leq 1$.

(2) Once again, $\bar{R}$ is just infinite. Thus, since $J \subseteq g R$ would lead to the contradiction $\operatorname{GKdim}(R / J) \geq 2$, we must have $\operatorname{dim}_{\mathbb{k}} R /(g R+J)=\operatorname{dim}_{\mathbb{k}} \bar{R} / \bar{J}<\infty$. Since $\widehat{J}$ is noetherian, $g^{n} \widehat{J} \subseteq J$ for some $n$. If $J^{\prime}$ is the largest right ideal inside $\widehat{J}$ such that $J^{\prime} / J$ is finite-dimensional, then $J^{\prime}$ is an ideal and we can replace $J$ by $J^{\prime}$ without loss. If we still have $J \neq \widehat{J}$, then there exists $x \in \widehat{J} \backslash J$ such that $x g \in J$. Thus $x(g R+J) \subseteq J$, and left multiplication by $x$ defines a surjection $R /(g R+J) \rightarrow(x R+J) / J$. We have $\operatorname{dim}_{\mathbb{k}}(x R+J) / J=\infty$ and $\operatorname{dim}_{\mathbb{k}} R /(g R+J)<\infty$, a contradiction. Thus $\widehat{J}=J$. 
(3) Write $K=g^{n} J$ with $n$ as large as possible and $J$ an ideal of $R$. Then $J \nsubseteq g R$, and so Lemma 2.7(2) again implies that $\operatorname{dim}_{\mathbb{k}} \bar{R} / \bar{J}<\infty$ and hence GKdim $R / J \leq 1$.

(4) Since $g T_{(g)}$ is completely prime, $I=L M \nsubseteq g T_{(g)}$ and hence $I \nsubseteq g R$. Now apply part (3).

Next, we want to prove some general results about equivalent orders that will be useful elsewhere. We recall that two cg domains $A$ and $B$ with a common (graded) quotient ring $Q=Q_{\mathrm{gr}}(A)=Q_{\mathrm{gr}}(B)$ are equivalent orders if $a A b \subseteq B$ and $c B d \subseteq A$ for some $a, b, c, d \in Q \backslash\{0\}$. Clearing denominators on the appropriate sides, one can always assume that $a, b, c, d \in B$. One can also assume that $a, b, c, d$ are homogeneous; indeed, if $a$ and $b$ have leading terms $a_{n}$ and $b_{m}$, then $a_{n} A b_{m} \subseteq B$.

Proposition 2.16. Suppose that $U \subseteq R$ are g-divisible cg finitely generated subalgebras of $T_{(g)}$ such that $Q_{\mathrm{gr}}(U)=Q_{\mathrm{gr}}(R)=Q_{\mathrm{gr}}\left(T_{(g)}\right)$. Then the following are equivalent:

(1) $U$ and $R$ are equivalent orders in $Q_{\mathrm{gr}}(U)=Q_{\mathrm{gr}}(T)$.

(2) $U / g U$ and $R / g R$ are equivalent orders in $Q_{\mathrm{gr}}(U / g U)$.

Proof. (1) $\Rightarrow$ (2) Choose nonzero homogeneous elements $a, b \in U$ such that $a R b \subseteq U$. Write $a=g^{n} a^{\prime}$ where $a^{\prime} \in U \backslash g U$. Then $g^{n} a^{\prime} R b \subseteq U$ and so $a^{\prime} R b \subseteq U$ since $U$ is $g$-divisible. Replacing $a$ by $a^{\prime}$, we can assume that $a \notin g U$ and, similarly, that $b \notin g U$. Then $\bar{a} \bar{R} \bar{b} \subseteq \bar{U}$, with $\bar{a}, \bar{b} \neq 0$, as required.

(2) $\Rightarrow$ (1) Set $\bar{U}=U / g U \subseteq \bar{R}=R / g R$. We first note that there is a subalgebra $\bar{U} \subseteq S \subseteq \bar{R}$ so that $S$ is a noetherian right $\bar{U}$-module and $\bar{R}$ is a noetherian left $S$-module. Indeed, write $a \bar{R} b \subseteq \bar{U}$ for some nonzero $a, b \in \bar{U}$ and set $S=\bar{U}+\bar{R} b \bar{U}$. Clearly $a S \subseteq \bar{U}$ and $\bar{R} b \subseteq S$. As in the proof of Proposition 2.9, all subalgebras of $\bar{R}$ are noetherian. In particular, $S$ and $\bar{U}$ are noetherian and so these inclusions ensure that $S_{\bar{U}}$ and ${ }_{S} \bar{R}$ are finitely generated, as claimed.

Let $F \subseteq R$ be a finite-dimensional vector space, containing 1 , such that $\bar{F} \bar{U}=S$. Set $M=\widehat{F U}$ and $V=\operatorname{End}_{U}(M)$. Clearly $Q_{\mathrm{gr}}(V)=Q_{\mathrm{gr}}(U)=Q_{\mathrm{gr}}(T)$. Since $1 \in M$ and hence $M \nsubseteq g T_{(g)}$, we can and will use Lemma 2.12(1) to identify $V=\left\{q \in Q_{\text {gr }}(U): q M \subseteq M\right\} \subseteq T_{(g)}$. By Lemma 2.12(3), $V=\widehat{V}$ and ${ }_{V} M$ is finitely generated, while, by Lemma $2.13, M_{U}$ is finitely generated. As $R$ is $g$-divisible and $F U \subseteq R$, we have $M \subseteq R$. Since $1 \in M$ this implies that $M R=R$. Hence $V R=V M R=M R=R$ and $V \subseteq R$.

Let $G, H \subseteq R$ be finite-dimensional vector spaces with $V G=M$ and $S \bar{H}=\bar{R}$. Then

$$
\bar{R} \supseteq \overline{V G H} \supseteq \overline{F U H}=S \bar{H}=\bar{R} .
$$

Thus $\bar{R}=\overline{M H}=\overline{V G H}$ is finitely generated as a left $\bar{V}$-module. Since $g \in V_{+}=$ $\bigoplus_{i>0} V_{i} \subseteq R_{+}$, this implies that $R /\left(V_{+}\right) R$ is a finitely generated left module over 
$V / V_{+}$. By the graded analogue of Nakayama's lemma, this implies that $R$ is finitely generated as a left $V$-module. Thus $R$ and $V$ are equivalent orders. As $V$ and $U$ are equivalent orders (via the bimodule $M$ ), it follows that $U$ and $R$ are equivalent.

\section{Curves}

The main result of [Artin and Stafford 1995] shows that any cg domain A of GelfandKirillov dimension two has a Veronese ring that is an idealiser inside a TCR. In this section we strengthen this result for elliptic curves by proving that, for subalgebras of a TCR over such a curve corresponding to an automorphism of infinite order, the result holds without taking a Veronese ring, although at the cost of replacing the idealiser by an algebra which is isomorphic to an idealiser in large degree.

Given graded modules $M, N \subseteq P$ over a cg algebra $A$, we write $M \doteq N$ if $M$ and $N$ agree up to a finite-dimensional vector space. If $M, N \in \operatorname{gr}-A$, this is equivalent to $M_{\geq n}=N_{\geq n}$ for some $n \geq 0$.

Theorem 3.1. Let $A$ be a cg ring such that $Q_{\mathrm{gr}}(A)=\mathbb{k}(E)\left[z, z^{-1} ; \tau\right]$ for some infinite-order automorphism $\tau$ of a smooth elliptic curve $E$ and $z \in Q_{\mathrm{gr}}(A)_{1}$. Then there are an ideal sheaf $\mathscr{A}$ and an ample invertible sheaf $\mathscr{H}$ on $E$ so that

$$
A \doteq \bigoplus_{n \geq 0} H^{0}\left(E, A_{\mathcal{H}_{n}}\right)
$$

Remarks 3.2. (1) The idealiser $\llbracket(J)=\rrbracket_{U}(J)$ of a right ideal $J$ in a ring $U$ is the subring

$$
\llbracket(J)=\{u \in U: u J \subseteq J\} .
$$

In the notation of the theorem, $J=\bigoplus_{n \geq 0} H^{0}\left(E, \mathscr{A}_{\mathcal{H}_{n}}\right)$ is a right ideal of the TCR $B(E, \mathcal{H}, \tau)$; further, $\rrbracket_{U}(J) \doteq \mathbb{}$ $+J$. So, an equivalent way of phrasing the theorem is to assert that (up to a finite-dimensional vector space) $A$ is equal to the idealiser $\llbracket(J)$ inside $B(E, \mathscr{H}, \tau)$.

(2) The assertion that $z \in Q_{\mathrm{gr}}(A)_{1}$ can be avoided at the expense of regrading $A$, although in the process one must replace $\tau$ by some $\tau^{m}$ in the definition of the $\mathscr{H}_{n}$.

(3) The sheaf $\mathscr{H}$ is ample if and only if it has positive degree [Hartshorne 1977, Corollary 3.3], if and only if $\mathscr{H}$ is $\tau$-ample: that is, for any coherent $\mathscr{F}$ and for $n \gg 0$, the sheaf $\mathscr{F}_{\mathcal{E}} \otimes \mathscr{H}_{n}$ is globally generated with $H^{1}\left(E, \mathscr{F}_{\mathcal{H}} \otimes \mathscr{H}_{n}\right)=0$ [Artin and Van den Bergh 1990, Corollary 1.6].

Proof. The hypothesis on $z$ ensures that $A_{p} \neq 0 \neq A_{p+1}$ for all $p \gg 0$. Fix some such $p$.

The conclusion of the theorem is, essentially, the same as that of [Artin and Stafford 1995, Theorem 5.11], although that result has two hypotheses we need to remove. The first, [ibid., Hypothesis 2.1] requires that the ring in question 
has a nonzero element in degree one, so does at least hold for the Veronese rings $A^{(p)}$ and $A^{(q)}$, for $q=p+1$. The remaining hypothesis, [ibid., Hypothesis 2.15], concerns $\tau$-fixed points of $E$. In our situation, this automatically holds as $E$ has no such fixed points (see the discussion before [ibid., (2.9)]).

By the discussion above, [ibid., Theorem 5.11 and Remark 5.12(2)] can be applied to the Veronese rings $A^{(p)}$ and $A^{(q)}$. This provides invertible sheaves $\mathscr{A}, \mathscr{B}, \mathscr{F}, \mathscr{G}$ with $\mathscr{F}, \mathscr{G}$ ample such that

$$
A^{(p)} \doteq \bigoplus_{n \geq 0} H^{0}\left(E, \mathscr{A} \otimes \mathscr{F}_{p, n}\right) \quad \text { and } \quad A^{(q)} \doteq \bigoplus_{n \geq 0} H^{0}\left(E, \mathscr{B} \otimes \mathscr{G}_{q, n}\right),
$$

where in order to take account of the Veronese rings we have written $\mu_{r, n}=$ $\mathcal{M} \otimes \mathcal{M}^{\tau^{r}} \otimes \cdots \otimes \mathcal{M}^{\tau^{(n-1) r}}$ for an invertible sheaf $\mathcal{M}$. For $n \gg 0$ the sheaves $\mathscr{A} \otimes \mathscr{F}_{p, n q}$ and $\mathscr{B} \otimes \mathscr{G}_{q, n p}$ are generated by their sections $A_{n p q}$ and so $\mathscr{A} \otimes \mathscr{F}_{p, n q}=\mathscr{B} \otimes \mathscr{G}_{q, n p}$ for such $n$. Replacing $n$ by $n+m$, we obtain

$$
\begin{aligned}
\mathscr{A} \otimes \mathscr{F}_{p, n q} \otimes \mathscr{F}_{p, m q}^{\tau^{n p q}} & =\mathscr{A} \otimes \mathscr{F}_{p,(n+m) q}=\mathscr{B} \otimes \mathscr{G}_{q,(n+m) p} \\
& =\mathscr{B} \otimes \mathscr{G}_{q, n p} \otimes \mathscr{G}_{q, m p}^{\tau^{n p q}}=\mathscr{A} \otimes \mathscr{F}_{p, n q} \otimes \mathscr{G}_{q, m p}^{\tau^{n p q}}
\end{aligned}
$$

for all $n+m>n \gg 0$. Cancelling the first two terms and applying $\tau^{-n p q}$ gives $\mathscr{F}_{p, m q}=\mathscr{G}_{q, m p}$ for all $m \geq 1$. In particular, it holds for $m=n$, and hence $\mathscr{A}=\mathscr{B}$.

Next, set $\mathscr{H}=\mathscr{G} \otimes\left(\mathscr{F}^{\tau}\right)^{-1}$; thus the equation $\mathscr{F}_{p, q}=\mathscr{G}_{q, p}$ gives

$$
\mathscr{H}_{q, p}=\mathscr{F}_{p, q} \otimes\left(\mathscr{F}^{\tau}\right)_{q, p}^{-1} .
$$

We claim that $\mathscr{F}_{F}$ is the unique invertible sheaf $\widetilde{\mathscr{F}}_{\text {satisfying }} \mathscr{H}_{q, p}=\widetilde{\mathscr{F}}_{p, q} \otimes\left(\widetilde{\mathscr{F}}^{\tau}\right)_{q, p}^{-1}$. To see this, suppose that $\widetilde{\mathscr{F}}$ is a second sheaf satisfying this property and consider associated divisors. Pick a closed point $x \in E$ and write $\mathbb{O}_{x}=\left\{x(i)=\tau^{-i}(x): i \in \mathbb{Z}\right\}$ for the orbit of $x$ under $\tau$. Writing $\mathscr{F}=O_{E}(F)$ and $\mathscr{H}=\mathscr{O}_{E}(H)$ for some divisors $F$ and $H$ and restricting to $\mathbb{O}=\mathbb{O}_{x}$ gives $\left.F\right|_{\mathbb{O}}=\sum m(i) x(i)$ and $\left.H\right|_{\mathbb{O}}=\sum r(i) x(i)$, for some integers $m(i), r(i)$ (the notation is chosen to avoid excessive subscripts). Now, in terms of divisors, (3.3) gives

$$
\begin{aligned}
& \sum r(i) x(i)+r(i) x(i+q)+\cdots+r(i) x(i+(p-1) q) \\
& =\sum m(i) x(i)+m(i) x(i+p)+\cdots+m(i) x(i+(q-1) p) \\
& \quad-\sum m(i) x(i+1)+m(i) x(i+1+q)+\cdots+m(i) x(i+1+(p-1) q) .
\end{aligned}
$$

Equating coefficients of $x(t)$ in the last displayed equation gives

$$
\begin{aligned}
m(t)+m(t-p)+\cdots+m(t-(q-1) p) & \\
-m(t-1)-m(t-1-q) & -\cdots-m(t-1-(p-1) q) \\
& =r(t)+r(t-q)+\cdots+r(t-(p-1) q) .
\end{aligned}
$$


Recall that $m(i)=r(i)=0$ for $|i| \gg 0$. Therefore, solving this system from $t \ll 0$ through to $t \gg 0$ gives a unique solution for the $m(i)$ in terms of the $r(j)$. Finally, doing this for every orbit involved in the divisors $F$ and $H$ shows that $F$ is uniquely determined by $H$, and so $\mathscr{F}$ is uniquely determined as claimed.

A direct calculation shows that if $\widetilde{\mathscr{F}}=\mathscr{H}_{1, p}=\mathscr{H}_{p}$ then $\mathscr{H}_{q, p}=\widetilde{\mathscr{F}}_{p, q} \otimes\left(\widetilde{\mathscr{F}}^{\tau}\right)_{q, p}^{-1}$. Thus $\widetilde{\mathscr{F}}=\mathscr{F}_{F}$ and consequently $\mathscr{H}_{1, m p}=\mathscr{F}_{p, m}$ for all $m \geq 1$. It follows from the equation $\mathscr{H}=\mathscr{G} \otimes\left(\mathscr{F}^{\tau}\right)^{-1}$ that $\mathscr{G}_{=} \mathscr{H}_{1, q}$, and thus $\mathscr{H}_{1, m q}=\mathscr{G}_{q, m}$ for all $m \geq 1$ as well. To summarise, we have found sheaves $\mathscr{A}$ and $\mathscr{H}$ such that

$$
A^{(s)} \doteq \bigoplus_{n} H^{0}\left(E, \mathscr{A H}_{1, n s}\right)=\bigoplus_{n} H^{0}\left(E, \mathscr{A H}_{n s}\right) \quad \text { for } s=p, p+1 .
$$

It follows that (3.4) holds for all $s \gg 0$, but this is not quite enough to prove the theorem since, as $s$ increases, one has no control over the finitely many values of $n=n(s)$ for which $A_{n}^{(s)} \neq H^{0}\left(E, A_{\mathcal{H}_{1, n s}}\right)$. So we take a slightly different tack.

For $0 \leq r \leq p-1$, write $M(r)=\bigoplus_{n \geq 0} A_{n p+r}$; thus $A=\bigoplus_{r=0}^{p-1} M(r)$. Fix some such $r$. We can find $0 \neq x \in A_{2 p-r}$, since $2 p-r>p$. Thus $x M(r) \subseteq A^{(p)}$ and so, by [ibid., Proposition 5.4] and (3.4), there exists an ideal sheaf $\mathscr{I} \subseteq \mathbb{O}_{E}$ such that $x M(r) \doteq \bigoplus_{n \geq 0} H^{0}\left(E, \mathscr{I} \otimes \mathcal{H}_{1, n p}^{\tau^{2 p}}\right)$ (in this formula, the twist by $\tau^{2 p}$ is for convenience only but it will simplify the computations). Since $A$ is a domain, $M(r)$ is isomorphic to the shift $x M(r)[2 p-r]$. Hence, for some integer $n_{0}$ independent of $r$, [Keeler et al. 2005, Lemma 5.5] implies that

$$
M(r)_{\geq n_{0}}=\bigoplus_{n \geq n_{0}} H^{0}\left(E, \mathscr{I}^{\prime} \otimes \mathcal{H}_{1, n p}^{\tau^{r}}\right)=\bigoplus_{n \geq n_{0}} H^{0}\left(E, \mathscr{g}(r) \otimes \mathcal{H}_{r} \otimes \mathcal{H}_{1, n p}^{\tau^{r}}\right)
$$

for some invertible sheaves $\mathscr{I}^{\prime}$ and $\mathscr{f}(r)=\mathscr{I}^{\prime} \otimes\left(\mathscr{H}_{r}\right)^{-1}$. Possibly after increasing $n_{0}$, we may also assume that the sheaves in (3.4) and (3.5) are generated by their sections for $n \geq n_{0}$. Now pick $n \geq n_{0}$ such that $r+n p=(p+1) m$ for some $m$. Then comparing (3.4) and (3.5) shows that $M(r)_{r+n p}$ generates the sheaves

$$
\mathscr{F}(r) \otimes \mathscr{H}_{r+n p}=\mathscr{F}(r) \otimes \mathscr{H}_{r} \otimes \mathscr{H}_{1, n p}^{\tau^{r}}=\mathscr{A} \otimes \mathscr{H}_{1,(p+1) m}=\mathscr{A} \otimes \mathscr{H}_{r+n p} .
$$

Hence $\mathscr{f}(r)=\mathscr{A}$. Since this holds for all $0 \leq r \leq p-1$, it follows that $A_{n}=$ $H^{0}\left(E, \mathscr{A}^{\tau^{n}}\right)$, for all $n \geq n_{0} p$.

Remark 3.6. We note that [Rogalski 2011, Lemma 3.2(2)] states a result similar to Theorem 3.1, but the proof erroneously quotes the relevant theorems from [Artin and Stafford 1995] without removing the hypothesis that rings should have a nonzero element in degree one. Thus the above proof also corrects this oversight. In any case, [Rogalski 2011, Lemma 3.2(2)] was only used in that paper for rings generated in degree one.

If $A$ is a cg algebra generated in degree one, then we define a point module to be a cyclic module $M=\bigoplus_{i \geq 0} M_{i}$, with $\operatorname{dim} M_{i}=1$ for all $i \geq 0$. When $A$ is 
not generated in degree one, a point module has this asymptotic structure, but the precise definition can vary depending on circumstances, and so we will be careful to explain which definition we mean should the distinction be important.

To end this section we give some applications of the previous theorem to the structure of point modules, for which we need a definition. If $M=\bigoplus_{n} M_{n}$ is a graded module over a cg algebra $A$, we write $s^{n}(M)=\left(M_{n} A\right)[n]$. The largest artinian submodule of a noetherian module $M$ is written $S(M)$.

Corollary 3.7. Let A satisfy the hypotheses of Theorem 3.1. Let $M$ and $M^{\prime}$ be 1-critical graded right A-modules generated in degree zero. Then:

(1) the isomorphism classes of such modules are in one-to-one correspondence with the closed points of $E$;

(2) $\operatorname{dim} M_{n} \leq 1$ for all $n \geq 0$, with $\operatorname{dim} M_{n}=1$ for $n \gg 0$;

(3) for $n \geq 0$, either $M_{n}=0$ or $s^{n} M$ is cyclic and 1-critical;

(4) if $s^{n} M \cong s^{n} M^{\prime} \neq 0$ for some $n \in \mathbb{N}$, then $M \cong M^{\prime}$.

Proof. It is well known that there is an equivalence of categories qgr- $A \sim \operatorname{coh}(E)$, and much of the corollary follows from this; thus we first review the details of the equivalence. By [Artin and Stafford 1995, Theorem 5.11] and the left-right analogue of Theorem 3.1, we can write

$$
A \doteq \bigoplus_{n \geq 0} H^{0}\left(E, \mathscr{H}_{n} \mathscr{A}^{\tau^{n-1}}\right) \subseteq B=B(E, \mathscr{H}, \tau)
$$

for some ideal sheaf $\mathscr{A}$ and invertible sheaf $\mathscr{H}$. For $n_{0} \gg 0$, the ideal

$$
J=A_{\geq n_{0}}=\bigoplus_{n \geq n_{0}} H^{0}\left(E, \mathscr{H}_{n} \mathscr{A}^{\tau^{n-1}}\right)
$$

is a left ideal of $B$. By [Stafford and Zhang 1994, Proposition 2.7] and its proof, qgr- $A \sim$ qgr- $B$ under the maps $\alpha: N \mapsto N \otimes_{A} B$ and $\beta: N^{\prime} \mapsto N^{\prime} \otimes_{B} J$. Moreover, by [Artin and Van den Bergh 1990, Theorem 1.3], qgr- $B \sim \operatorname{coh}(E)$. Under that equivalence, for a closed point $p$ of $E$ the skyscraper sheaf $\mathbb{k}(p) \in \operatorname{coh}(E)$ maps to the module

$$
M_{p}^{\prime}=\bigoplus_{n \geq 0} H^{0}\left(E, k(p) \otimes \mathscr{H}_{n}\right) \in \mathrm{qgr}-B
$$

thus if $M_{p}=M_{p}^{\prime} / S\left(M_{p}^{\prime}\right)$ then $M_{p}$ is a 1-critical $B$-module with $\operatorname{dim}\left(M_{p}\right)_{n}=1$ for $n \gg 0$. By [Stafford and Zhang 1994, Lemma 2.6] the same is true of the 1-critical $A$-module $N_{p}=\beta\left(M_{p}\right) / S\left(\beta\left(M_{p}\right)\right)$. Furthermore, the image in qgr- $A$ of any 1 -critical graded $A$-module is a simple object, and so every 1 -critical $A$-module is equal in qgr- $A$ to some $N_{p}$. 
(2) We will reduce to the case of a TCR generated in degree one, where the result is standard. If the result fails, there exists a 1-critical $A$-module $M$ such that (possibly after shifting) $\operatorname{dim} M_{n} \leq 1$ for all $n \geq 0$ but $\operatorname{dim} M_{0}>1$. By replacing $M$ by any submodule generated by a 2-dimensional subspace of $M_{0}$ we may assume that $\operatorname{dim}\left(M_{0}\right)=2$. Write $M=(A \oplus A) / F$.

Now consider $W=\alpha(M) / S(\alpha(M))$. Since $W$ is equal in qgr- $B$ to some $M_{p}$, certainly $\operatorname{dim}\left(W_{n}\right) \leq 1$ for all $n \gg 0$. Moreover, the natural $A$-module map $M \rightarrow W$ must be injective since $M$ is 1 -critical, and so $\operatorname{dim} W_{0} \geq 2$. As $\alpha(M)$ is a factor of $B \oplus B / F B$ it follows that $\operatorname{dim} W_{0}=2$. Unfortunately, $B$ need not be generated in degree 1. However, for $\ell \gg 0$ (indeed $\ell \geq 2$ ) the Veronese ring $C=B^{(\ell)}=$ $B\left(E, \mathscr{H}_{\ell}, \tau^{\ell}\right)$ will be generated in degree one (see [Rogalski 2011, Lemma 3.1(2)]). We claim that $X=W^{(\ell)}$ will still be a critical $C$-module. If not, then, picking an element $0 \neq x \in X_{m}$ in the socle of $X$, we will have $x C_{\geq 1}=0$, and so $x \in W_{m \ell}$ satisfies $x B_{i \ell}=0$ for all $i \geq 1$. Since $B_{i} B_{j}=B_{i+j}$ for all $i, j \gg 0$ [Rogalski 2011, Lemma 3.1(1)], it follows that $x B_{m}=0$ for all $m \gg 0$, contradicting the 1-criticality

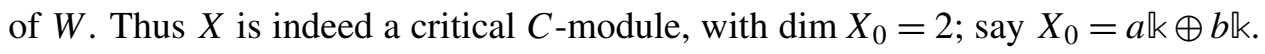

Finally, given $X$, or any 1-critical $C$-module, then [Artin and Van den Bergh 1990] again implies that $\operatorname{dim} X_{n}=1$ for all $n \geq n_{0} \gg 0$. By [Keeler et al. 2005, Proposition 9.2] the map $N \mapsto N_{\geq 1}$ [1] is an automorphism on the set of isomorphism classes of $C$-point modules. Applying the inverse of this map to the shift of $X_{\geq n_{0}}$ shows that the two point modules $a C$ and $b C$ must be equal to this image and hence be isomorphic; say $b C=\phi(a C)$. Set $n=n_{0}+1$. As $\operatorname{dim}_{\mathbb{k}} X_{n}=1$, we can write $C_{n-1}=c \mathbb{k}+\operatorname{ann}_{C}(a)_{n-1}$ for some $c \in C_{n-1}$. Since $\operatorname{ann}_{C}(a)=\operatorname{ann}_{C}(b)$, it follows that $a c=\lambda b c$ for some $\lambda \in \mathbb{k}$. Hence $(a-\lambda b) c=0$, which implies that $(a-\lambda b) C_{n-1}=0$. As $C$ is generated in degree one, this forces $a-\lambda b \in S(X)$. This contradicts the criticality of $X$ and proves the result.

(3) This is immediate from part (2).

(4) If not, pick 1-critical modules $M \nsubseteq M^{\prime}$ such that there is an isomorphism $\gamma: s^{n} M \cong s^{n} M^{\prime} \neq 0$ for some $n>0$. Let $n$ be the smallest integer with this property and then let $W \subset M$ be as large as possible a submodule of $M$ for which $\gamma$ extends to an isomorphism $\gamma: W \rightarrow W^{\prime} \subset M^{\prime}$. Set

$$
N=\frac{M \oplus M^{\prime}}{Z} \quad \text { and } \quad Z=\{(a, \gamma(a)): a \in W\} .
$$

We claim that $N$ is 1-critical. If not, pick a homogeneous element $\left(u, u^{\prime}\right) \in$ $M \oplus M^{\prime}$ such that $\left[\left(u, u^{\prime}\right)+Z\right]$ is a nonzero element of the socle of $N$. If $p \in \mathrm{r}$-ann $(u)$, then $\left(u, u^{\prime}\right) p \in\left(u, u^{\prime}\right) A_{\geq 1} \subseteq Z$. As $u p=0$, this forces $\left(0, u^{\prime}\right) \in Z$, and hence $u^{\prime} p=0$. Similarly, $u^{\prime} p=0$ forces $u p=0$, and hence $\mathrm{r}$-ann $(u)=\mathrm{r}$-ann $\left(u^{\prime}\right)$. Thus, there is an isomorphism $\gamma^{\prime}: u A \cong u^{\prime} A$, which restricts to an isomorphism $\gamma^{\prime \prime}: u A \cap W \rightarrow u^{\prime} A \cap W^{\prime}$. 
We claim that any other isomorphism $\psi: u A \cap W \rightarrow u^{\prime} A \cap W^{\prime}$ must be a scalar multiple of $\gamma^{\prime \prime}$. Put $P=u A \cap W$, which is 1-critical. As we have already proved, $\operatorname{dim}_{\mathbb{k}} P_{n} \leq 1$ for all $n$ with equality for $n \gg 0$. Choose $n$ such that $P_{n} \neq 0$ and fix $0 \neq x \in P_{n}$. Then $x A$ is also 1 -critical and so $x A \doteq P$. Now $\psi(x)=\lambda \gamma^{\prime \prime}(x)$ for some $\lambda \in \mathbb{k}^{\times}$, and this forces $\psi$ to equal $\lambda \gamma^{\prime \prime}$ on all of $x A$. Given homogeneous $y \in P$ with $y \notin x A$, then $0 \neq y z \subseteq x A$ for some $z \in A_{m}, m \gg 0$, and so it is easy to see that this forces $\psi(y)=\lambda \gamma(y)$ also. Thus $\psi=\lambda \gamma^{\prime \prime}$ and the claim follows.

Therefore, possibly after multiplying by a scalar, we can assume that $\gamma^{\prime \prime}=$ $\left.\gamma^{\prime}\right|_{u A \cap W}=\left.\gamma\right|_{u A \cap W}$. Thus, we can extend $\gamma$ to $W+u A$, contradicting the maximality of $W$. Hence $N$ is indeed critical. Finally, as $M \not M^{\prime}$ with $\operatorname{dim} M_{0}=1=\operatorname{dim} M_{0}^{\prime}$, certainly $W \subseteq M_{\geq 1}$, and so $\operatorname{dim} N_{0}=2$, contradicting part (2).

(1) Since the tails $M_{\geq n_{0}}$ of 1-critical $A$-modules are in one-to-one correspondence with the points of $E$, this follows from part (4).

We end the section with a technical consequence of these results for subalgebras of $T$.

Lemma 3.8. Let $U$ be a noetherian cg algebra and $M$ a finitely generated, graded 1-critical right $U$-module. Then $\mathrm{r}-\mathrm{ann}_{U}(M)$ is prime and $\mathrm{r}-\mathrm{ann}_{U}(M)=\mathrm{r}$-ann $U(N)$ for every nonzero submodule $N \subseteq M$.

Proof. This is a standard application of ideal invariance; use, for example, [McConnell and Robson 2001, Corollary 8.3.16 and the proof of (iii) $\Rightarrow$ (iv) of Theorem 6.8.26].

Corollary 3.9. Assume that $T$ satisfies Assumption 2.1 and let $U$ be a $g$-divisible subalgebra of $T_{(g)}$ with $Q_{\mathrm{gr}}(U)=Q_{\mathrm{gr}}(T)$. Suppose that $M$ and $N$ are 1-critical right $U$-modules which are cyclic, generated in degree 0 , with $g \in \mathrm{r}-\operatorname{ann}_{U}(M)$. For some $n \geq 0$ with $M_{n} \neq 0$, suppose that there exists $m \geq 0$ such that $\left(\mathrm{r}-\mathrm{ann}_{U} M_{n}\right)_{\geq m}=$ $\left(\mathrm{r}-\mathrm{ann}_{U} N_{n}\right)_{\geq m}$. Then $M \cong N$.

Proof. By hypothesis, $g^{m} \in\left(\mathrm{r}-\operatorname{ann}_{U} M_{n}\right)_{\geq m}=\left(\mathrm{r}-\operatorname{ann}_{U} N_{n}\right)_{\geq m}$. Then $N_{0} g^{n+m} \subseteq$ $N_{n} g^{m}=0$. As $g$ is central and $N$ is generated by $N_{0}$, it follows that $g^{n+m} \in \mathrm{r}-\mathrm{ann}_{U} N$ and hence $g \in \mathrm{r}-\operatorname{ann}_{U} N$ by Lemma 3.8. Thus, both $M$ and $N$ are modules over $A=\bar{U}$, and to prove the lemma it suffices to consider modules over that ring. By Lemma 2.10, $Q_{\mathrm{gr}}(A)=Q_{\mathrm{gr}}(\bar{T})=k(E)\left[t, t^{-1} ; \tau\right]$, and so $A$ satisfies the hypotheses of Theorem 3.1.

Clearly, $N_{n} \neq 0$. By Corollary 3.7(2), $\operatorname{dim} M_{n}=1=\operatorname{dim} N_{n}$, and so

$$
M_{n} A[n] \cong A / I \quad \text { and } \quad N_{n} A[n] \cong A / J
$$

for some graded right ideals $I, J$. By hypothesis, $I_{\geq m}=J_{\geq m}$. However, as $I / I_{\geq m}$ is finite-dimensional and $A / I$ is 1 -critical, $I / I_{\geq m}$ is the unique largest finite-dimensional submodule of $A / I_{\geq m}=A / J_{\geq m}$. Hence $I=J$ and $M_{n} A \cong N_{n} A$. By Corollary 3.7(4), $M \cong N$. 


\section{Right ideals of $T$ and the rings $T(d)$}

Throughout this section, let $T$ satisfy Assumption 2.1. Our first aim in this section is to describe certain graded right ideals $J$ of $T$ such that $T / J$ is filtered by shifted point modules. In fact, the main method we use in the next section to understand a general subalgebra $U$ of $T$ is to compare its graded pieces with the graded pieces of these right ideals $J$ and their left-sided analogues. The easiest way to construct the required right ideals $J$ is to use some machinery from [Van den Bergh 2001]. The details will appear in a companion paper to this one [RSS 2015].

Definitions 4.1. Given a right ideal $I$ of a cg algebra $R$, the saturation $I^{\text {sat }}$ of $I$ is the sum of the right ideals $L \supseteq I$ with $\operatorname{dim}_{\mathbb{k}} L / I<\infty$. If $I=I^{\text {sat }}$, we say that $I$ is saturated.

Recall that $T / g T \cong B=B(E, M, \tau)$, where $\operatorname{deg} M=\mu$. For divisors $\boldsymbol{b}, \boldsymbol{c}$ on $E$, we write $\boldsymbol{b} \geq \boldsymbol{c}$ if $\boldsymbol{b}-\boldsymbol{c}$ is effective. A list of divisors $\left(\boldsymbol{d}^{0}, \boldsymbol{d}^{1}, \ldots, \boldsymbol{d}^{k-1}\right)$ on $E$ is an allowable divisor layering if $\tau^{-1}\left(\boldsymbol{d}^{i-1}\right) \geq \boldsymbol{d}^{i}$ for all $1 \leq i \leq k-1$. By convention, we define $d^{i}=0$ for all $i \geq k$. Given an allowable divisor layering $\boldsymbol{d}^{\bullet}=\left(\boldsymbol{d}^{0}, \boldsymbol{d}^{1}, \ldots, \boldsymbol{d}^{k-1}\right)$ on $E$, let $J\left(\boldsymbol{d}^{\bullet}\right)$ be the saturated right ideal of $T$ defined in [RSS 2015, Definition 3.4].

We omit the precise definition of $J\left(d^{\bullet}\right)$ because it is technical, and not essential in this paper. Instead, what matters are the following properties of this right ideal, which help explain the name "divisor layering". For any graded right $T$-module $M$, we think of the $B$-module $M g^{j} / M g^{j+1}$ as the $j$-th layer of $M$. Recall that we write $\pi(N)$ for the image of a finitely generated graded $B$-module $N$ in the quotient category qgr- $B$. Recall also from the proof of Corollary 3.7(1) that there is an equivalence of categories coh $E \simeq$ qgr- $B$ given by $\mathscr{F} \mapsto \pi\left(\bigoplus_{n \geq 0} H^{0}\left(E, \mathscr{F} \otimes \mathcal{M}_{n}\right)\right)$.

Lemma 4.2 [RSS 2015, Lemma 3.5]. Let $\boldsymbol{d} \bullet$ be an allowable divisor layering and let $J=J\left(d^{\bullet}\right)$ and $M=T / J$.

(1) If $M^{j}=M g^{j} / M g^{j+1}$, then as objects in qgr- $B$ we have

$$
\pi\left(M^{j}\right) \cong \pi\left(\bigoplus_{n \geq 0} H^{0}\left(E,\left(\mathscr{O}_{E} / \mathscr{O}_{E}\left(-\boldsymbol{d}^{j}\right)\right) \otimes M_{n}\right)\right) .
$$

In particular, the divisor $\boldsymbol{d}^{j}$ determines the point modules that occur in a filtration of $M^{j}$ by (tails of) point modules.

(2) $(\bar{J})^{\text {sat }}=\bigoplus_{n \geq 0} H^{0}\left(E, M_{n}\left(-d^{0}\right)\right)$.

(3) If $\boldsymbol{d}^{\bullet}=(\boldsymbol{d})$ has length 1 , then $J(\boldsymbol{d})=\bigoplus_{n \geq 0}\left\{x \in T_{n}: \bar{x} \in H^{0}\left(E, M_{n}(-\boldsymbol{d})\right)\right\}$.

Note that, as a special case of part (3) of the lemma, if $p \in E$ and $\boldsymbol{d}=p$ then $J(p)$ is simply the right ideal of $T$ such that $P(p)=T / J(p)$ is the point module 
corresponding to the point $p$. (We note that this will coincide with the earlier definition of a point module, should $T$ be generated in degree one.)

We will require primarily the following two special cases of the construction above. Starting now, it will be sometimes convenient to employ the notation

$$
p_{i}=\tau^{-i}(p) \text { for any } p \in E .
$$

Definition 4.4. Given any $p \in E, i \geq 1$, and $0 \leq r \leq d \leq \mu$, we define $Q(i, d, r, p)=$ $J\left(d^{\bullet}\right)$, where

$$
\begin{aligned}
\boldsymbol{d}^{0} & =d p+d p_{1}+\cdots+d p_{i-1}, \\
\boldsymbol{d}^{1} & =d p_{1}+\cdots+d p_{i-1}, \\
& \vdots \\
\boldsymbol{d}^{i-2} & =d p_{i-2}+d p_{i-1}, \\
\boldsymbol{d}^{i-1} & =r p_{i-1} .
\end{aligned}
$$

Intuitively, the divisor layers for $Q$ are in the form of a triangle, but the vanishing in the last layer is allowed to be of lower multiplicity than in the others. The other special case we need is a similar triangle shape which allows for the involvement of points from different orbits.

Definition 4.5. For any divisor $\boldsymbol{d}$ and $k \geq 1$, we define $M(k, \boldsymbol{d})=J\left(\boldsymbol{c}^{\bullet}\right)$, where

$$
\begin{aligned}
\boldsymbol{c}^{0} & =\boldsymbol{d}+\tau^{-1}(\boldsymbol{d})+\cdots+\tau^{-k+1}(\boldsymbol{d}), \\
\boldsymbol{c}^{1} & =\tau^{-1}(\boldsymbol{d})+\cdots+\tau^{-k+1}(\boldsymbol{d}), \\
& \vdots \\
\boldsymbol{c}^{k-1} & =\tau^{-k+1}(\boldsymbol{d}) .
\end{aligned}
$$

It is useful to also define $M(k, \boldsymbol{d})=T$ by convention, for any $k \leq 0$.

Note that $M(k, d p)=Q(k, d, d, p)$ for any $k, d \geq 0$. The right ideals $M(k, \boldsymbol{d})$ are also useful for defining important subalgebras of $T$.

Definition 4.6. For any divisor $\boldsymbol{d}$ with $\operatorname{deg} \boldsymbol{d}<\mu$ we set

$$
T(\boldsymbol{d}):=\bigoplus_{n \geq 0} M(n, \boldsymbol{d})_{n},
$$

which by [RSS 2015, Theorem 5.3(2)] is a $g$-divisible subalgebra of $T$. More generally, for any $\ell \geq 0$ we define

$$
T_{\leq \ell} * T(\boldsymbol{d}):=\bigoplus_{n \geq 0} M\left(n-\ell, \boldsymbol{d}^{\tau^{\ell}}\right)_{n},
$$

which by [RSS 2015, Proposition 5.2(2)] is a right $g$-divisible $T(\boldsymbol{d})$-module. 
When $\operatorname{deg} \boldsymbol{d} \leq \mu-2$, but not in general, the module $T_{\leq \ell} * T(\boldsymbol{d})$ is equal to the right $T(\boldsymbol{d})$-module $T_{\leq \ell} T(\boldsymbol{d}) \subseteq T$ [RSS 2015, Theorem 5.3(6)], so the notation is chosen to suggest multiplication. As is discussed in [Rogalski 2011] and [RSS $2015, \S 5]$, the ring $T(\boldsymbol{d})$ should be thought of as corresponding geometrically to a blowup of $T$ at the divisor $\boldsymbol{d}$.

There are left-sided versions of all of the above definitions and results, because Assumption 2.1 is left-right symmetric. We quickly state these analogues, because there are some nonobvious differences in the statements, which result from the fact that the equivalence of categories $\operatorname{coh} E \simeq B$-qgr has the slightly different form $\mathscr{F} \rightarrow \pi\left(\bigoplus_{n \geq 0} H^{0}\left(E, \mathcal{M}_{n} \otimes \mathscr{F}^{\tau^{n-1}}\right)\right)$. Generally, $\tau^{-1}$ appears in the leftsided results wherever $\tau$ appears in the right-sided version. A list of divisors $\boldsymbol{d}^{\bullet}=\left(\boldsymbol{d}^{0}, \boldsymbol{d}^{1}, \ldots, \boldsymbol{d}^{k-1}\right)$ on $E$ is a left allowable divisor layering if $\tau\left(\boldsymbol{d}^{i-1}\right) \geq \boldsymbol{d}^{i}$ for all $1 \leq i \leq k-1$. We indicate left-sided versions by a prime in the notation. In particular, given a left allowable divisor layering, there is a corresponding saturated left ideal $J^{\prime}\left(\boldsymbol{d}^{\bullet}\right)$ of $T$, defined in [RSS 2015, §6], which satisfies the following analogue of Lemma 4.2.

Lemma 4.7 [RSS 2015, Lemma 6.1]. Let $\boldsymbol{d}^{\bullet}$ be a left allowable divisor layering and let $J^{\prime}=J^{\prime}\left(d^{\bullet}\right)$ and $M=T / J^{\prime}$.

(1) If $M^{j}=M g^{j} / M^{j+1}$ is the $j$-th layer of $M$, then in $B$-qgr we have

$$
\pi\left(M^{j}\right) \cong \pi\left(\bigoplus_{n \geq 0} H^{0}\left(E, M_{n} \otimes\left(\mathscr{O}_{E} / \mathscr{O}_{E}\left(-\tau^{-n+1}\left(\boldsymbol{d}^{j}\right)\right)\right)\right)\right) .
$$

(2) $\left({\overline{J^{\prime}}}^{\mathrm{sat}}=\bigoplus_{n \geq 0} H^{0}\left(E, \mathcal{M}_{n}\left(-\tau^{-n+1}\left(\boldsymbol{d}^{0}\right)\right)\right)\right.$.

(3) If $\boldsymbol{d}^{\bullet}=(\boldsymbol{d})$ has length 1 , then

$$
J^{\prime}(\boldsymbol{d})=\bigoplus_{n \geq 0}\left\{x \in T_{n}: \bar{x} \in H^{0}\left(E, \mu_{n}\left(-\tau^{-n+1}(\boldsymbol{d})\right)\right)\right\} .
$$

Similarly as on the right, as a special case of part (3) we have that $P^{\prime}(p)=$ $T / J^{\prime}(p)$ is the left point module of $T$ corresponding to $p$.

Of course, we also have left-sided analogues of Definitions 4.4 and 4.5, but we only need the former. Namely, given any $p \in E, i \geq 1$, and $0 \leq r \leq d \leq \mu$, we define $Q^{\prime}(i, d, r, p)=J^{\prime}\left(d^{\bullet}\right)$, where

$$
\begin{aligned}
\boldsymbol{d}^{0} & =d p+d p_{-1}+\cdots+d p_{-i+1}, \\
\boldsymbol{d}^{1} & =d p_{-1}+\cdots+d p_{-i+1}, \\
& \vdots \\
\boldsymbol{d}^{i-2} & =d p_{-i+2}+d p_{-i+1}, \\
\boldsymbol{d}^{i-1} & =r p_{-i+1} .
\end{aligned}
$$


The right ideals $Q$ and their left-sided analogues $Q^{\prime}$ will be used below to define filtrations in which every factor is a shifted point module; we will then study how an arbitrary subalgebra $U$ of $T$ intersects such filtrations. The relevant result for this is as follows.

Lemma 4.8 [RSS 2015, Lemma 6.5]. Let $i, r, d, n \in \mathbb{N}$, with $i<n$ and $1 \leq r \leq d \leq \mu$, and $p \in E$. Then:

(1) $Q(i, r, d, p) \subseteq Q(i, r-1, d, p)$, with factor

$$
[Q(i, r-1, d, p) / Q(i, r, d, p)]_{\geq n} \cong P\left(p_{i-n-1}\right)[-n] .
$$

(2) $Q^{\prime}(i, r, d, p) \subseteq Q^{\prime}(i, r-1, d, p)$, with factor

$$
\left[Q^{\prime}(i, r-1, d, p) / Q^{\prime}(i, r, d, p)\right]_{\geq n} \cong P^{\prime}\left(p_{-i+n+1}\right)[-n] .
$$

The left and right ideals defined above are actually closely related. In fact, by [RSS 2015, Proposition 6.8] one always has $Q(i, r, d, p)_{n}=Q^{\prime}\left(i, r, d, p_{i-n}\right)_{n}$, as we will exploit in the next section.

We conclude this section with a review of some important homological concepts.

Definition 4.9. A ring $A$ is called Auslander-Gorenstein if it has finite injective dimension and satisfies the Gorenstein condition: if $p<q$ are nonnegative integers and $M$ is a finitely generated $A$-module, then $\operatorname{Ext}_{A}^{p}(N, A)=0$ for every submodule $N$ of $\operatorname{Ext}_{A}^{q}(M, A)$. Set $j(M)=\min \left\{r: \operatorname{Ext}_{A}^{r}(M, A) \neq 0\right\}$ for the homological grade of $M$. Then an Auslander-Gorenstein ring $A$ of finite Gelfand-Kirillov dimension is called Cohen-Macaulay (or CM) provided that $j(M)+\operatorname{GKdim}(M)=\operatorname{GKdim}(A)$ holds for every finitely generated $A$-module $M$. A cg $\mathbb{k}$-algebra $A$ is called Artin-Schelter (AS) Gorenstein if $A$ has injective dimension $d$ and $\operatorname{dim}_{\mathbb{k}} \operatorname{Ext}_{A}^{j}(\mathbb{k}, A)=\delta_{j, d}$ for all $j \geq 0$. An AS Gorenstein algebra is called AS regular if it is also has finite global dimension $d$.

As the next two results show, many of the algebras appearing in this paper do satisfy these conditions, and this automatically leads to some nice consequences.

Proposition 4.10. Let $R=T(\boldsymbol{d}) \subseteq T$ for some effective divisor $\boldsymbol{d}$ with $\operatorname{deg} \boldsymbol{d} \leq \mu-1$, in the notation of Assumption 2.1. Then the following hold:

(1) $R / g R=B(E, M(-d), \tau)$.

(2) If $\operatorname{deg} \boldsymbol{d}<\mu-1$ then $R$ is generated as an algebra in degree 1, while if $\operatorname{deg} \boldsymbol{d}=\mu-1$ then $R$ is generated as an algebra in degrees 1 and 2.

(3) Both $R$ and $R / g R$ are Auslander-Gorenstein and CM.

(4) $R$ is a maximal order in $Q_{\mathrm{gr}}(R)=Q_{\mathrm{gr}}(T)$.

Proof. Combine [RSS 2015, Theorem 5.3] and [Levasseur 1992, Theorem 6.6]. 
Lemma 4.11. Fix a cg noetherian domain A that is Auslander-Gorenstein and CM. Set $\operatorname{GKdim}(A)=\alpha$.

(1) If $N$ is a finitely generated graded right (or left) A-submodule of $Q=Q_{\mathrm{gr}}(A)$ then $N^{* *}$ is the unique largest submodule $M \subseteq Q$ with $\operatorname{GKdim}(M / N) \leq \alpha-2$.

(2) In particular, there is no graded A-module $A \varsubsetneqq N \subset Q$ with $\operatorname{GKdim}(N / A) \leq$ $\operatorname{GKdim}(A)-2$.

(3) If $J=J^{* *} \neq A$ is a proper reflexive right ideal of $A$ then $A / J$ is $(\alpha-1)$-pure in the sense that $\mathrm{GKdim}(I / J)=\mathrm{GKdim}(A / J)=\alpha-1$ for every nonzero A-module $I / J \subseteq A / J$.

(4) If $N$ is a finitely generated A-module, then $\operatorname{Ext}_{A}^{j(N)}(N, A)$ is a pure module with Gelfand-Kirillov dimension equal to $\operatorname{GKdim}(N)$.

Proof. Part (1) follows from [Björk and Ekström 1990, Theorem 3.6 and Example 3.2]. Parts (2) and (3) are special cases of (1), while part (4) follows by [ibid., Lemma 2.8].

\section{An equivalent $T(d)$}

Throughout this section, $T$ will be an algebra satisfying Assumption 2.1, and we maintain all of the notation introduced in Section 4. In this section we prove that if $U$ is a $g$-divisible graded subalgebra of $T$ with $Q_{\mathrm{gr}}(U)=Q_{\mathrm{gr}}(T)$ then $U$ is an equivalent order to some $T(\boldsymbol{d})$. This should be compared with [Rogalski 2011, Theorem 1.2]: the rings $T(\boldsymbol{d})$ with $\boldsymbol{d}$ effective of degree $<\mu-1$ are precisely the maximal orders $U \subseteq T$ with $Q_{\mathrm{gr}}(U)=Q_{\mathrm{gr}}(T)$ that are generated in degree 1 .

We begin by studying $\bar{U}$ and related subalgebras of $\mathbb{k}(E)\left[t, t^{-1} ; \tau\right]$. We say that two divisors $\boldsymbol{x}$ and $\boldsymbol{y}$ are $\tau$-equivalent if, for every orbit $\mathbb{O}$ of $\tau$ on $E$, one has $\operatorname{deg}\left(\left.\boldsymbol{x}\right|_{\mathbb{O}}\right)=\operatorname{deg}\left(\left.\boldsymbol{y}\right|_{\mathbb{O}}\right)$. Two invertible sheaves $\mathscr{O}_{E}(\boldsymbol{x})$ and $\mathfrak{O}_{E}(\boldsymbol{y})$ are then $\tau$ equivalent if the divisors $\boldsymbol{x}$ and $\boldsymbol{y}$ are $\tau$-equivalent.

Lemma 5.1. Let $\mathcal{N}, \mathcal{N}^{\prime}$ be ample invertible sheaves on $E$ of the same degree. Let $R:=B(E, \mathcal{N}, \tau)$ and $R^{\prime}:=B\left(E, \mathcal{N}^{\prime}, \tau\right)$, and let $F$ be an $\left(R^{\prime}, R\right)$-subbimodule of $\mathbb{k}(E)\left[t, t^{-1} ; \tau\right]$. Then $F_{R}$ is finitely generated if and only if $R^{\prime} F$ is finitely generated. In this case, $\mathcal{N}$ and $\mathcal{N}^{\prime}$ are $\tau$-equivalent.

Proof. Suppose that $F$ is a finitely generated right $R$-module. By [Artin and Van den Bergh 1990, Theorem 1.3] there is an invertible sheaf $\mathscr{F}$ on $E$ so that $F \doteq \bigoplus_{n} H^{0}\left(E, \mathscr{F}_{\mathcal{N}}{ }_{n}\right)$. For $m, n \gg 0$, ampleness ensures that the sheaves $\mathcal{N}_{m}^{\prime}$ and $\mathscr{F}_{\mathcal{N}} \mathcal{N}_{n}$ are generated by their sections and, by construction, those sections are $R_{m}^{\prime}=$ $H^{0}\left(E, \mathcal{N}_{m}^{\prime}\right)$ and $F_{n}=H^{0}\left(E, \mathscr{F}_{\mathcal{N}} \mathcal{N}_{n}\right)$, respectively, again for $m, n \gg 0$. Since $F$ is a left $R^{\prime}$-submodule, $R_{m}^{\prime} F_{n} \subseteq F_{n+m}$ for all $m, n$, and so these observations imply that

$$
\mathcal{N}_{m}^{\prime} \mathscr{F}^{\tau^{m}} \mathcal{N}_{n}^{\tau^{m}} \subseteq \mathscr{F} \mathcal{N}_{n+m}
$$


for all $n, m \gg 0$. By hypothesis, $\mathcal{N}_{m}^{\prime} \mathscr{F}^{\tau^{m}} \mathcal{N}_{n}^{\tau^{m}}$ and $\mathscr{F}_{\mathcal{N}} \mathcal{N}_{n+m}$ have the same degree, and therefore they are equal. In addition, for $n, m \gg 0$ the sheaves $\mathcal{N}_{m}^{\prime}$ and $\mathscr{F}^{\tau}{ }^{m} \mathcal{N}_{n}^{\tau^{m}}$ have degree $\geq 3$. Thus, by [Rogalski 2011, Lemma 3.1], the map

$$
H^{0}\left(E, \mathcal{N}_{m}^{\prime}\right) \otimes H^{0}\left(E, \mathscr{F}^{\tau^{m}} \mathcal{N}_{n}^{\tau^{m}}\right) \rightarrow H^{0}\left(E, \mathcal{N}_{m}^{\prime} \mathscr{F}^{\tau^{m}} \mathcal{N}_{n}^{\tau^{m}}\right)
$$

is surjective. Thus, $R_{m}^{\prime} F_{n}=F_{n+m}$ for all $n, m \gg 0$ and $R_{R^{\prime}} F$ is finitely generated. By symmetry, if $R_{R^{\prime}} F$ is finitely generated then so is $F_{R}$.

In either case, it follows that $\mathcal{N}_{m}^{\prime}=\mathscr{F}_{\mathcal{N}}{ }_{m}\left(\mathscr{F}^{-1}\right)^{\tau^{m}}$ for all $m \gg 0$. The identity $\mathcal{N}^{\prime}\left(\mathcal{N}_{m}^{\prime}\right)^{\tau}=\mathcal{N}_{m+1}^{\prime}$ gives

$\mathcal{N}^{\prime} \mathscr{F}^{\tau} \mathcal{N}_{m}^{\tau}\left(\mathscr{F}^{-1}\right)^{\tau^{m+1}}=\mathcal{N}^{\prime}\left(\mathcal{N}_{m}^{\prime}\right)^{\tau}=\mathcal{N}_{m+1}^{\prime}=\mathscr{F} \mathcal{N}_{m+1}\left(\mathscr{F}^{-1}\right)^{\tau^{m+1}}=\mathscr{F} \mathcal{N} \mathcal{N}_{m}^{\tau}\left(\mathscr{F}^{-1}\right)^{\tau^{m+1}}$.

Rearranging gives $\mathcal{N}^{\prime}=\mathscr{F} \mathcal{N}\left(\mathscr{F}^{-1}\right)^{\tau}$, which is certainly $\tau$-equivalent to $\mathcal{N}$.

We next need two technical results on subalgebras of $B(E, M, \tau)$ that modify the data given by Theorem 3.1.

Notation 5.2. Recall from (4.3) that given a closed point $p \in E$ we write $p_{0}=p$ and $p_{n}=\tau^{-n}(p)$ for all $n \in \mathbb{Z}$. We will also write $\boldsymbol{x}^{\tau}=\tau^{-1}(\boldsymbol{x})$ when $\boldsymbol{x}$ is a divisor (or closed point) on $E$, to distinguish left and right actions, and set $\boldsymbol{x}_{n}=\boldsymbol{x}+\boldsymbol{x}^{\tau}+\cdots+\boldsymbol{x}^{\tau^{n-1}}$.

We start with a routine consequence of Theorem 3.1.

Corollary 5.3. Let $A \subseteq B=B(E, \mathcal{M}, \tau)$ be a cg algebra with $Q_{\mathrm{gr}}(A)=Q_{\mathrm{gr}}(B)$. Then there exist $\boldsymbol{x}, \boldsymbol{y} \in \operatorname{Div}(E)$ and $k \in \mathbb{Z}_{\geq 1}$ so that

$$
A_{n}=H^{0}\left(E, M_{n}\left(-\boldsymbol{y}-\boldsymbol{x}_{n}\right)\right) \quad \text { for all } n \geq k .
$$

Furthermore, $\mu>\operatorname{deg} \boldsymbol{x} \geq 0$, and

for any $n \geq k$ and divisor $\boldsymbol{c}>\boldsymbol{y}+\boldsymbol{x}_{n}$ we have $A_{n} \nsubseteq H^{0}\left(E, M_{n}(-\boldsymbol{c})\right)$.

Proof. By Theorem 3.1, there exist an integer $k \geq 1$, an ideal sheaf $9 y$ and an ample invertible sheaf $\mathcal{N}$ on $E$ so that

$$
A_{n}=H^{0}\left(E, \mathscr{Y}_{\mathcal{N}_{n}}\right) \quad \text { for all } n \geq k .
$$

Let $9=\mathscr{O}_{E}(-\boldsymbol{y})$ for some divisor $\boldsymbol{y}$ and write $\mathcal{N}=M(-\boldsymbol{x})$ for the appropriate divisor $\boldsymbol{x}$ on $E$; thus (5.4) is just a restatement of (5.6). Further, $\operatorname{deg} \boldsymbol{x}=\mu-\operatorname{deg} \mathcal{N}$, which, as $\mathcal{N}$ is ample, implies that $\operatorname{deg} \boldsymbol{x}<\mu$. On the other hand, Riemann-Roch implies that

$$
\mu n=\operatorname{deg} \mathcal{M}_{n}=\operatorname{dim} B_{n} \geq \operatorname{dim} A_{n}=n \operatorname{deg}(\mathcal{N})-\operatorname{deg} \boldsymbol{y}=n(\mu-\operatorname{deg} \boldsymbol{x})-\operatorname{deg} \boldsymbol{y}
$$

for $n \gg 0$. Therefore, $\operatorname{deg} x \geq 0$. 
Finally, since $\mathcal{N}$ is $\tau$-ample, after possibly increasing $k$ we can assume that $M_{n}\left(-\boldsymbol{y}-\boldsymbol{x}_{n}\right)$ is generated by its sections $A_{n}$ for all $n \geq k$ (see for example [Artin and Stafford 1995, Lemma 4.2(1)]). Thus for any larger divisor $\boldsymbol{c}>\boldsymbol{y}+\boldsymbol{x}_{n}$ we will have $H^{0}\left(E, M_{n}(-\boldsymbol{c})\right) \varsubsetneqq H^{0}\left(E, M_{n}\left(-\boldsymbol{y}-\boldsymbol{x}_{n}\right)\right)$. Thus (5.5) also holds.

We next want to modify Corollary 5.3 so that $\boldsymbol{x}$ is replaced by an effective divisor, although this will result in a weaker version of (5.4).

Proposition 5.7. Let $A \subseteq B=B(E, M, \tau)$ be a cg algebra with $Q_{\mathrm{gr}}(A)=Q_{\mathrm{gr}}(B)$. Then there is an effective divisor $\boldsymbol{d}$ on $E$, supported at points with distinct orbits and with $\operatorname{deg} \boldsymbol{d}<\mu$, so that $A$ and $C=B(E, M(-d), \tau)$ are equivalent orders. Moreover, $\boldsymbol{d}$ and $k \in \mathbb{Z}_{\geq 1}$ can be chosen so that

$$
\begin{aligned}
A_{n} \subseteq H^{0}\left(E, M_{n}\left(-\boldsymbol{d}^{\tau^{k}}-\cdots\right.\right. & \left.\left.-\boldsymbol{d}^{\tau^{n-1}}\right)\right) \\
& =H^{0}\left(E, \mathrm{O}_{E}\left(\boldsymbol{d}_{k}\right) \otimes M(-\boldsymbol{d})_{n}\right) \text { for all } n \geq k .
\end{aligned}
$$

Proof. Let $\boldsymbol{x}$ and $\boldsymbol{y}$ be the divisors constructed in the proof of Corollary 5.3, and let $k$ be the integer from that result. Fix an orbit $\mathbb{O}$ of $\tau$ on $E$. By possibly enlarging $k$ we can pick $p \in \mathbb{O}$ so that, using the notation of 5.2,

$$
\begin{aligned}
& \left.\boldsymbol{x}\right|_{\mathbb{O}} \text { is supported on }\left\{p=p_{0}, \ldots, p_{k}\right\}, \quad \text { and } \\
& \left.\boldsymbol{y}\right|_{\mathbb{O}} \text { is supported on }\left\{p_{0}, \ldots, p_{k-1}\right\} .
\end{aligned}
$$

Thus

$$
\left.\boldsymbol{y}\right|_{\mathbb{O}}=\sum_{i=0}^{k-1} y_{i} p_{i} \quad \text { and }\left.\quad \boldsymbol{x}\right|_{\mathbb{O}}=\sum_{i=0}^{k} x_{i} p_{i}
$$

for some integers $y_{i}$ and $x_{j}$. For $n \in \mathbb{N}$ we have

$$
\left.\left(\boldsymbol{x}_{n}\right)\right|_{\mathbb{Q}}=\sum_{i=0}^{k} x_{i}\left(p_{i}+p_{i+1}+\cdots+p_{i+n-1}\right) .
$$

Thus, for $n \geq k$ we calculate that

$$
\begin{array}{r}
\left.\left(\boldsymbol{y}+\boldsymbol{x}_{n}\right)\right|_{\mathbb{O}}=\left(y_{0}+x_{0}\right) p_{0}+\cdots+\left(y_{j}+\sum_{i \leq j} x_{i}\right) p_{j}+\cdots+\left(y_{k-1}+\sum_{i \leq k-1} x_{i}\right) p_{k-1} \\
+\left(\sum_{i \geq 0} x_{i}\right)\left(p_{k}+\cdots+p_{n-1}\right)+\left(\sum_{i \geq 1} x_{i}\right) p_{n}+\cdots+\left(\sum_{i \geq j} x_{i}\right) p_{n+j-1} \\
+\cdots+x_{k} p_{n+k-1} .
\end{array}
$$

Let $e_{p}=\sum x_{i}$. Since $A \subseteq B(E, M, \tau)$, the divisor $\boldsymbol{y}+\boldsymbol{x}_{n}$ is effective for $n \gg 0$, and so

$$
y_{j}+\sum_{i \leq j} x_{i} \geq 0 \quad \text { and } \quad \sum_{i \geq j} x_{i} \geq 0
$$


for all $0 \leq j \leq k$. In particular, $e_{p} \geq 0$. Let $\boldsymbol{d}=\sum_{p} e_{p} p$, where the sum is taken over one closed point $p$ in each orbit $\mathbb{O}$ of $\tau$. Take the maximum of the values of $k$ occurring for the different orbits in the support of $\boldsymbol{x}$ and $\boldsymbol{y}$, and call this also $k$. From (5.10) and (5.11) we see that, on each orbit $\mathbb{O}$ and hence in general,

$$
\boldsymbol{y}+\boldsymbol{x}_{n} \geq \boldsymbol{d}^{\tau^{k}}+\cdots+\boldsymbol{d}^{\tau^{n-1}}
$$

for all $n \geq k$. In other words, (5.8) holds for this $\boldsymbol{d}$ and $k$. By construction, $\operatorname{deg} \boldsymbol{d}=\operatorname{deg} \boldsymbol{x}<\mu$.

Finally, let $\mathcal{N}=\mathcal{M}(-\boldsymbol{x})$ and let $\mathcal{Y}=\mathfrak{O}_{E}(-\boldsymbol{y})$. Let $C=B(E, M(-\boldsymbol{d}), \tau)$ and $C^{\prime}=B(E, \mathcal{N}, \tau)$. Equation (5.8) can be rephrased as saying that

$$
\text { OY } \mathcal{N}_{n} \subseteq \mathcal{M}_{n}\left(-\boldsymbol{d}^{\tau^{k}}-\cdots-\boldsymbol{d}^{\tau^{n-1}}\right)=\mathcal{O}\left(\boldsymbol{d}_{k}\right) \otimes \mathcal{M}(-\boldsymbol{d})_{n} \quad \text { for all } n \geq k .
$$

Thus, for $n_{0} \gg 0$,

$$
C_{\geq n_{0}}^{\prime} \subseteq N=\bigoplus_{n \geq n_{0}} H^{0}\left(E,\left(Y^{-1} \otimes \mathcal{O}\left(\boldsymbol{d}_{k}\right)\right) \otimes M(-\boldsymbol{d})_{n}\right) .
$$

Since $\mathcal{M}(-\boldsymbol{d})$ is $\tau$-ample (because it has positive degree) and $\mathscr{Y}^{-1} \otimes \mathcal{O}\left(\boldsymbol{d}_{k}\right)$ is coherent, [Artin and Stafford 1995, Lemma 4.2(ii)] implies that $N$ is a finitely generated right $C$-module. Hence, so is $C^{\prime} C$. Since $\mathcal{N}=M(-\boldsymbol{x})$ with $\operatorname{deg} \boldsymbol{d}=\operatorname{deg} \boldsymbol{x}$, we can apply Lemma 5.1 to conclude that $C^{\prime} C$ is a finitely generated left $C^{\prime}$-module. Thus $C$ and $C^{\prime}$ are equivalent orders. By the proof of [ibid., Theorem 5.9(2)], $C^{\prime}$ is a finitely generated right $A$-module. Thus $C^{\prime}$ and $A$ are equivalent orders, and so $C$ and $A$ are also equivalent.

Definition 5.12. We say that $(\boldsymbol{y}, \boldsymbol{x}, k)$ as given by Corollary 5.3 is geometric data for $A$. If (5.9) holds for $p \in \mathbb{O}$, we say $p$ is a normalised orbit representative for this data, and we say that $\boldsymbol{d}=\sum e_{p} p$ is a normalised divisor for $(\boldsymbol{y}, \boldsymbol{x}, k)$. To avoid trivialities, the only orbits considered here are the (finite number of) orbits containing the support of $\boldsymbol{x}$ and $\boldsymbol{y}$. By construction, $\operatorname{deg} \boldsymbol{d}=\operatorname{deg} \boldsymbol{x}<\mu$.

We now use these results to study subalgebras of $T$, and begin with a general idea of the strategy. Let $U$ be a $g$-divisible graded subalgebra of $T$ so that $Q_{\mathrm{gr}}(U)=$ $Q_{\mathrm{gr}}(T)$. By Proposition 2.9, $U$ is automatically a finitely generated, noetherian $\mathbb{k}$-algebra, so the earlier results of the paper are available to us. Let $(\boldsymbol{y}, \boldsymbol{x}, k)$ be geometric data for $\bar{U}$ and let $\boldsymbol{d}$ be a normalised divisor for $(\boldsymbol{y}, \boldsymbol{x}, k)$. We will show that $U$ and $T(\boldsymbol{d})$ are equivalent orders.

Recall that the right $T(\boldsymbol{d})$-module $T_{\leq k} * T(\boldsymbol{d})=\bigoplus_{n \geq 0} M\left(n-k, \boldsymbol{d}^{\tau^{k}}\right)_{n}$ from Definition 4.6 is $g$-divisible, with

$$
\overline{T_{\leq k} * T(\boldsymbol{d})}=\bigoplus_{n \geq 0} H^{0}\left(E, \mu_{n}\left(-\boldsymbol{d}^{\tau^{k}}-\cdots-\boldsymbol{d}^{\tau^{n-1}}\right)\right),
$$


by Lemma 4.2. In other words, by (5.8),

$$
\bar{U} \subseteq \overline{T_{\leq k} * T(\boldsymbol{d})}
$$

Our next goal is to show that this holds without working modulo $g$ : that is, that

$$
U \subseteq T_{\leq k} * T(\boldsymbol{d})
$$

This will force $\widehat{U T(\boldsymbol{d})}$ to be finitely generated as a right $T(\boldsymbol{d})$-module, which is a key step towards proving that $U$ and $T(\boldsymbol{d})$ are equivalent orders.

Suppose therefore that (5.13) fails, and so $U_{n_{0}} \nsubseteq\left(T_{\leq k} * T(\boldsymbol{d})\right)_{n_{0}}$ for some $n_{0}$. Necessarily, $n_{0}>k$. We will find a right $T$-ideal $Q(i, r, e, p)$ and a left $T$-ideal $Q^{\prime}(i, r, e, q)$ such that if we set $I=U \cap Q(i, r, e, p)$ and $J=U \cap Q^{\prime}(i, r, e, q)$ then $U / I$ and $U / J$ are isomorphic to point modules in large degree. Further, we can choose $p$ and $q$ so that $I_{n_{0}}=J_{n_{0}}$. However, Corollary 3.9 can be used to derive precise formulæ for $I_{n_{0}}$ and $J_{n_{0}}$, and we will see that these formulæ are inconsistent, leading to a final contradiction.

In the next few results, we carry out this argument, using induction and a filtration of $T$ by the right ideals $Q$ defined in Section 4. Recall the definition of $I^{\text {sat }}$ from Definitions 4.1, and the definitions of $J\left(\boldsymbol{d}^{\bullet}\right), Q(i, r, d, p)$, and their left-sided analogues from Section 4.

Lemma 5.14. Let $U$ be a g-divisible graded subalgebra of $T$ with $Q_{\mathrm{gr}}(U)=$ $Q_{\mathrm{gr}}(T)$. Suppose that $n>i \geq 1,1 \leq r \leq e \leq \mu$, and $j \in \mathbb{Z}$. Suppose further that

(A) $U_{\geq n} \subseteq Q\left(i, r-1, e, p_{j}\right)$, but $U_{n} \nsubseteq \subseteq\left(i, r, e, p_{j}\right)$; and

(B) $\bar{U}_{m} \not \Phi \overline{J\left(p_{i+j-n-1}\right)_{m}}=H^{0}\left(E, M_{m}\left(-p_{i+j-n-1}\right)\right)$ for all $m \geq n$.

Let $I=U \cap Q\left(i, r, e, p_{j}\right)$, and let $M=U / I^{\mathrm{sat}}$. Then:

(1) $M_{n} \neq 0$.

(2) $M$ is 1-critical and $M g=0$.

(3) $\left(\mathrm{r}-\mathrm{ann}_{U}\left(M_{n}\right)\right)_{m}=\left(U \cap J\left(p_{i+j-n-1}\right)\right)_{m}$ for all $m \gg 0$.

Proof. (1) Let $L=U / I$, so that $M=L / L^{\prime}$, where $L^{\prime}$ is the largest finite-dimensional submodule of $L$. Since $n>i$, it follows from hypothesis (A) and Lemma 4.8(1) that $\operatorname{dim} L_{m} \leq 1$ for all $m \geq n$ and that

$$
U_{n} J\left(p_{i+j-n-1}\right) \subseteq Q\left(i, r, e, p_{j}\right) .
$$

If $M_{n}=0$, then $L_{n} U_{m}=0$ for all $m \gg 0$. Then $U_{n} U_{m} \subseteq Q\left(i, r, e, p_{j}\right)$ for all $m \gg 0$. By hypothesis (B), $U_{m}+J\left(p_{i+j-n-1}\right)_{m}=T_{m}$ for $m \geq n$, so $U_{n} T_{m} \subseteq Q\left(i, r, e, p_{j}\right)$ for $m \gg 0$ also. Since $Q\left(i, r, e, p_{j}\right)$ is a saturated right $T$-ideal, $U_{n} \subseteq Q\left(i, r, e, p_{j}\right)$, contradicting the hypotheses. 
(2) By Lemma 4.2(3), $g \in J\left(p_{i+j-n-1}\right)$, whence $M_{\geq n} \cdot g=0$ and $\mathrm{r}-\operatorname{ann}_{U}(M) \supseteq$ $U_{\geq n} g=g U_{\geq n}$. By construction, $M$ has no finite-dimensional submodules, and so $M g=0$. Thus $M$ is a $\bar{U}$-module. Also, $\operatorname{dim}_{\mathbb{k}} M_{m} \leq \operatorname{dim}_{\mathbb{k}} L_{m} \leq 1$ for all $m \geq n$ and $M \neq 0$ by part (1), so $\operatorname{GKdim}(M)=1$. Since $M$ is noetherian, it has a $\bar{U}$ submodule $M^{\prime}$ maximal with respect to the property $\operatorname{GKdim}\left(M / M^{\prime}\right)=1$. Then $M / M^{\prime}$ is 1-critical. However, by Corollary 3.7(2) any 1-critical $\bar{U}$-module $N$ has $\operatorname{dim} N_{m}=1$ for all $m \gg 0$. Thus $M^{\prime}$ is finite-dimensional; hence $M^{\prime}=0$ and $M$ is 1-critical.

(3) Since $M$ is 1-critical, its cyclic submodule $N=M_{n} U$ must also be 1-critical. Thus $\operatorname{dim}_{\mathbb{k}} M_{n}=1=\operatorname{dim}_{\mathbb{k}} N_{n}$ for $n \gg 0$, forcing $M \doteq N$. In particular, we must have r-ann ${ }_{U}\left(M_{n}\right)_{m} \varsubsetneqq U_{m}$ for all $m \gg 0$. By (5.15), r-ann $U\left(M_{n}\right) \supseteq U \cap J\left(p_{i+j-n-1}\right)$. Now Lemma 4.2(3) implies that $J\left(p_{i+j-n-1}\right)_{m}$ has codimension 1 in $T_{m}$ for all

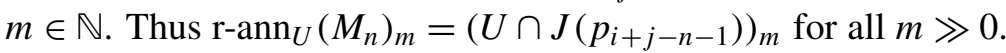

Corollary 5.16. Assume that we have the hypotheses of Lemma 5.14. Assume in addition to $(A),(B)$ that we have $e<\mu$ and

(C) $\bar{U}_{\geq n} \subseteq \overline{J\left(e p_{j+i-1}\right)}=H^{0}\left(E, M_{n}\left(-e p_{j+i-1}\right)\right)$, but

$$
\bar{U}_{n} \nsubseteq \overline{J\left((e+1) p_{j+i-1}\right)}=H^{0}\left(E, M_{n}\left(-(e+1) p_{j+i-1}\right)\right) .
$$

Then $U_{n} \cap Q\left(i, r, e, p_{j}\right)=U_{n} \cap J\left((e+1) p_{i+j-1}\right)$.

Proof. Let

$$
I=\left(U \cap Q\left(i, r, e, p_{j}\right)\right)^{\mathrm{sat}} \quad \text { and } \quad M=U / I .
$$

Similarly, let

$$
H=\left(U \cap J\left((e+1) p_{i+j-1}\right)\right)^{\text {sat }} \text { with } \quad N=U / H .
$$

Note that $Q\left(1, d, d, p_{j+i-1}\right)=J\left(d p_{j+i-1}\right)$ for any $d$. Also, since $g \in J\left(d p_{j+i-1}\right)$, hypothesis (C) is equivalent to $U_{\geq n} \subseteq J\left(e p_{j+i-1}\right)$ but $U_{n} \nsubseteq J\left((e+1) p_{j+i-1}\right)$. Thus, hypothesis (C) implies that the hypothesis (A) of Lemma 5.14 also holds for $\left(i^{\prime}, r^{\prime}, e^{\prime}\right)=(1, e+1, e+1)$ and $j^{\prime}=i+j-1$. Also, hypothesis (B) for these values is the same as hypothesis (B) for the old values. Since $e<\mu$, the hypotheses of Lemma 5.14 hold for $\left(i^{\prime}, r^{\prime}, e^{\prime}\right)$.

We may now apply Lemma 5.14 to $M$ and $N$. Thus, $M_{n}, N_{n} \neq 0$, both $M, N$ are 1-critical and killed by $g$, and $\mathrm{r}-\operatorname{ann}_{U}\left(M_{n}\right)$ and $\mathrm{r}-\operatorname{ann}_{U}\left(N_{n}\right)$ are both equal to $U \cap J\left(p_{i+j-n-1}\right)$ in large degree. By Corollary 3.9, we have $M \cong N$ and so $I=H$. Thus, since $U_{n} \cap Q\left(i, r, e, p_{j}\right)$ and $U_{n} \cap J\left((e+1) p_{i+j-1}\right)$ are already saturated in degree $n$ by Lemma 5.14(1), we have

$$
U_{n} \cap Q\left(i, r, e, p_{j}\right)=I_{n}=H_{n}=U_{n} \cap J\left((e+1) p_{i+j-1}\right) .
$$

We also need the left-sided versions of the two preceding results. Since the statements and proofs of these are largely symmetric, we give a combined statement 
of the left-sided versions, with an abbreviated proof. We note that a consequence of [RSS 2015, Lemmas 3.5 and 6.1] is that

$$
\begin{aligned}
& J^{\prime}\left(d p_{j}\right)_{n}=J\left(d p_{j+n-1}\right)_{n} \quad \text { and } \\
& \overline{J^{\prime}\left(d p_{j}\right)_{n}}=H^{0}\left(E, M_{n}\left(-d p_{j+n-1}\right)\right)=\overline{J\left(d p_{j+n-1}\right)_{n}} .
\end{aligned}
$$

Lemma 5.18. Let $U$ be a g-divisible graded subalgebra of $T$ with $Q_{\mathrm{gr}}(U)=$ $Q_{\mathrm{gr}}(T)$. Suppose that $n>i \geq 1,1 \leq r \leq e<\mu$, and $h \in \mathbb{Z}$. Suppose further that

( $\left.\mathrm{A}^{\prime}\right) U_{\geq n} \subseteq Q^{\prime}\left(i, r-1, e, p_{h}\right)$, but $U_{n} \nsubseteq Q^{\prime}\left(i, r, e, p_{h}\right)$;

(B') $\bar{U}_{m} \nsubseteq \overline{J^{\prime}\left(p_{h-i+n+1}\right)_{m}}=H^{0}\left(E, M_{n}\left(-p_{h-i+n+m}\right)\right)$ for $m \geq n$; and

(C') $\bar{U}_{\geq n} \subseteq \overline{J^{\prime}\left(e p_{h-i+1}\right)}, \quad$ but

$$
\bar{U}_{n} \not \overline{J^{\prime}\left((e+1) p_{h-i+1}\right)_{n}}=H^{0}\left(E, M_{n}\left(-(e+1) p_{h-i+n}\right)\right) .
$$

Then $U_{n} \cap Q^{\prime}\left(i, r, e, p_{h}\right)=U_{n} \cap J^{\prime}\left((e+1) p_{h-i+1}\right)$.

Proof. The equalities in $\left(\mathrm{B}^{\prime}\right),\left(\mathrm{C}^{\prime}\right)$ follow from (5.17). The rest of the proof is symmetric to the proofs of Lemma 5.14 and Corollary 5.16. In particular, one uses part (2) of Lemma 4.8 in place of part (1).

The next result is the heart of the proof that $U$ and $T(\boldsymbol{d})$ are equivalent orders.

Proposition 5.19. Let $U$ be a g-divisible cg subalgebra of $T$ with $Q_{\mathrm{gr}}(U)=Q_{\mathrm{gr}}(T)$. Let $(\boldsymbol{y}, \boldsymbol{x}, k)$ be geometric data for $\bar{U}$ and let $\boldsymbol{d}=\sum e_{p} p$ be a normalised divisor for this data. Then

$$
U \subseteq T_{\leq k} * T(\boldsymbol{d})
$$

Proof. If $\boldsymbol{d}=0$ the result is trivial, so we may assume that $\boldsymbol{d}>0$. Suppose that $U \nsubseteq T_{\leq k} * T(\boldsymbol{d})$.

By [RSS 2015, Lemma 6.6], $T_{\leq k} * T(\boldsymbol{d})=\bigcap_{p} T_{\leq k} * T\left(e_{p} p\right)$, where the intersection is over the normalised orbit representatives $p$. Thus there is some such $p$ so that $U \nsubseteq T_{\leq k} * T\left(e_{p} p\right)$. Let $e=e_{p}<\mu$. By [RSS 2015, Lemma 6.6], again, for $n \in \mathbb{N}$ we have

$$
\left(T_{\leq k} * T(e p)\right)_{n}=\bigcap\left\{Q\left(i, r, e, p_{j}\right)_{n}: i \geq 1, k \leq j \leq n-i, 1 \leq r \leq e\right\} .
$$

Thus, there are $i \geq 1,1 \leq r \leq e$, and $n, j \in \mathbb{N}$ with $1 \leq k \leq j \leq n-i$ such that

$$
U_{n} \nsubseteq Q\left(i, r, e, p_{j}\right)_{n} .
$$

Without loss of generality we can assume that $i$ is minimal such that we can achieve this for some such $n, j, r$. Note that $i \geq 2$, since $\overline{Q\left(1, r, e, p_{j}\right)}=H^{0}\left(E, M\left(-r p_{j}\right)\right)$ by Lemma 4.2(2), and the sections in $\overline{U_{n}}$ vanish to multiplicity $e$ at $p_{j}$ by (5.10). Then choose $r$ minimal (for this $i$ ) so that (5.20) holds for some such $n, j$. Intuitively, we are finding a "divisor triangle" of minimal size $i$ such that the corresponding 
right ideal does not contain $U_{n}$, with deepest layer vanishing condition in this triangle to be of multiplicity $r$ as small as possible.

Claim 1. $U_{n} \cap Q\left(i, r, e, p_{j}\right)=U_{n} \cap J\left((e+1) p_{j+i-1}\right)$.

Proof. We check the hypotheses of Corollary 5.16. Hypothesis (A) follows by minimality of $r$ when $r>1$. When $r=1$, then we need $U_{\geq n} \subseteq Q\left(i, 0, e, p_{j}\right)$. Now, by [RSS 2015, (6.7)],

$$
Q\left(i, 0, e, p_{j}\right)=Q\left(i-1, e, e, p_{j}\right) \cap Q\left(i-1, e, e, p_{j+1}\right) .
$$

Since $U_{\geq n}$ is contained in both $Q\left(i-1, e, e, p_{j}\right)$ and $Q\left(i-1, e, e, p_{j+1}\right)$ by the minimality of $i$, hypothesis (A) holds in this case as well.

Note that, by (5.5), the equation (5.10) gives exactly the vanishing (with multiplicities) at points on the $\tau$-orbit of $p$ for the sections in $\bar{U}_{n} \subseteq H^{0}\left(E, \mu_{n}\right)$. In particular, (B) holds because $i+j-n-1<0$. Similarly, (C) holds by (5.10) since $k \leq j+i-1 \leq n-1$. Thus Corollary 5.16 gives the result.

Claim 2. $U_{n} \cap Q^{\prime}\left(i, r, e, p_{h}\right)=U_{n} \cap J^{\prime}\left((e+1) p_{h-i+1}\right)$ for $h=j+i-n$.

Proof. This similarly follows from Lemma 5.18 once we verify the hypotheses of that result. For $\left(\mathrm{B}^{\prime}\right)$, note that $h-i+n+m=j+m \geq k+m$ and use (5.10). Hypothesis $\left(\mathrm{C}^{\prime}\right)$ follows again from (5.10) since $h-i+n=j$ satisfies $k \leq j \leq n-1$.

It remains to verify $\left(\mathrm{A}^{\prime}\right)$. We will use the equality

$$
Q(k, r, m, p)_{n}=Q^{\prime}\left(k, r, m, p_{k-n}\right)_{n},
$$

proven in [RSS 2015, Proposition 6.8(3)]. Thus, $U_{n} \nsubseteq \subseteq Q^{\prime}\left(i, r, e, p_{h}\right)_{n}$. Now let $n^{\prime} \geq n$. Suppose that $r>1$. The minimality hypothesis on $r$ means that, for any $j^{\prime}$ with $k \leq j^{\prime} \leq n^{\prime}-i$, we have $U_{n^{\prime}} \subseteq Q\left(i, r-1, e, p_{j^{\prime}}\right)_{n^{\prime}}$. In particular, since $k \leq j+n^{\prime}-n \leq n^{\prime}-i$, we have

$$
U_{n^{\prime}} \subseteq Q\left(i, r-1, e, p_{j+n^{\prime}-n}\right)_{n^{\prime}}=Q^{\prime}\left(i, r-1, e, p_{h}\right)_{n^{\prime}}
$$

by (5.22). Thus $U_{\geq n} \subseteq Q^{\prime}\left(i, r-1, e, p_{h}\right)$. If instead $r=1$, then

$$
\begin{aligned}
Q^{\prime}\left(i, 0, e, p_{h}\right)_{n^{\prime}} & =Q\left(i, 0, e, p_{j-n+n^{\prime}}\right) \\
& =Q\left(i-1, e, e, p_{j-n+n^{\prime}}\right) \cap Q\left(i-1, e, e, p_{j-n+n^{\prime}+1}\right)
\end{aligned}
$$

by (5.21) and (5.22). But $U_{n^{\prime}}$ is contained in both $Q\left(i-1, e, e, p_{j-n+n^{\prime}+1}\right)$ and $Q\left(i-1, e, e, p_{j-n+n^{\prime}}\right)$ by minimality of $i$. Thus $U_{\geq n} \subseteq Q^{\prime}\left(i, r-1, e, p_{h}\right)$ in this case as well, and $\left(\mathrm{A}^{\prime}\right)$ holds as needed.

Claim 3. $U_{n} \cap Q\left(i, r, e, p_{j}\right)=U_{n} \cap J\left((e+1) p_{j}\right)$. 
Proof. As in the proof of Claim 2, we have $Q\left(i, r, e, p_{j}\right)_{n}=Q^{\prime}\left(i, r, e, p_{j+i-n}\right)_{n}$, and so that claim gives

$$
\begin{aligned}
U_{n} \cap Q\left(i, r, e, p_{j}\right) & =U_{n} \cap Q^{\prime}\left(i, r, e, p_{j+i-n}\right) \\
& =U_{n} \cap J^{\prime}\left((e+1) p_{j-n+1}\right) \\
& =U_{n} \cap J\left((e+1) p_{j}\right),
\end{aligned}
$$

where we use (5.17) in the last step.

We can now complete the proof of Proposition 5.19. Combining Claims 1 and 3, we have

$$
U_{n} \cap J\left((e+1) p_{j}\right)=U_{n} \cap Q\left(i, r, e, p_{j}\right)=U_{n} \cap J\left((e+1) p_{i+j-1}\right) .
$$

Recall that $\bar{U}_{n}=H^{0}\left(E, \mathcal{M}_{n}\left(-\boldsymbol{y}-\boldsymbol{x}_{n}\right)\right)$ and $i \geq 2$. Thus, by (5.10) and (5.5) we see that, after taking the image of (5.23) in $B$, the right-hand side vanishes to order $e$ at $p_{j}$, while the left-hand side vanishes to order $e+1$ at $p_{j}$. This contradiction completes the proof of the proposition.

We can now quickly prove our first main theorem.

Theorem 5.24. Let $U$ be a g-divisible graded subalgebra of $T$ with $Q_{\mathrm{gr}}(U)=$ $Q_{\mathrm{gr}}(T)$. Then there is an effective divisor $\boldsymbol{d}$ on $E$, supported on points with distinct orbits and with $\operatorname{deg} \boldsymbol{d}<\mu$, so that $U$ is an equivalent order to $T(\boldsymbol{d})$.

In more detail, for some $d$ the $(U, T(d))$-bimodule $M=\widehat{U T(\boldsymbol{d})}$ is a finitely generated g-divisible right $T(\boldsymbol{d})$-module with $M T=T$. Set $W=\operatorname{End}_{T(\boldsymbol{d})}(M)$. Then $U \subseteq W \subseteq T$, the bimodule $M$ is finitely generated as a left $W$-module, while $W, U$, and $T(\boldsymbol{d})$ are equivalent orders.

Remark 5.25. Recall from Lemma 2.10 that, if $U$ be a $g$-divisible graded subalgebra of $T$ with $D_{\mathrm{gr}}(U)=D_{\mathrm{gr}}(T)$, then $Q_{\mathrm{gr}}(U)=Q_{\mathrm{gr}}(T)$ also holds. However, some condition on quotient rings is required for the theorem, since clearly $U=\mathbb{k}[g]$ is not equivalent to any $T(\boldsymbol{d})$.

Proof. By Lemma 2.10, $Q_{\mathrm{gr}}(\bar{U})=Q_{\mathrm{gr}}(\bar{T})$ and so we can apply Proposition 5.7 to $A=\bar{U}$. Let $\boldsymbol{d}, k$ be as defined there; thus if $R=T(\boldsymbol{d})$ then $\bar{U}$ and $\bar{R}$ are equivalent orders. By Proposition 5.19, $U \subseteq T_{\leq k} * R$.

Let $M=\widehat{U R}$ and $W=\operatorname{End}_{R}(M)$. By [RSS 2015, Theorem 5.3(5)], $T_{\leq k} * R$ is a noetherian right $R$-module and so $M \subseteq T_{\leq k} * R$ is a finitely generated right $R$-module. Clearly $M T=T$ since $1 \in M \subseteq T$ and so $W \subseteq T$. Thus, by Lemma 2.12(3), ${ }_{W} M$ is finitely generated, and so $W$ and $R$ are equivalent orders. A routine calculation shows that $M$ is a left $U$-module and so $U \subseteq W$.

Consider the $(\bar{W}, \bar{R})$-bimodule $\bar{M}$. This is finitely generated on both sides, since the same is true of ${ }_{W} M_{R}$. Thus $\bar{W}$ and $\bar{R}$ are equivalent orders, which, as $\bar{R}$ and $\bar{U}$ are equivalent orders, implies that $\bar{W}$ and $\bar{U}$ are likewise. Finally, as $U \subseteq W \subseteq T$, 
the hypotheses of the theorem ensure that $Q_{\mathrm{gr}}(U)=Q_{\mathrm{gr}}(W)=Q_{\mathrm{gr}}(T)$. Thus, by Proposition 2.16, $U$ and $W$ are equivalent orders, and hence so are $U$ and $R$.

Corollary 5.26. Suppose that $\boldsymbol{u}$ and $\boldsymbol{v}$ are two effective, $\tau$-equivalent divisors with degree $\operatorname{deg} \boldsymbol{u} \leq \mu-1$. Then $T(\boldsymbol{u})$ and $T(\boldsymbol{v})$ are equivalent orders.

Proof. Consider the construction of the divisor $\boldsymbol{d}$ in Theorem 5.24 starting from the algebra $U=T(\boldsymbol{u})$. Thus $\boldsymbol{d}=\sum e_{p} p$ is the divisor constructed in Proposition 5.7 and there is considerable flexibility in its choice. To begin, in the proof of Equation (5.4), one sees that $\boldsymbol{y}=0$ and $\boldsymbol{x}=\boldsymbol{u}$. For each orbit $\mathbb{O}$ of $\tau$, a point $p$ is then chosen such that $\left.\boldsymbol{u}\right|_{\mathbb{O}}$ is supported on $X_{\mathbb{O}}=\left\{p_{0}=p(\mathbb{O}), p_{1}, \ldots, p_{k}\right\}$. For each such orbit, we can replace $p_{0}$ by some $p_{-r}$ and increase $k$ so that both $\left.\boldsymbol{u}\right|_{\mathbb{O}}$ and $\left.\boldsymbol{v}\right|_{\mathbb{O}}$ are supported on $X_{\mathbb{O}}$. Then $\boldsymbol{d}=\sum_{\mathbb{O}} e_{p} p(\mathbb{O})$, for these choices of points $p(\mathbb{O})$, and $e_{p}=\operatorname{deg}\left(\left.\boldsymbol{u}\right|_{\mathbb{O}}\right)$. As $\boldsymbol{u}$ and $\boldsymbol{v}$ are $\tau$-equivalent, $\operatorname{deg}\left(\left.\boldsymbol{u}\right|_{\mathbb{O}}\right)=\operatorname{deg}\left(\left.\boldsymbol{v}\right|_{\mathbb{O}}\right)$ for each orbit $\mathbb{O}$, and hence the divisor $\boldsymbol{d}$ is the same whether we started with $T(\boldsymbol{u})$ or $T(\boldsymbol{v})$. Hence, by Theorem 5.24, $T(\boldsymbol{u})$ and $T(\boldsymbol{v})$ are both equivalent to $T(\boldsymbol{d})$ and hence to each other.

Remark 5.27. One disadvantage of Theorem 5.24 is that the $(U, T(\boldsymbol{d}))$-bimodule $M$ constructed there need not be finitely generated as a left $U$-module. Using [McConnell and Robson 2001, Proposition 3.1.14] and the fact that our rings are noetherian, one can easily produce such a bimodule. However, this typically lacks the extra structure inherent in $M$ (notably that $M T=T$ ) and so is less useful for our purposes. As will be seen in the next section, this problem disappears when one works with maximal orders (see Corollary 6.6, for example) and this will in turn give extra information about the structure of such an algebra.

\section{On endomorphism rings of $T(d)$-modules}

Given a $g$-divisible algebra $U \subseteq T$, Theorem 5.24 provides a module $M$ over some blowup $T(\boldsymbol{d})$ with $U \subseteq \operatorname{End}_{T(\boldsymbol{d})}(M)$. In this section, we reverse this procedure by obtaining detailed properties of such endomorphism rings (see Proposition 6.4 and Theorem 6.7). These results provide important information about the structure of maximal $T$-orders that will in turn be refined over the next two sections to prove the main result Theorem 1.2 from the introduction.

We begin with an expanded version of a definition from the introduction.

Definition 6.1. Let $U \subseteq V$ be Ore domains with the same quotient ring $Q(U)$. We say that $U$ is a maximal $V$-order if there exists no order $U \varsubsetneqq U^{\prime} \subseteq V$ that is equivalent to $U$. We note that if $U$ and $V$ are graded (in which case requiring that $Q_{\mathrm{gr}}(U)=Q_{\mathrm{gr}}(V)$ is sufficient) then this is the same as being maximal among graded orders equivalent to $U$ and contained in $V$. Indeed, suppose that $U$ has the latter property, but that $U \subsetneq A \subseteq V$ for some equivalent order $A$. If $A$ is given the filtration induced from the graded structure of $V$, then the associated graded ring 
gr $A$ will still satisfy $U \subsetneq$ gr $A \subseteq V$ and be equivalent to $U$, giving the required contradiction.

When $V=Q(U)$, or $V=Q_{\mathrm{gr}}(U)$ if $U$ is graded, a maximal $V$-order is simply called a maximal order.

We are mostly interested in maximal $T$-orders. We introduce this concept because maximal $T$-orders need not be maximal orders (see Proposition 10.3), although the difference is not large (see Corollary 6.6). We first want to study the endomorphism ring $\operatorname{End}_{T(\boldsymbol{d})}(M)$ arising from Theorem 5.24, and we begin with two useful lemmas.

Lemma 6.2. Let $A$ be a noetherian domain with quotient division ring $D$. If $N$ is a finitely generated right A-submodule of $D$ then $\operatorname{End}_{A}\left(N^{* *}\right)$ is the unique maximal order among orders containing and equivalent to $\operatorname{End}_{A}(N)$.

Proof. This is what is proved in [Cozzens 1976, Theorem 2.7], since $\operatorname{End}_{A}\left(N^{*}\right)=$ $\operatorname{End}_{A}\left(N^{* *}\right)$.

Lemma 6.3. Let $A$ and $B$ be rings such that $A$ is left noetherian and suppose that $M$ is an (A, B)-bimodule that is finitely generated on both sides, and that $N$ is a finitely generated right $B$-module. Then $\operatorname{Hom}_{B}(N, M)$ is a finitely generated left $A$-module. In particular, $\operatorname{End}_{B}(M)$ is a finitely generated left $A$-module, and if $B$ is left noetherian then $N^{*}=\operatorname{Hom}_{B}(N, B)$ is a finitely generated left $B$-module.

Proof. A surjective $B$-module homomorphism $B^{\oplus n} \rightarrow N$ induces an injective left $A$-module homomorphism $\operatorname{Hom}_{B}(N, M) \hookrightarrow \operatorname{Hom}_{B}\left(B^{\oplus n}, M\right) \cong M^{\oplus n}$. Since $M$ is a noetherian left $A$-module, $\operatorname{Hom}_{B}(N, M)$ is a finitely generated left $A$-module.

We are now ready to prove the first significant result of the section. Until further notice, all duals $N^{*}$ will be taken as $R$-modules, for $R=T(\boldsymbol{d})$.

Proposition 6.4. Let $\boldsymbol{d}$ be an effective divisor on $E$ with $\operatorname{deg} \boldsymbol{d}<\mu$ and let $R=T(\boldsymbol{d})$. Let $M \subseteq T_{(g)}$ be a g-divisible finitely generated graded right $R$-module with $M T=T$ and set $W=\operatorname{End}_{R}(M)$ and $F=\operatorname{End}_{R}\left(M^{* *}\right)$. Then:

(1) $F, V=F \cap T$ and $W$ are g-divisible algebras with $Q_{\mathrm{gr}}(W)=Q_{\mathrm{gr}}(V)=$ $Q_{\mathrm{gr}}(F)=Q_{\mathrm{gr}}(T)$.

(2) $F$ is the unique maximal order containing and equivalent to $W$, while $V$ is the unique maximal $T$-order containing and equivalent to $W$.

(3) There is an ideal $K$ of $F$ with $K \subseteq W$ and GKdim $F / K \leq 1$.

(4) $R=\operatorname{End}_{W}(M)=\operatorname{End}_{F}\left(M^{* *}\right)$.

Proof. Since $Q_{\mathrm{gr}}(R)=Q_{\mathrm{gr}}(T)$ by Proposition 4.10, clearly the same is true for $W, V$ and $F$. As in (2.11), given a right $R$-module $N \subset Q_{\mathrm{gr}}(R)$ we identify

$$
N^{*}=\operatorname{Hom}_{R}(N, R)=\left\{\theta \in Q_{\mathrm{gr}}(R): \theta N \subseteq R\right\},
$$


and similarly for left modules. By Lemma $2.12(3), W$ is $g$-divisible and ${ }_{W} M$ is finitely generated. Thus the left-sided version of Lemma 6.3 shows that $\operatorname{End}_{W}(M)$ is a finitely generated right $R$-module. Moreover, by Proposition $4.10, R$ is a maximal order and so $R=\operatorname{End}_{W}(M)$.

By Lemma 2.13(3), $M^{* *}$ is $g$-divisible with $M^{* *} \subset T_{(g)}$. Since $M^{* *}$ is clearly a finitely generated right $R$-module, the same logic ensures that $F$ is $g$-divisible, ${ }_{F} M^{* *}$ is finitely generated and $\operatorname{End}_{F}\left(M^{* *}\right)=R$. By Lemma 6.2, $F \supseteq W$ and $F$ is the unique maximal order containing and equivalent to $W$. This automatically ensures that $V=F \cap T$ is maximal among $T$-orders containing and equivalent to $W$. Clearly $V$ is also $g$-divisible.

It remains to find the ideal $K$. By Proposition 2.9, both $W$ and $F$ are noetherian. By Proposition 4.10 and Lemma 4.11(1), $\operatorname{GKdim}_{R}\left(M^{* *} / M\right) \leq \operatorname{GKdim}(R)-2=1$. Since $M$ is $g$-divisible, $X=M^{* *} / M$ is $g$-torsionfree and so, by Lemma $2.14, X$ is a finitely generated right $\mathbb{k}[g]$-module. Since $M \subseteq M^{* *} \subset T_{(g)}$ the action of $g$ is central on $X$ and so $X$ is also a finitely generated left $\mathbb{k}[g]$-module. Now, it is routine to check that $M^{* *}$ and hence $X$ are left $W$-modules, while $\mathbb{k}[g] \subseteq W$ since $W$ is $g$-divisible. Thus, $X$ and hence $M^{* *}$ are finitely generated left $W$-modules. Moreover, $\operatorname{GKdim}_{W}(X) \leq \operatorname{GKdim}_{\mathbb{k}[g]}(X) \leq 1$ and so, by [Krause and Lenagan 1985, Lemma 5.3], $I=\ell-\operatorname{ann}_{W}(X)$ satisfies $\operatorname{GKdim}(W / I) \leq 1$.

Now consider $F$. First,

$$
(I F) M \subseteq I F M^{* *} \subseteq I M^{* *} \subseteq M
$$

and hence $I F=I \subseteq W$. Thus $F$ is a finitely generated right $W$-module and (on the left) $\operatorname{GKdim}_{W}(F / W) \leq \operatorname{GKdim}(W / I) \leq 1$. On the other hand, as ${ }_{W} M^{* *}$ is finitely generated, Lemma 6.3 implies that $F=\operatorname{End}_{R}\left(M^{* *}\right)$ is a finitely generated left $W$ module. Thus, by [ibid., Lemma 5.3], again, the right annihilator $I^{\prime}=\mathrm{r}$-ann $W(F / W)$ satisfies GKdim $W / I^{\prime} \leq 1$. Thus $K=I^{\prime} I$ is an ideal of both $F$ and $W$. By the symmetry of the GK-dimension of bimodules finitely generated on both sides [ibid., Corollary 5.4] and the exactness of the GK-dimension [ibid., Theorem 6.14], $\operatorname{GKdim}(F / K) \leq 1$.

Pairs of algebras $(V, F)$ satisfying the conclusions of the proposition will appear multiple times in this paper and so we turn those properties into a definition. For a case when $F \neq V$, see Proposition 10.3.

Definition 6.5. A pair $(V, F)$ is called a maximal order pair if

(1) $F$ and $V$ are $g$-divisible, cg algebras with $V \subseteq F \subseteq T_{(g)}$ and $V \subseteq T$;

(2) $F$ is a maximal order in $Q_{\mathrm{gr}}(F)=Q_{\mathrm{gr}}(T)$ and $V=F \cap T$ is a maximal $T$-order;

(3) there is an ideal $K$ of $F$ with $K \subseteq V$ and GKdim $F / K \leq 1$. 
The next result illustrates the significance of Proposition 6.4 to the structure of maximal $T$-orders.

Corollary 6.6. Let $U \subseteq T$ be a g-divisible cg maximal $T$-order.

(1) There exists an effective divisor $\boldsymbol{d}$ on $E$, with $\operatorname{deg} \boldsymbol{d}<\mu$, and a g-divisible $(U, T(\boldsymbol{d})$ )-module $M \subseteq T$ with $M T=T$ that is finitely generated as both a left $U$-module and a right $T(\boldsymbol{d})$-module. Moreover, $U=\operatorname{End}_{T(\boldsymbol{d})}(M)$ and $T(\boldsymbol{d})=\operatorname{End}_{U}(M)$.

(2) $\left(U, F=\operatorname{End}_{R}\left(M^{* *}\right)\right)$ is a maximal order pair; in particular, if $U$ is a maximal order then $U=F$.

(3) Suppose that every ideal I of $T(\boldsymbol{d})$ satisfying $\operatorname{GKdim}(T(\boldsymbol{d}) / I)=1$ satisfies GKdim $T / I T \leq 1$ (in particular, this holds if $T(\boldsymbol{d})$ has no such ideals $I$ ). Then $U=F$ is a maximal order.

Proof. (1) By Theorem 5.24, there is an effective divisor $\boldsymbol{d}$ with $\operatorname{deg} \boldsymbol{d}<\mu$ so that

$$
U \subseteq V=\operatorname{End}_{T(d)}(M) \subseteq T,
$$

where $M=\widehat{U T(\boldsymbol{d})}$ is a finitely generated $g$-divisible graded right $T(\boldsymbol{d})$-module with $M T=T$. By Theorem 5.24 again, $V$ and $U$ are equivalent orders. Since $U$ is a maximal $T$-order, this forces $U=V$. Finally, $T(\boldsymbol{d})=\operatorname{End}_{U}(M)$ by Proposition 6.4.

(2) As $U=V$, this is a restatement of Proposition 6.4(2).

(3) Just as in the proof of Proposition 6.4, $J=\mathrm{r}-\mathrm{ann}_{R} M^{* *} / M$ is an ideal of $R$ with $\operatorname{GKdim}(R / J) \leq 1$. Note that since $M$ is $g$-divisible, either $M=M^{* *}$ and $J=R$, or else $\operatorname{GKdim}(R / J)=1$.

In either case, the hypotheses imply that $\operatorname{GKdim} T / J T \leq 1$. Now $M^{* *} J T \subseteq$ $M T=T$. Thus

$$
\operatorname{GKdim}(\alpha T+T) / T \leq \mathrm{GKdim} T / J T \leq 1
$$

for any $\alpha \in M^{* *}$. By Proposition 4.10 and Lemma 4.11(1), this implies that $M^{* *} \subseteq T$. This in turn implies that $M^{* *} T=T$ and hence that $F \subseteq T$. Since $U$ is a maximal $T$-order, $U=F$ is a maximal order.

We now turn to the second main aim of this section, which is to describe the structure of $\bar{U}$ for suitable endomorphism rings $U=\operatorname{End}_{T(d)}(M)$. The importance of this result is that the pleasant properties of $\bar{U}$ can be pulled back to $U$.

Theorem 6.7. Let $\boldsymbol{d}$ be an effective divisor on $E$ with $\operatorname{deg} \boldsymbol{d}<\mu$, and let $R=T(\boldsymbol{d})$. Let $M$ be a finitely generated $g$-divisible graded right $R$-module with $R \subseteq M \subseteq T$. Let $U=\operatorname{End}_{R}(M)$ and $F=\operatorname{End}_{R}\left(M^{* *}\right)$. Then there is an effective divisor $\boldsymbol{y}$ on $E$ so that

$$
\bar{F} \doteq \bar{U} \doteq \operatorname{End}_{\bar{R}}(\bar{M}) \doteq B(E, M(-\boldsymbol{x}), \tau) \quad \text { for } \boldsymbol{x}=\boldsymbol{d}-\boldsymbol{y}+\tau^{-1}(\boldsymbol{y}) .
$$

Moreover, if $V=F \cap T$ then $U \subseteq V \subseteq F$ and $(V, F)$ is a maximal order pair. 
The proof of Theorem 6.7 depends on a series of lemmas that will take the rest of this section. Before getting to those results we make some comments and a definition. We first want to regard the ring $F$ from the theorem as a blowup of $T$ at the divisor $\boldsymbol{x}$ on $E$, even if $\boldsymbol{x}$ is not effective. We formalise this as follows.

Definition 6.9. Let $\boldsymbol{x}$ be a (possibly noneffective) divisor on $E$ with $0 \leq \operatorname{deg} \boldsymbol{x}<$ $\mu=\operatorname{deg} M$. We say that a cg algebra $F \subseteq T_{(g)}$ is a blowup of $T$ at $\boldsymbol{x}$ if

(i) $F$ is part of a maximal order pair $(V, F)$ with $Q_{\mathrm{gr}}(F)=Q_{\mathrm{gr}}(T)$; and

(ii) $\bar{F} \doteq B(E, M(-x), \tau)$.

Remarks 6.10. (i) The reader should regard this definition of a blowup as temporary in the sense that it will be refined in Definition 7.1 and justified in Remark 7.5. One caveat about the concept is that there may not be a unique blowup of $T$ at the divisor $\boldsymbol{x}$; in the context of Theorem 6.7 there may be different $R$-modules $M$ leading to distinct blowups $F$, which nonetheless have factors $\bar{F}$ which are equal in large degree. See Example 10.4 and Remark 10.7(2).

(ii) It follows easily from Theorem 6.7 that a maximal order pair $(V, F)$ does give a blowup of $T$ at an appropriate (possibly noneffective) divisor $\boldsymbol{x}$. The details are given in Theorem 7.4 which also gives a converse to Theorem 6.7.

(iii) We conjecture that, generically, the blowup $T(\boldsymbol{d})$ will have no sporadic ideals in Theorem 6.7 and so, by Corollary 6.6(3), $U=F$ will then be a maximal order. For an example where this happens see Example 10.4, and, conversely, for an example when $U \neq F$ and $F \nsubseteq T$ see Proposition 10.3.

Notation 6.11. For the rest of the section, we write $N^{*}=\operatorname{Hom}_{U}(N, U)$ provided that the ring $U$ is clear from the context. In particular, given a $g$-divisible left ideal $I$ of $R$, we have $\bar{I}^{*}=\operatorname{Hom}_{\bar{R}}(I / g I, \bar{R})$ while $\overline{I^{*}}=\overline{\operatorname{Hom}_{R}(I, R)}$. Recall from Lemma 4.11 that a $R$-module $M$ is $\alpha$-pure provided $\operatorname{GKdim}(M)=\operatorname{GKdim}(N)=\alpha$ for all nonzero submodules $N \subseteq M$.

The main technical result we will need is the following, showing that "bar and star commute" (up to a finite-dimensional vector space).

Proposition 6.12. Let $R=T(\boldsymbol{d})$ for an effective divisor $\boldsymbol{d}$ with $\operatorname{deg} \boldsymbol{d}<\mu$.

(1) Let I be a proper, g-divisible left ideal of $R$ for which $R / I$ is 2-pure. Then $I^{*} / R$ is a g-torsionfree, 2-pure right module; further, $I^{*} \subseteq T_{(g)}$ and $\overline{I^{*}} \doteq \bar{I}^{*}$.

(2) If $M$ is a finitely generated g-divisible graded right $R$-module with $R \subseteq M \subseteq T$, then $\overline{M^{*}} \doteq \bar{M}^{*}$.

Proof. (1) By Lemma 4.11(2), $I^{*} / R$ is 2-pure. By Lemma 2.12, $I^{*} \subseteq T_{(g)}$ and since $R$ is $g$-divisible, $T_{(g)} / R$ and hence $I^{*} / R$ are $g$-torsionfree. 
From the exact sequence $0 \rightarrow R g \rightarrow R \rightarrow \bar{R} \rightarrow 0$ we obtain the long exact sequence of right $R$-modules

$$
\begin{array}{r}
0 \rightarrow \operatorname{Hom}_{R}(R / I, \bar{R}) \rightarrow \operatorname{Ext}_{R}^{1}(R / I, R g) \rightarrow \operatorname{Ext}_{R}^{1}(R / I, R) \rightarrow \operatorname{Ext}_{R}^{1}(R / I, \bar{R}) \\
\stackrel{\phi}{\rightarrow} \operatorname{Ext}_{R}^{2}(R / I, R g) \stackrel{\psi}{\rightarrow} \operatorname{Ext}_{R}^{2}(R / I, R) \rightarrow \operatorname{Ext}_{R}^{2}(R / I, \bar{R}) \rightarrow \cdots .
\end{array}
$$

By Proposition 4.10, $\bar{R}$ is Auslander-Gorenstein and CM. Thus $N=\operatorname{Ext}_{\bar{R}}^{2}(\bar{R} / \bar{I}, \bar{R})$ has grade $j(N) \geq 2$ and hence $\operatorname{GKdim}(N) \leq 2-2=0$. Therefore, by [RSS 2015, Lemma 7.9], $\operatorname{Ext}_{R}^{2}(R / I, \bar{R})=N$ is finite-dimensional and the map $\psi$ in (6.13) is surjective in large degree. If $E=\operatorname{Ext}_{R}^{2}(R / I, R)$, this says that $\psi: E[-1] \rightarrow E$ is surjective in large degree. Since $\operatorname{dim}_{\mathbb{k}} E_{n}<\infty$ for each $n$, this forces $\operatorname{dim}_{\mathbb{k}} E_{n} \geq$ $\operatorname{dim}_{\mathbb{k}} E_{n+1}$ for all $n \gg 0$ and so $\operatorname{dim}_{\mathbb{k}} E_{n}$ is eventually constant. In turn, this forces $\phi$ to be zero in large degree.

Next, observe that $\operatorname{Hom}(R / I, \bar{R})=0$ since $R / I$ is $g$-torsionfree. Since $\phi$ is zero in high degree, the complex

$$
0 \longrightarrow \operatorname{Ext}_{R}^{1}(R / I, R g) \longrightarrow \operatorname{Ext}_{R}^{1}(R / I, R) \longrightarrow \operatorname{Ext}_{R}^{1}(R / I, \bar{R}) \longrightarrow 0
$$

is exact in high degree. Using [RSS 2015, Lemma 7.9] this can be identified with the complex

$$
0 \longrightarrow\left(I^{*} / R\right)[-1] \stackrel{\alpha}{\longrightarrow} I^{*} / R \longrightarrow \operatorname{Ext}_{\bar{R}}^{1}(\bar{R} / \bar{I}, \bar{R}) \longrightarrow 0,
$$

where $\alpha$ is multiplication by $g$. As $I^{*}$ is $g$-divisible by Lemma 2.12(2), it follows that

$$
\overline{I^{*}} / \bar{R} \cong I^{*} /\left(R+I^{*} g\right)=\operatorname{coker}(\alpha) \doteq \operatorname{Ext}_{\bar{R}}^{1}(\bar{R} / \bar{I}, \bar{R})=\bar{I}^{*} / \bar{R} .
$$

In particular, $\operatorname{dim}_{\mathbb{k}} \overline{I^{*}}=\operatorname{dim}_{\mathbb{k}} \bar{I}^{*}$ for all $n \gg 0$, and as there is an obvious inclusion $\overline{I^{*}} \subseteq \bar{I}^{*}$ we conclude that $\overline{I^{*}} \doteq \bar{I}^{*}$.

(2) Note that $M^{* *} / M$ is a $g$-torsionfree module of GK-dimension 1, as in the proof of Proposition 6.4. By Lemma 2.14, $\operatorname{dim}_{\mathbb{k}}\left(\left(M^{* *} / M\right) \otimes_{R} \bar{R}\right)<\infty$. Thus $\overline{M^{* *}} \doteq \bar{M}$.

Let $J=M^{*}$. Since $J$ is a reflexive left ideal of $R$, the module $R / J$ is 2-pure by Lemmas 4.10(3) and 4.11(3). Thus part (1) applies and shows that $\bar{J}^{*} \doteq \overline{J^{*}}$. Next, $\bar{J} \doteq \bar{J}^{* *}$ by another use of Lemmas 4.10(3) and 4.11(3). Finally, it is easy to see that for any finitely generated graded $\bar{R}$-modules $N$ and $Q$ contained in $Q_{\mathrm{gr}}(\bar{R})$, if $N \doteq Q$ then $N^{*} \doteq Q^{*}$. Putting the pieces above together, we conclude that

$$
\overline{M^{*}}=\bar{J} \doteq \bar{J}^{* *} \doteq\left(\overline{J^{*}}\right)^{*} \doteq \bar{M}^{*} .
$$

The last ingredient we need for the proof of Theorem 6.7 is the following description of the endomorphism ring of a torsion-free rank-one module over a twisted homogeneous coordinate ring. 
Lemma 6.14. Let $B=B(E, \mathscr{L}, \tau)$, where $E$ is a smooth elliptic curve, $\operatorname{deg} \mathscr{L} \geq 1$, and $\tau$ is of infinite order. Let $N$ be a finitely generated, graded right $B$-submodule of $\mathbb{k}(E)\left[t, t^{-1} ; \tau\right]$; by [Artin and Van den Bergh 1990, Theorem 1.3],

$$
N \doteq \bigoplus_{r \geq 0} H^{0}\left(E, \mathcal{O}(\boldsymbol{q}) \otimes \mathscr{L}_{r}\right)
$$

for some divisor q. Let $N^{*}=\operatorname{Hom}_{B}(N, B) \subseteq \mathbb{k}(E)\left[t, t^{-1} ; \tau\right]$. Then:

(1) $\operatorname{End}_{B}(N) \doteq B\left(E, \mathscr{L}\left(\boldsymbol{q}-\tau^{-1}(\boldsymbol{q})\right), \tau\right)$.

(2) $N N^{*} \doteq \operatorname{End}_{B}(N)$.

(3) $N^{*} \doteq \bigoplus_{n \geq 0} H^{0}\left(E, \mathscr{L}_{n} \otimes \mathcal{O}\left(-\tau^{-n}(\boldsymbol{q})\right)\right)$.

Proof. (1) Write $G=\operatorname{End}_{B}(N) \subseteq \mathbb{k}(E)\left[t, t^{-1} ; \tau\right]$ and, for each $n$, let $\mathscr{G}_{n}$ be the subsheaf of the constant sheaf $\mathbb{k}(E)$ generated by $G_{n} \subseteq \mathbb{k}(E)$. Let $\mathcal{N}_{n}=\mathbb{O}(\boldsymbol{q}) \otimes \mathscr{L}_{n}$; thus $N_{n}=H^{0}\left(E, \mathcal{N}_{n}\right)$, and $N_{n}$ generates the sheaf $\mathcal{N}_{n}$, for $n \gg 0$, say $n \geq n_{0}$.

For $n \geq n_{0}$ and $r \geq 0$, the equation $G_{r} N_{n} \subseteq N_{n+r}$ forces $\mathscr{G}_{r} \mathcal{N}_{n}^{\tau^{r}} \subseteq \mathcal{N}_{n+r}$ and thus

$$
\mathscr{G}_{r} \otimes\left(\mathcal{O}(\boldsymbol{q}) \otimes \mathscr{L}_{n}\right)^{\tau^{r}} \subseteq \mathcal{O}(\boldsymbol{q}) \otimes \mathscr{L}_{n+r} .
$$

Equivalently,

$$
\mathscr{G}_{r} \subseteq \mathscr{L}_{r}\left(\boldsymbol{q}-\tau^{-r}(\boldsymbol{q})\right)=\left(\mathscr{L}\left(\boldsymbol{q}-\tau^{-1}(\boldsymbol{q})\right)\right)_{r}
$$

This shows that

$$
G \subseteq B\left(E, \mathscr{L}\left(\boldsymbol{q}-\tau^{-1}(\boldsymbol{q})\right), \tau\right) .
$$

Reversing this calculation shows that

$$
\left(\mathscr{L}\left(\boldsymbol{q}-\tau^{-1}(\boldsymbol{q})\right)\right)_{r} \mathcal{N}_{n}^{\tau^{r}} \subseteq \mathcal{N}_{n+r}
$$

for $r, n \geq 0$ and taking sections for $n \geq n_{0}$ shows that

$$
B\left(E, \mathscr{L}\left(\boldsymbol{q}-\tau^{-1}(\boldsymbol{q})\right), \tau\right) \subseteq \operatorname{End}_{B}\left(N_{\geq n_{0}}\right) .
$$

To complete the proof we need to prove that $G \doteq \operatorname{End}_{B}\left(N_{\geq n_{0}}\right)$. This follows by [Rogalski 2011, Lemma 2.2(2)] and [Artin and Zhang 1994, Proposition 3.5] or by a routine computation.

(2) Clearly $N N^{*}$ is an ideal of $\operatorname{End}_{B}(N)$. However, by Lemma 2.7(2), $\operatorname{End}_{B}(N)$ is just infinite, and so $N N^{*} \doteq \operatorname{End}_{B}(N)$.

(3) The proof is similar to that of (1) and, as it will not be used in the paper, is left to the reader.

Proof of Theorem 6.7. We first check that $\bar{F} \doteq \bar{U}$. By Proposition 6.4 there exists an ideal $K$ of $F$ contained in $U$ and satisfying $\operatorname{GKdim}(F / K) \leq 1$. In particular, $\operatorname{GKdim}(F / U) \leq 1$. By Lemma 2.12(3), $U$ is $g$-divisible, and so $N=F / U$ is $g$-torsionfree. It follows from Lemma 2.14 that $\operatorname{GKdim}(\bar{F} / \bar{U})=0$, and so $\bar{U} \doteq \bar{F}$. 
Now it is obvious that $U \supseteq M M^{*}$. Thus, using Proposition 6.12(2),

$$
\bar{U} \supseteq(\bar{M})\left(\overline{M^{*}}\right) \doteq(\bar{M})\left(\bar{M}^{*}\right) .
$$

Conversely, by Lemma 2.12(3), $\bar{U}=\overline{\operatorname{End}_{R}(M)} \subseteq \operatorname{End}_{\bar{R}}(\bar{M})$. We also have $\bar{R}=B(E, M(-d), \tau)$. Applying Lemma 6.14 to $\mathscr{L}=M(-\boldsymbol{d})$ and $N=\bar{M}$ gives

$$
(\bar{M})\left(\bar{M}^{*}\right) \doteq \operatorname{End}_{\bar{R}}(\bar{M}) \doteq B(E, M(-x), \tau),
$$

where, in the notation of that lemma, $\boldsymbol{y}=\boldsymbol{q}$ and $\boldsymbol{x}=\boldsymbol{d}-\boldsymbol{y}+\tau^{-1}(\boldsymbol{y})$. That $\boldsymbol{y}$ is effective follows from $\bar{R} \subseteq \bar{M}$. Combining the last two displayed equations gives (6.8).

Since $R \subseteq M$, necessarily $M T=T$. Thus the second paragraph of the theorem is just a restatement of Proposition 6.4.

\section{The structure of $g$-divisible orders}

In this section we first refine the results from the last two sections to give strong structural results for a $g$-divisible maximal $T$-order $U$ (see Theorem 7.4). Then we use these results to analyse both arbitrary $g$-divisible orders and ungraded subalgebras of $D=D_{\mathrm{gr}}(T)$ (see Corollaries 7.6 and 7.10, respectively). In particular, we show that $U$ is part of a maximal order pair $(U, F)$ for which $F$ is a blowup of $T$ at a (possibly noneffective) divisor $\boldsymbol{x}=\boldsymbol{d}-\boldsymbol{y}+\tau^{-1}(\boldsymbol{y})$ in the sense of Definition 6.9. Here, the divisor $\boldsymbol{y}$ can have arbitrarily high degree but is not arbitrary, as we first explain.

Definition 7.1. Let $\boldsymbol{x}$ be a divisor on $E$. For each $\tau$-orbit $\mathbb{O}$ in $E$ pick $p=p_{0} \in \mathbb{O}$ such that $\left.\boldsymbol{x}\right|_{\mathbb{O}}=\sum_{i=0}^{k} x_{i} p_{i}$, where $p_{i}=\tau^{-i}(p)$. Then $\boldsymbol{x}$ is called a virtually effective divisor if for each orbit $\mathbb{O}$ and all $j \in \mathbb{Z}$ the divisor $\boldsymbol{x}$ satisfies

$$
\sum_{i \leq j} x_{i} \geq 0 \quad \text { and } \quad \sum_{i \geq j} x_{i} \geq 0
$$

If $F$ is a blowup of $T$ at a virtually effective divisor $x$ then $F$ is called a virtual blowup of $T$.

The relevance of this condition is shown by the next result, in which the notation $\boldsymbol{u}_{k}$ for a divisor $\boldsymbol{u}$ comes from Notation 5.2.

Proposition 7.3. (1) The divisor $\boldsymbol{x}$ in Theorem 6.7 is virtually effective.

(2) A divisor $\boldsymbol{x}$ is virtually effective if and only if $\boldsymbol{x}$ can be written as

$$
\boldsymbol{x}=\boldsymbol{u}-\boldsymbol{v}+\tau^{-1}(\boldsymbol{v})
$$

where $\boldsymbol{u}$ is an effective divisor supported on distinct $\tau$-orbits and $\boldsymbol{v}$ is an effective divisor such that $0 \leq \boldsymbol{v} \leq \boldsymbol{u}_{k}$ for some $k$. 
Proof. (1) By Theorem 6.7, $\bar{F} \doteq B(E, \mathcal{N}, \tau)$, where $\mathcal{N}=\mu(-x)$. Since $\bar{F} \doteq \bar{U} \subseteq$ $\bar{T}=B(E, M, \tau)$, we must have $\mathcal{N}_{n} \subseteq M_{n}$ for $n \gg 0$. Now compare this with the computations in the proof of Corollary 5.3. In the notation of that proof, $y=\mathbb{O}_{E}$ and hence $\boldsymbol{y}=0$. Therefore, as is explained in the proof of (5.11), this forces (7.2) to hold.

(2) It is enough to prove this in the case that $\boldsymbol{x}$ is supported on a single $\tau$-orbit $\mathbb{O}$ in $E$.

$(\Rightarrow)$ As in Definition 7.1, write $\boldsymbol{x}=\sum_{i=0}^{k} x_{i} p_{i}$ for a suitable point $p_{0} \in \mathbb{O}$. Set $e=\sum_{i \in \mathbb{Z}} x_{i}$ and $\boldsymbol{u}=e p$. For $j \in \mathbb{N}$, let $v_{j}=\sum_{i \geq j+1} x_{i}$ and put $\boldsymbol{v}=\sum_{j \geq 0} v_{j} p_{j}$.

By (7.2), $v$ is effective. Also, since $\sum_{i \leq j} x_{i} \geq 0$ for all $j$, we have

$$
v_{j}=e-\sum_{i \leq j} x_{i} \leq e \quad \text { for } 0 \leq j \leq k-1,
$$

while $v_{j}=0$ for $j \geq k$. Therefore, $0 \leq \boldsymbol{v} \leq \boldsymbol{u}_{k}=\sum_{i=0}^{k-1} e p_{i}$. Finally,

$$
\begin{aligned}
\boldsymbol{u}-\boldsymbol{v}+\tau^{-1}(\boldsymbol{v}) & =e p_{0}-\sum_{j \geq 0}\left(\sum_{i \geq j+1} x_{i}\right) p_{j}+\sum_{j \geq 0}\left(\sum_{i \geq j+1} x_{i}\right) p_{j+1} \\
& =e p_{0}-\left(\left(\sum_{i \geq 1} x_{i}\right) p_{0}+\sum_{j \geq 1}\left(\sum_{i \geq j+1} x_{i}\right) p_{j}\right)+\sum_{j \geq 1}\left(\sum_{i \geq j} x_{i}\right) p_{j} \\
& =\sum x_{i} p_{i}=\boldsymbol{x} .
\end{aligned}
$$

$(\Leftarrow)$ Although this is similar to part $(1)$, it seems easiest to give a direct proof.

Write $\boldsymbol{u}=e p=e p_{0}$ and $\boldsymbol{v}=\sum v_{i} p_{i}$ for some point $p$ and some $v_{j} \geq 0$. By definition, $\boldsymbol{u}_{k}=\sum_{i=0}^{k-1} e p_{i}$, and so, by our assumptions, $0 \leq v_{i} \leq e$ for $0 \leq i \leq k-1$, and $v_{i}=0$ for all other $i$. Therefore,

$$
\boldsymbol{x}=\boldsymbol{u}-\boldsymbol{v}+\tau^{-1}(\boldsymbol{v})=\left(e-v_{0}\right) p_{0}+\sum_{i \geq 1}\left(v_{i-1}-v_{i}\right) p_{i} .
$$

If $j \leq-1$ then $x_{j}=0$ and $\sum_{i \leq j} x_{i}=0$. If $j \geq 0$, then $\sum_{i \leq j} x_{i}=e-v_{j} \geq 0$. Similarly, if $j \leq 0$ then $\sum_{i \geq j} x_{i}=e \geq 0$, while if $j \geq 1$ then

$$
\sum_{i \geq j} x_{i}=\sum_{i=j}^{k}\left(v_{i-1}-v_{i}\right)=v_{j-1}-v_{k}=v_{j-1} \geq 0 .
$$

Thus (7.2) is satisfied.

We are now ready to state our main result on the structure of $g$-divisible maximal $T$-orders. 
Theorem 7.4. (1) Let $V \subseteq T$ be a g-divisible cg maximal $T$-order. Then the following hold:

(a) There is a maximal order $F \supseteq V$ such that $(V, F)$ is a maximal order pair.

(b) $F$ is a virtual blowup of $T$ at a virtually effective divisor $\boldsymbol{x}=\boldsymbol{u}-\boldsymbol{v}+\tau^{-1}(\boldsymbol{v})$ satisfying $0 \leq \operatorname{deg} \boldsymbol{x}<\mu$.

(c) $\bar{V} \doteq \bar{F} \doteq B(E, M(-x), \tau)$.

(2) If $U \subseteq T$ is any g-divisible cg subalgebra with $Q_{\mathrm{gr}}(U)=Q_{\mathrm{gr}}(T)$, there exists a maximal order pair $(V, F)$ as in (1) such that $U$ is contained in and equivalent to $V$.

(3) Conversely, let $\boldsymbol{x}$ be a virtually effective divisor with $\operatorname{deg} \boldsymbol{x}<\mu$. Then there exists a blowup $F$ of $T$ at $\boldsymbol{x}$.

Proof. (1) By definition, $Q_{\mathrm{gr}}(V)=Q_{\mathrm{gr}}(T)$. Now combine Corollary 6.6(1-2), Theorem 6.7 and Proposition 7.3.

(2) By Theorem 5.24, $U$ is contained in and equivalent to some $\operatorname{End}_{T(d)}(M)$ which, in turn, is contained in and equivalent to a maximal $T$-order by Proposition 6.4.

(3) Write $\boldsymbol{x}=\boldsymbol{u}-\boldsymbol{v}+\tau^{-1}(\boldsymbol{v})$, where $\boldsymbol{u}, \boldsymbol{v}, k$ are defined by applying Proposition 7.3 to $\boldsymbol{x}$. By [RSS 2015, Lemma 5.10], there is a $g$-divisible finitely generated right $T(\boldsymbol{u})$-module $M$ with $T(\boldsymbol{u}) \subseteq M \subseteq M T=T$ so that

$$
\bar{M} \doteq \bigoplus_{n} H^{0}\left(E, \mu_{n}\left(-\boldsymbol{u}_{n}+\boldsymbol{v}\right)\right) .
$$

Let $F=\operatorname{End}_{R}\left(M^{* *}\right) \supseteq U=\operatorname{End}_{R}(M)$. By Theorem 6.7 and Lemma 6.14(1-2), we have

$$
\bar{F} \doteq \bar{U} \doteq \bar{M}(\bar{M})^{*} \doteq B(E, M(-x), \tau),
$$

and $(F \cap T, F)$ is a maximal order pair.

Remark 7.5. We should explain why $F$ is called a virtual blowup of $T$ at $x$ both in this theorem and in Definition 7.1. When $\boldsymbol{x}$ is effective this is amply justified in [Rogalski 2011] and, in that case, $T(\boldsymbol{x})$ satisfies many of the basic properties of a commutative blowup; in particular, it agrees with Van den Bergh's more categorical blowup [2001]. For noneffective $\boldsymbol{x}$ there are several reasons why the notation is reasonable.

(1) As we have shown repeatedly in this paper, the factor $\bar{U}$ of a $g$-divisible algebra $U$ controls much of $U$ 's behaviour and so Theorem 7.4(1c) shows that $F$ will have many of the basic properties of a blowup at an effective divisor.

(2) This is also supported by the fact that, by Theorem 5.24, $F$ and $T(\boldsymbol{u})$ are equivalent maximal orders and, again, many properties pass through such a Morita context. 
(3) Finally, in the commutative case virtual blowups are blowups, both because virtually effective divisors are then effective and because equivalent maximal orders are then equal.

Theorem 7.4 can be easily used to describe arbitrary $g$-divisible subalgebras of $T$. We recall that the idealiser of a left ideal $L$ in a $\operatorname{ring} A$ is the subring $\llbracket(L)=\{\theta \in A: L \theta \subseteq L\}$.

Corollary 7.6. Let $U \subseteq T$ be a g-divisible subalgebra with $Q_{\mathrm{gr}}(U)=Q_{\mathrm{gr}}(T)$. Then $U$ is an iterated subidealiser inside a virtual blowup of $T$. More precisely, we have the following chain of rings:

(1) There is a virtually effective divisor $\boldsymbol{x}=\boldsymbol{u}-\boldsymbol{v}+\tau^{-1}(\boldsymbol{v})$ with $\operatorname{deg}(\boldsymbol{x})<\mu$ and a blowup $F$ of $T$ at $\boldsymbol{x}$ such that $V=F \cap T$ contains and is equivalent to $U$, while $(V, F)$ is a maximal order pair.

(2) There exist a g-divisible algebra $W$ with $U \subseteq W \subseteq V$ such that $U$ is a right subidealiser inside $W$ and $W$ is a left subidealiser inside $V$. In more detail,

(a) There exists a graded g-divisible left ideal $L$ of $V$ such either $L=V$ or else $V / L$ is 2-pure, and a g-divisible ideal $K$ of $X=\llbracket(L)$ such that $K \subseteq W \subseteq X$ and $\operatorname{GKdim}_{X}(X / K) \leq 1$

(b) $V$ is a finitely generated left $W$-module, while $X / K$ is a finitely generated $\mathbb{k}[g]$-module and so $X$ is finitely generated over $W$ on both sides;

(c) the properties given for $W \subseteq V$ also hold for the pair $U \subseteq W$, but with left and right interchanged.

Proof. (1) Use Theorem 7.4(1-2).

(2) By (1), $a V b \subseteq U$ for some $a, b \in U \backslash\{0\}$. Set $W^{\prime}=U+V b$ and $W=\widehat{W^{\prime}}$. By Lemma 2.13(1), $a W=\widehat{a W^{\prime}} \subseteq \widehat{U}=U$. By Proposition 2.9, $W$ is noetherian and so (modulo a shift) $V \cong V b$ is a finitely generated left $W$-module. Similarly, $W$ is a finitely generated right $U$-module. We will now just prove parts (2a) and (2b), leaving the reader to check that the same argument does indeed work for the pair $(U, W)$.

Write $V=\sum_{i=1}^{v} W e_{i}$ for some $e_{i}$. Then the right annihilator

$$
K=\operatorname{rann}_{W}(V / W)=\bigcap \mathrm{r}-\operatorname{ann}\left(e_{i}\right)
$$

is nonzero. Let $L / K$ be the largest left $V$-submodule of $V / K$ with $\operatorname{GKdim}(L / K) \leq 1$. Then either $L=V$, or else $V / L$ is 2-pure. For $a \in W$, the module $(L a+K) / K$ is a homomorphic image of $L a / K a$ and hence of $L / K$. Thus $\operatorname{GKdim}((L a+K) / K) \leq 1$ and $L a \subseteq L$; in other words, $L$ is still a $(V, W)$-bimodule.

As $W=\widehat{W}$, it is routine to see that $K$ is $g$-divisible, but since we use the argument several times we give the details. So, suppose that $\theta g \in K$ for some $\theta \in V$. Then $(V \theta) g \subseteq \widehat{W}=W$, whence $V \theta \subseteq W$ and $\theta \in K$, as required. It follows that $L / K$ is $g$-torsionfree and so, by Lemma $2.14, L / K$ is a finitely 
generated right $\mathbb{k}[g]$-module. Thus, by [Krause and Lenagan 1985, Lemma 5.3], $I=\ell-\operatorname{ann}_{V}(L / K)$ satisfies $\operatorname{GKdim}_{V}(V / I)=\operatorname{GKdim}(L / K) \leq 1$. Again, $I$ is $g$ divisible. Also, if $\theta \in V$ has $\theta g \in L$ then $(I \theta) g \subseteq K$ and so $I \theta \subseteq K$. Hence $\operatorname{GKdim}(V \theta+K) / K \leq \operatorname{GKdim}(V / I) \leq 1$ and $\theta \in L$. So $L$ is also $g$-divisible.

Finally, let $X=\rrbracket_{V}(L)=\{x \in V: L x \subseteq L\}$. As usual, $X$ is $g$-divisible. Clearly $I L$ is an ideal of $X$, and since $I$ and $L$ are $g$-divisible, $\operatorname{GKdim}(X / I L) \leq 1$, by Lemma 2.15(4). Since $X \supset K \supseteq I L$, it follows that $\mathrm{GKdim} X / K \leq 1$. Finally, since $X / K$ is $g$-torsionfree of GK-dimension 1 , it must be a finitely generated $\mathbb{k}[g]$ module by Lemma 2.14; in particular, $X / W$ and hence $X$ are finitely generated as right $W$-modules.

There is a close correspondence between subalgebras $A$ of the function skewfield $D=D_{\mathrm{gr}}(T)$ and $g$-divisible subalgebras of $T_{(g)}$, and so we end the section by studying the consequences of our earlier results for such an algebra $A$.

For a cg subalgebra $R \subseteq T_{(g)}$ with $g \in R$, define

$$
R^{\circ}=R\left[g^{-1}\right]_{0}=\bigcup_{n \geq 0} R_{n} g^{-n} \subseteq D=D_{\mathrm{gr}}(T) .
$$

Conversely, given an algebra $A \subseteq T^{\circ}$, define

$$
\Omega A=\bigoplus_{m \geq 0}(\Omega A)_{m} \quad \text { for }(\Omega A)_{m}=\left\{a \in T_{m}: a g^{-m} \in A\right\} .
$$

Clearly $\Omega A$ is $g$-divisible with $(\Omega A)^{\circ}=A$ and, if $R \subseteq T$, then $\Omega\left(R^{\circ}\right)=\widehat{R}$; thus we obtain a one-to-one correspondence between cg $g$-divisible subalgebras of $T$ and subalgebras of $T^{\circ}$.

Given a left ideal $I$ of $R$ or a left ideal $J$ of $A$ we define $I^{\circ}$ and $\Omega J$ by the same formulæ. If $R$ is $g$-divisible, the map $I \mapsto I^{\circ}$ gives a one-to-one correspondence between $g$-divisible left ideals of $R$ and left ideals of $R^{\circ}$, with analogous results for two-sided ideals (see [Artin et al. 1991, Proposition 7.5]).

An algebra $A \subseteq T^{\circ}$ is filtered by $A=\bigcup \Gamma^{n} A$ for $\Gamma^{n} A=(\Omega A)_{n} g^{-n}$. By [RSS 2014, Lemmas 2.1 and 2.2],

$$
\operatorname{gr}_{\Gamma} A=\bigoplus \Gamma^{n} A / \Gamma^{n-1} A \cong \Omega A / g \Omega A,
$$

where the isomorphism is induced by the map

$$
\Gamma^{n} A \backslash \Gamma^{n-1} A \rightarrow \Omega A, \quad x=r g^{-n} \mapsto r .
$$

Lemma 7.8. Let $A, A^{\prime}$ be orders in $T^{\circ}$. Then $A$ and $A^{\prime}$ are equivalent orders if and only if $\Omega A$ and $\Omega A^{\prime}$ are equivalent orders in $Q_{\mathrm{gr}}(T)$.

Proof. Let $0 \neq a \in \Gamma_{m} A^{\prime}$ and $0 \neq b \in \Gamma_{n} A^{\prime}$. To prove the lemma, it suffices to show that $a A b \subseteq A^{\prime}$ if and only if $a g^{m}(\Omega A) b g^{n} \subseteq \Omega A^{\prime}$. However, if $0 \neq \alpha \in \Omega A$, write 
$\alpha=x g^{k}$ for some $k$ and $x \in A$. Then

$$
a x b \in A^{\prime} \Longleftrightarrow a x b \in \Gamma_{m+n+k} A^{\prime} \Longleftrightarrow a g^{m}\left(x g^{k}\right) b g^{n} \in \Omega A^{\prime},
$$

as desired.

Corollary 7.9. A subalgebra $A \subseteq T$ is a maximal $T^{\circ}$-order if and only if $\Omega(A)$ is a maximal $T$-order.

By [RSS 2014, Theorem 1.1], every subalgebra of $T^{\circ}$ is finitely generated and noetherian; these subalgebras thus give a rich supply of noetherian domains of GK-dimension 2. Our earlier results about cg maximal $T$-orders translate easily to results about maximal $T^{\circ}$-orders. An ideal $I$ of a $\mathbb{k}$-algebra $A$ is called cofinite if $\operatorname{dim}_{\mathbb{k}}(A / I)<\infty$.

Corollary 7.10. Let A be a subalgebra of $T^{\circ}$ with $Q(A)=Q\left(T^{\circ}\right)$.

(1) There exists a maximal order pair $(V, F)$, where $F$ is a blowup of $T$ at some virtually effective divisor $\boldsymbol{x}$, such that $A$ is contained in and equivalent to the maximal $T$-order $V^{\circ}$.

(2) In part (1), $F^{\circ}$ is a maximal order in $Q\left(T^{\circ}\right)=D_{\mathrm{gr}}(T)$.

(3) The algebras $V^{\circ}$ and $F^{\circ}$ have a cofinite ideal $K^{\circ}$ in common. Also, we have $\operatorname{gr}_{\Gamma} V \doteq B(E, M(-x), \tau)$.

(4) Suppose that all nonzero ideals I of $T(\boldsymbol{d})^{\circ}$ generate cofinite right ideals of $T^{\circ}$ (in particular, this happens if $T(\boldsymbol{d})^{\circ}$ is simple) and that $A$ is a maximal $T^{\circ}$-order. Then $A$ is a maximal order.

Proof. (1) By Theorem 7.4(2), $\Omega A$ is contained in and equivalent to some such $V$. Now use Lemma 7.8 and Corollary 7.9.

(2) Since $F$ need not be contained in $T$, this does not follow directly from the above discussion. However, it does follow from Lemma 6.2 combined with the fact that, in the notation of Corollary 6.6,

$$
F^{\circ}=\operatorname{End}_{T(\boldsymbol{d})^{\circ}}\left(\left(M^{* *}\right)^{\circ}\right)=\operatorname{End}_{T(\boldsymbol{d})^{\circ}}\left(\left(M^{\circ}\right)^{* *}\right) .
$$

(3) By definition and Lemma 2.14, $V$ and $F$ have an ideal $K$ in common such that $F / K$ is finitely generated as a $\mathbb{k}[g]$-module. Consequently $F^{\circ} / K^{\circ}$ and $V^{\circ} / K^{\circ}$ are finite-dimensional. The final assertion follows from Theorem 7.4(1c).

(4) Use Corollary 6.6(3).

We also have a converse to Corollary 7.10(3).

Corollary 7.11. Let $\boldsymbol{x}$ be a virtually effective divisor on $E$ with $\operatorname{deg} \boldsymbol{x}<\mu$. Then there exists a maximal $T^{\circ}$-order $A$ with $\operatorname{gr}_{\Gamma} A \doteq B(E, M(-x), \tau)$. 
Proof. Let $U$ be the $g$-divisible maximal $T$-order given by Theorem 7.4(3); thus $\bar{U} \doteq B(E, M(-\boldsymbol{x}), \tau)$ by part (1c) of that result. By (7.7), $A=U^{\circ}$ satisfies the conclusion of this corollary.

Del Pezzo surfaces. The blowup of $T$ at $\leq 8$ points on $E$ can be thought of as a noncommutative del Pezzo surface. More carefully, it should be thought of as the anticanonical ring of a noncommutative del Pezzo surface; this corresponds to the fact that the central element $g$ is in degree 1. Let $U$ be a blowup of $T$ at a virtually effective divisor $\boldsymbol{d}^{\prime}$ of degree $\leq 8$. By analogy, we should think of $U$ as a (new type of) noncommutative del Pezzo surface, and the localisation $U^{\circ}$ as a particular kind of noncommutative affine surface. Corollary 7.10(3) can then be reinterpreted as saying that any maximal order $A \subseteq T^{\circ}$ is the coordinate ring of just such a noncommutative affine surface.

In [Etingof and Ginzburg 2010], the authors study noncommutative affine surfaces which are deformations of the commutative symplectic affine surfaces obtained from removing an anticanonical divisor from $\mathbb{P}^{2}$. These surfaces are related to ours but not the same; for example, we consider $A=T^{\circ} \cong T /(g-1)$, but the algebra $A^{\prime}=S /(g-1)$ is considered in [ibid.]. The algebra $A^{\prime}$ is a rank $3 A$-module, so "Spec $A^{\prime \prime}$ " is a triple cover of "Spec $A$ " (inasmuch as these terms make sense in a noncommutative context).

\section{Sporadic ideals and $g$-divisible hulls}

One of the main results in [Rogalski 2011] showed that the algebras considered there have minimal sporadic ideals, in a sense we define momentarily. In this section we show that, under minor assumptions, this generalises to cg subalgebras $U \subseteq T$ with $g \in U$ (see Corollary 8.8 for the precise statement). The significance of this result is that it provides a tight connection between the algebra $U$ and its $g$-divisible hull $\widehat{U}$ and provides the final step in the proof of Theorem 1.2, that maximal orders are noetherian blowups of $T$ (see Theorem 8.11).

Recall that a graded ideal $I$ of a cg graded algebra $R$ is called sporadic if $\operatorname{GKdim}(R / I)=1$.

Definition 8.1. An ideal $I$ of a cg algebra $R$ is called a minimal sporadic ideal if $\operatorname{GKdim}(R / I) \leq 1$ and, for all sporadic ideals $J$, we have $\operatorname{dim}_{\mathbb{k}} I /(J \cap I)<\infty$.

Note that one can make the minimal sporadic ideal $I$ unique by demanding that it be saturated, but we will not do so since this causes extra complications.

Beginning in this section, we need to strengthen our hypothesis on the ring $T$.

Assumption 8.2. In addition to Assumption 2.1, we assume that $T$ has a minimal 
sporadic ideal and that there exists an uncountable algebraically closed field extension $K \supseteq \mathbb{k}$ such that, in the notation of [RSS 2015, Definition 7.2], $\operatorname{Div}\left(T \otimes_{\mathbb{k}} K\right)$ is countable.

We emphasise that, by [RSS 2015, Theorem 8.8 and Proposition 8.7], these extra assumptions do hold both for the algebras $T$ from Examples 2.2(1-2) and for their blowups $T(\boldsymbol{d})$ at effective divisors $\boldsymbol{d}$ with $\operatorname{deg} \boldsymbol{d}<\mu$.

For the rest of this section we assume that our algebras $T$ satisfy Assumptions 2.1 and 8.2. We do not know if Assumption 8.2 holds for Stephenson's algebras from Examples 2.2(3). By a routine exercise, Examples 2.2(4) does not have a minimal sporadic ideal, so Assumption 8.2 is strictly stronger than Assumption 2.1.

As noted above, the blowups $T(\boldsymbol{d})$ with $\operatorname{deg} \boldsymbol{d}<\mu$ have a minimal sporadic ideal, and the first goal of this section is to extend this to more general subalgebras of $T_{(g)}$. We start with the case of $g$-divisible algebras.

Lemma 8.3. Let $(V, F)$ be a maximal order pair, in the sense of Definition 6.5. Then both $F$ and $V$ have a minimal sporadic ideal.

Proof. By Corollary 6.6, there exists an effective divisor $\boldsymbol{d}$ with $\operatorname{deg} \boldsymbol{d}<\mu$ and a right $R$-module $M \supseteq R$, where $R=T(\boldsymbol{d})$, such that

$$
F=\operatorname{End}_{R}\left(M^{* *}\right) \supseteq F \cap T=V=\operatorname{End}_{R}(M) .
$$

We will use a minimal sporadic ideal of $R$ to construct such an ideal for $F$ and for $V$.

Set $J=M^{*}=M^{* * *} \subseteq R$; thus $F=\operatorname{End}_{R}(J)$ as well. Also, write $X=J J^{*}$, a nonzero ideal of $R$, and $W=J^{*} J$, a nonzero ideal of $F$. By Lemma 2.13(3), $J$ and $J^{*}=M^{* *}$ are $g$-divisible; in particular, $J \nsubseteq g T_{(g)}$ and $J^{*} \nsubseteq g T_{(g)}$. Thus, by Lemma 2.15(4), $\operatorname{GKdim}(R / X) \leq 1$ and $\operatorname{GKdim}(F / W) \leq 1$. By Assumption 8.2 and [RSS 2015, Proposition 8.7] we can choose a minimal sporadic ideal $X^{\prime}$ of $R$ such that $X^{\prime} \subseteq X$. Let $I=J^{*} X^{\prime} J$. Since $\operatorname{GKdim}\left(X^{\prime}\right) \leq 1$ and $R$ is $g$ divisible, GKdim $R / g R=2$ and so $X^{\prime} \nsubseteq g T_{(g)}$ also. Thus $I$ is an ideal of $F$ with $\operatorname{GKdim}(F / I) \leq 1$ by Lemma 2.15(4).

Now consider an arbitrary sporadic ideal $L$ of $F$, if such an ideal exists. Since $F$ is $g$-divisible, $L \nsubseteq g T_{(g)}$ and so, just as in the previous paragraph, $J L J^{*}$ is an ideal of $R$ satisfying $\operatorname{GKdim}_{R}\left(R / J L J^{*}\right) \leq 1$. Hence $J L J^{*} \supseteq X^{\prime} H$ for an ideal $H$ of $R$ with $\operatorname{dim}_{\mathbb{k}}(R / H)<\infty$. Now, $L \supseteq\left(J^{*} J\right) L\left(J^{*} J\right) \supseteq J^{*} X^{\prime} H J$, and [Krause and Lenagan 1985, Proposition 5.6] implies that $\operatorname{dim}_{\mathbb{k}}\left(J^{*} X^{\prime} J\right) /\left(J^{*} X^{\prime} H J\right)<\infty$. Thus $I$ is a minimal sporadic ideal of $F$.

In conclusion, $F$ and $V$ have a common ideal $K$ with $\operatorname{GKdim}(F / K) \leq 1$ (see Proposition 6.4). Thus $K I K$ is a minimal sporadic ideal for $F$ that lies in $V$ and so it is also a minimal sporadic ideal for $V$. 
Proposition 8.4. Suppose that $T$ satisfies Assumptions 2.1 and 8.2. Let $U \subseteq T$ be a g-divisible graded algebra with $Q_{\mathrm{gr}}(U)=Q_{\mathrm{gr}}(T)$. Then $U$ has a minimal sporadic ideal.

Proof. By Theorem 7.4(2), $U$ is contained in and equivalent to some $g$-divisible maximal $T$-order $V$, say with $a V b \subseteq U$ for some nonzero homogeneous $a, b \in U$. Set $U^{\prime}=U+U a V \subseteq V$, and $W=\widehat{U}^{\prime}$. Thus $a V \subseteq U^{\prime} \subseteq W$ and $U^{\prime} b \subseteq U$. By Lemma 2.13(1), $W b=\widehat{U^{\prime} b} \subseteq \widehat{U}=U$. Set $J=\ell-\operatorname{ann}_{W} V / W$, noticing that $J$ is a nonzero ideal of $W$ (since $a \in J$ ) and a right ideal of $V$. Also, as $W$ is $g$-divisible, it follows that $J$ is $g$-divisible. Thus, by Lemma 2.15(3), GKdim $W / J \leq 1$.

If $K$ is a minimal sporadic ideal in $V$ given by Lemma 8.3, we claim that $J K$ is a minimal sporadic ideal in $W$. To see this, let $L$ be any ideal of $W$ with GKdim $W / L \leq 1$. Then $I=V L J$ is an ideal of $V$. Since none of $V, L$, or $J$ is contained in $g T_{(g)}$, GKdim $V / I \leq 1$ by Lemma 2.15(4). Hence $I \supseteq K M$ for some ideal $M$ of $V$ with $\operatorname{dim}_{\mathbb{k}}(V / M)<\infty$ and so $L \supseteq J V L J \supseteq J K M$. This implies that $J K$ is a minimal sporadic ideal for $W$. Finally, a symmetric argument, using the fact that $W$ is $g$-divisible with a minimal sporadic ideal, proves that $U$ has such an ideal.

As in Section 7, results on $g$-divisible rings have close analogues for subalgebras of $T^{\circ}$.

Corollary 8.5. Suppose that $T$ satisfies Assumptions 2.1 and 8.2. Let $A$ be a subalgebra of $T^{\circ}$ with $Q(A)=Q\left(T^{\circ}\right)$. Then A has a unique minimal nonzero ideal $I$, and $\operatorname{dim}_{\mathbb{k}} A / I<\infty$. Further, A has DCC on ideals and finitely many primes.

Proof. Recall from Section 7 that there is a one-to-one correspondence between $g$-divisible ideals of $\Omega A$ and ideals of $A$. Since every nonzero $g$-divisible ideal of $\Omega A$ is sporadic, when combined with Proposition 8.4 this gives the existence of $I$ as described. Since $A / I$ is artinian it has finitely many prime ideals and DCC on ideals. Thus the same holds for $A$.

We now turn to a more general subalgebra $U$ of $T$, with the aim of controlling its sporadic ideals also. We achieve this by relating $U$ to its $g$-divisible hull $\widehat{U}$ and we begin with a straightforward lemma on subalgebras of TCRs. Recall that, for any subalgebra $U \subseteq T_{(g)}$, we write $\bar{U}=U+g T_{(g)} / T_{(g)}$.

Lemma 8.6. Let $B=B(E, M, \tau)$ for some smooth elliptic curve $E$, invertible sheaf $M$ of degree $d>0$ and $\tau$ of infinite order. Then for any $0 \neq x \in B_{k}$ we have $B_{n} x+x B_{n}=B_{n+k}$ for $n \gg 0$.

In particular, if $A$ is a graded subalgebra of $B$ such that $A \neq \mathbb{k}$, then $B$ is a noetherian ( $A, A)$-bimodule. 
Proof. By [Artin and Van den Bergh 1990, Theorem 1.3] and its left-right analogue, there exist effective divisors $\boldsymbol{x}$ and $\boldsymbol{x}^{\prime}$ such that

$$
x B_{\geq n_{0}}=\bigoplus_{n \geq n_{0}} H^{0}\left(E, \mu_{n+k}(-\boldsymbol{x})\right) \quad \text { and } \quad\left(B_{\geq n_{0}}\right) x=\bigoplus_{n \geq n_{0}} H^{0}\left(E, \mathcal{M}_{n+k}\left(-\tau^{-n} \boldsymbol{x}^{\prime}\right)\right)
$$

(With a little thought one can see that this holds with $n_{0}=0$ and $\boldsymbol{x}=\boldsymbol{x}^{\prime}$, but that is not relevant here.) Since $|\tau|=\infty$, we may choose $n_{0}$ so that $\boldsymbol{x} \cap \tau^{-n} \boldsymbol{x}^{\prime}=\varnothing$ for all $n \geq n_{0}$. For such $n$ there is an exact sequence

$$
0 \rightarrow \mathscr{O}_{E}\left(-x-\tau^{-n} x^{\prime}\right) \rightarrow \mathscr{O}_{E}(-x) \oplus \mathscr{O}_{E}\left(-\tau^{-n} x^{\prime}\right) \rightarrow \mathscr{O}_{E} \rightarrow 0 .
$$

Tensoring with $M_{n+k}$ and taking global sections gives a long exact sequence that reads, in part,

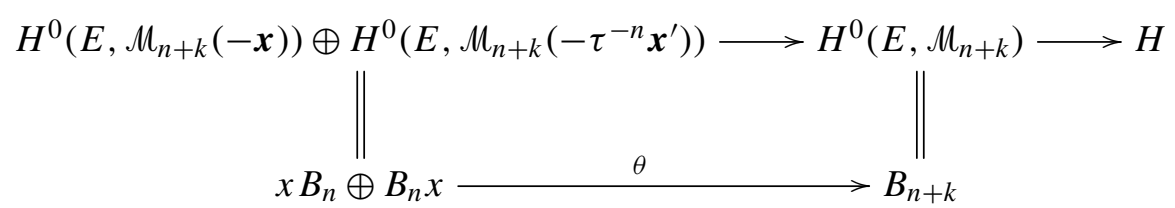

for $H=H^{1}\left(E, \mathcal{M}_{n+k}\left(-\boldsymbol{x}-\tau^{-n} \boldsymbol{x}^{\prime}\right)\right)$ and $\theta$ the natural map. Since

$$
\operatorname{deg}\left(M_{n+k}\left(-\boldsymbol{x}-\tau^{-n} \boldsymbol{x}\right)\right)>0 \text { for } n \gg 0,
$$

Riemann-Roch ensures that $H=0$ and hence that $\theta$ is surjective for such $n$.

This implies that $B$ is a noetherian $(\mathbb{k}\langle x\rangle, \mathbb{k}\langle x\rangle)$-bimodule, which certainly suffices to prove the final assertion of the lemma.

We now show that, under mild hypotheses, $\widehat{U}$ is equivalent to $U$. In this result the hypothesis that $\bar{U} \neq \mathbb{k}$ is annoying but necessary (see Example 10.8) but, as will be shown in Section 9, there are ways of circumventing it.

Proposition 8.7. Suppose that $T$ satisfies Assumptions 2.1 and 8.2. Let $U$ be a $\mathrm{cg}$ subalgebra of $T$ with $Q_{\mathrm{gr}}(U)=Q_{\mathrm{gr}}(T), g \in U$ and $\bar{U} \neq \mathbb{k}$.

(1) There exists $n \geq 0$ such that $U \cap T g^{m}=\widehat{U} \cap T g^{m}=g^{m} \widehat{U}$ for all $m \geq n$. Thus $U$ and $\widehat{U}$ are equivalent orders.

(2) If $U$ is right noetherian then $\widehat{U}$ is a finitely generated right $U$-module.

Proof. (1) Let $V=\widehat{U}$. Since $T$ is $g$-divisible, $V \subseteq T$. Working inside $Q_{\mathrm{gr}}(T)$, we get

$$
\left\{x \in T: x g^{k} \in U\right\}=g^{-k} U \cap T,
$$

and hence $V=\bigcup_{k \geq 0} g^{-k} U \cap T$. Now define $Q^{(k)}=\left(g^{-k} U \cap T+g T\right) / g T \subseteq \bar{T}$. Then, since $g \in U$,

$$
\bar{U}=Q^{(0)} \subseteq Q^{(1)} \subseteq \cdots \subseteq \bigcup_{k} Q^{(k)}=\bar{V}
$$


Each $Q^{(i)}$ is an $\bar{U}$-subbimodule of $\bar{T}$ and so, by Lemma 8.6, $Q^{(n)}=\bar{V}$ for some $n$.

We claim that $U \cap T g^{m}=V \cap T g^{m}$ for all $m \geq n$. If not, there exists $y=x g^{m} \in$ $V \cap T g^{m} \backslash U$ for some such $m$. Choose $x$ of minimal degree with this property. This ensures that $y \notin g^{m+1} T$, since otherwise one could write $y=g^{m+1} x^{\prime}$ with $\operatorname{deg}\left(x^{\prime}\right)=\operatorname{deg}(x)-1$. Since $\bar{x}=[x+g T] \in \bar{V}=Q^{(n)}$, we have $\bar{x}=\bar{w}$, where $w g^{n} \in U$. Thus $w g^{n}-x g^{n} \in V \cap T g^{n+1}$ and so $w-x=v g$, where $v g^{n+1} \in V \cap T g^{n+1}$. Since $\operatorname{deg} v<\operatorname{deg} x$, the minimality of $\operatorname{deg} x$ ensures that $v g^{n+1} \in U$. Then $x g^{n}=w g^{n}-$ $v g^{n+1} \in U$, and so $y=x g^{n}\left(g^{m-n}\right) \in U$, a contradiction. Thus $U \cap T g^{m}=V \cap T g^{m}$ as claimed. Finally, as $g V=V \cap g T$, an easy induction shows that $V \cap T g^{m}=g^{m} V$.

(2) This is immediate from part (1).

In the next result, we construct an ideal with a property that is slightly weaker than being a minimal sporadic ideal. However, it will have the same consequences.

Corollary 8.8. Suppose that $T$ satisfies Assumptions 2.1 and 8.2. Let $C$ be a $\mathrm{cg}$ subalgebra of $T$ with $Q_{\mathrm{gr}}(C)=Q_{\mathrm{gr}}(T)$. Assume that $g \in C$ and $\bar{C} \neq \mathbb{k}_{\text {. Then } C}$ has a sporadic ideal $K$ (possibly $K=C$ ) that is minimal among sporadic ideals $I$ for which $C / I$ is g-torsionfree.

Proof. Note that $\widehat{C}$ is noetherian by Proposition 2.9 and has a minimal sporadic ideal, say $J$, by Proposition 8.4. By Lemma $2.15(2), \widehat{J}$ is also a minimal sporadic ideal of $\widehat{C}$. Thus, replacing $J$ by $\widehat{J}$, we can assume that $\widehat{C} / J$ is $g$-torsionfree.

We will show that $K=J \cap C$ satisfies the conclusion of the corollary. So, let $I$ be a sporadic ideal of $C$ such that $C / I$ is $g$-torsionfree (if such an ideal exists). We first show that $J \cap C \subseteq I$. By Proposition 8.7, $H=g^{n} \widehat{C} \subseteq C$ for some $n \geq 1$

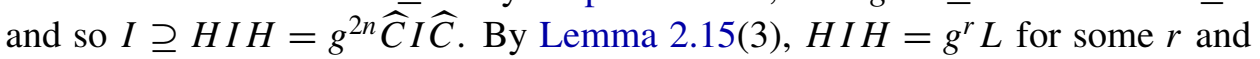
ideal $L$ of $\widehat{C}$ with $\operatorname{GKdim}(\widehat{C} / L) \leq 1$. As $J$ is sporadic, $\operatorname{dim}_{\mathbb{k}} J /(J \cap L)<\infty$ and so $L \cap J \supseteq J_{\geq s} \supseteq g^{s} J$ for some integer $s$. Combining these observations shows that $I \supseteq g^{t} J$ for some integer $t$. Pick $u$ minimal such that $I \supseteq g^{u}(J \cap C)$. If $u \neq 0$, then

$$
\frac{I+g^{u-1}(J \cap C)}{I}=\frac{I+g^{u-1}(J \cap C)}{I+g^{u}(J \cap C)}
$$

is $g$-torsion, and hence zero since $C / I$ is $g$-torsionfree by assumption. Hence $u=0$ and $I \supseteq J \cap C$.

It remains to show that $\mathrm{GKdim} C /(C \cap J) \leq 1$. Since $\widehat{C} / J$ is $g$-torsionfree, Lemma 2.14 implies that $M=\widehat{C} / J$ is a finitely generated $\mathbb{k}[g]$-module. Then the $C$-submodule $(C+J) / J \cong C /(J \cap C)$ is also. Therefore, by [Krause and Lenagan 1985, Corollary 5.4],

$$
\operatorname{GKdim}_{C}(C /(C \cap J))=\operatorname{GKdim}_{\mathbb{k}[g]}(C /(C \cap J)) \leq 1 .
$$

Thus $K=J \cap C$ satisfies the conclusions of the corollary. 
Lemma 8.9. The set of orders $\{C \subseteq T$ with $\bar{C} \neq \mathbb{k}$ and $g \in C\}$ satisfies $A C C$.

Proof. By Zorn's lemma, it suffices to prove that any such ring $C$ is finitely generated as an algebra; equivalently, that $C_{\geq 1}$ is finitely generated as a right ideal.

We first show that, for any $m \geq 1, C /\left(g^{m} T \cap C\right)$ is finitely generated as an algebra. The result holds for $m=1$ by [RSS 2014, Theorem 1.1(1)]. By induction, choose $a_{1}, \ldots, a_{k} \in C_{\geq 1}$ whose images generate $C /\left(g^{m-1} T \cap C\right)$ as an algebra. Set $X=\left(g^{m-1} T \cap C\right) /\left(g^{m} T \cap C\right)$. Then, up to shifts,

$$
X \cong \frac{T \cap g^{1-m} C}{g T \cap g^{1-m} C} \cong \frac{\left(T \cap g^{1-m} C\right)+g T}{g T} \subseteq T / g T
$$

as $\bar{C}$-bimodules. Thus, by Lemma $8.6, X$ is a finitely generated $\bar{C}$-bimodule, say by the images of $b_{1}, \ldots, b_{n} \in g^{m-1} T \cap C$. Then $\left\{a_{1}, \ldots, a_{k}, b_{1}, \ldots, b_{n}\right\}$ generate $C /\left(g^{m} T \cap C\right)$, completing the induction.

By Proposition 8.7, there exists $\ell \in \mathbb{N}$ such that $g^{m} \widehat{C}=C \cap g^{m} T \subseteq C$ for all $m \geq \ell$. By the above, choose $c_{1}, \ldots, c_{N} \in C_{\geq 1}$ whose images generate $C / g^{\ell+1} \widehat{C}$ as an algebra. Then for any $f \in C_{\geq 1}$, there exists $x \in \sum c_{i} C$ so that $f-x \in g^{\ell+1} \widehat{C}=$ $g\left(g^{\ell} \widehat{C}\right)$; thus $f \in g C+\sum c_{i} C$. Therefore, $C_{\geq 1}$ is generated as a right ideal by $g, c_{1}, \ldots, c_{N}$.

Proposition 8.10. Suppose that $T$ satisfies Assumptions 2.1 and 8.2. Let $U$ be a cg subalgebra of $T$ with $\bar{U} \neq \mathbb{k}$ and $D_{\mathrm{gr}}(U)=D_{\mathrm{gr}}(T)$. Then there exists a nonzero ideal of $C=U\langle g\rangle$ that is finitely generated as both a left and a right $U$-module.

Proof. By Lemma 8.9, there is a finitely generated cg subalgebra $W$ of $U$ with $C=U\langle g\rangle=W\langle g\rangle$. Note that $Q_{\mathrm{gr}}(C)=Q_{\mathrm{gr}}(T)$ as $g \in C$.

Fix $n \in \mathbb{N}$. Observe that $C W_{\geq n}=\sum_{m} W_{\geq n} g^{m}=W_{\geq n} C$ is an ideal of $C$. Moreover, $C / C W_{\geq n}$ is a homomorphic image of the polynomial ring $\left(W / W_{\geq n}\right)[g]$. Since $\operatorname{dim}_{\mathbb{k}}\left(W / W_{\geq n}\right)<\infty$, it follows that $C / C W_{\geq n}$ is a finitely generated $\mathbb{k}[g]$-module. In particular, by [Krause and Lenagan 1985, Corollary 5.4],

$$
\operatorname{GKdim}_{C}\left(C / C W_{\geq n}\right)=\operatorname{GKdim}_{\mathbb{k}[g]}\left(C / C W_{\geq n}\right) \leq 1 .
$$

Moreover, $K_{n}=\operatorname{tors}_{g}\left(C / C W_{\geq n}\right)$ is finite-dimensional.

Let $Z_{n}=C \cap \widehat{C W_{\geq n}}$; thus $Z_{n} / C W_{\geq n}=K_{n}$. Note that $C / Z_{n}$ is a finitely generated torsion-free, hence free, $\mathbb{k}[g]$-module. Therefore, if $d_{n}$ denotes the rank of that free module, then

$$
\begin{aligned}
d_{n} & =\operatorname{dim}_{\mathbb{k}}\left(C / Z_{n}\right)_{m} & & \text { for } m \gg 0 \\
& =\operatorname{dim}_{\mathbb{k}}\left(C / C W_{\geq n}\right)_{m} & & \text { for } m \gg 0 .
\end{aligned}
$$

Also, $C W_{\geq n} \supseteq C W_{\geq n+1}$, whence $Z_{n} \supseteq Z_{n+1}$ and $d_{n} \leq d_{n+1}$. 
Let $J$ be a minimal sporadic ideal of $\widehat{C}$ such that $\widehat{C} / J$ is $g$-torsionfree; thus, by Corollary 8.8 and its proof, $C \cap J$ is minimal among sporadic ideals of $C$ such that the factor is $g$-torsionfree. By construction, each $Z_{n}$ is either sporadic or equal to $C$; in either case $Z_{n} \supseteq C \cap J$. Now $C /(C \cap J) \hookrightarrow \widehat{C} / J$, which, by Lemma 2.14 , has an eventually constant Hilbert series; say $\operatorname{dim}_{\mathbb{k}}(\widehat{C} / J)_{m}=N$ for all $m \gg 0$. Hence $\operatorname{dim}\left(C / Z_{n}\right)_{m} \leq N$ for all such $m$ and, in particular, $d_{n} \leq N$. Since $d_{n} \leq d_{n+1}$, it follows that $d_{n}=d_{n+1}$ for all $n \gg 0$; say for all $n \geq n_{0}$. Thus, by the last display, $C W_{\geq n} \doteq C W_{\geq n_{0}}$ for all $n \geq n_{0}$.

Finally, if $W$ is generated as an algebra by elements of degree at most $e$, then $C W_{\geq n_{0}} W_{\geq 1} \supseteq C W_{\geq n_{0}+e}$. By the last paragraph, $\operatorname{dim}_{\mathbb{k}}\left(C W_{\geq n_{0}} / C W_{\geq n_{0}+e}\right)<\infty$, and so $\operatorname{dim}_{\mathbb{k}}\left(C W_{\geq n_{0}} / C W_{\geq n_{0}} W_{\geq 1}\right)<\infty$. Thus, by the graded Nakayama's lemma, $C W_{\geq n_{0}}=W_{\geq n_{0}} C=W_{\geq n_{0}} U\langle g\rangle$ is finitely generated as a right $W$-module, and hence as a right $U$-module.

Finally, we can reap the benefits of the last few results.

Theorem 8.11. Suppose that $T$ satisfies Assumptions 2.1 and 8.2. For some $n \geq 1$, let $U$ be a cg maximal $T^{(n)}$-order with $\bar{U} \neq \mathbb{k}$. Then $U$ is strongly noetherian; in particular, noetherian and finitely generated as an algebra. Moreover:

(1) If $n=1$, so $Q_{\mathrm{gr}}(U)=Q_{\mathrm{gr}}(T)$, then $U$ is $g$-divisible and $U=F \cap T$, where $F$ is a blowup of $T$ at a virtually effective divisor $\boldsymbol{x}=\boldsymbol{u}-\boldsymbol{v}+\tau^{-1}(\boldsymbol{v})$ of degree $<\mu$.

(2) If $Q_{\mathrm{gr}}(U) \neq Q_{\mathrm{gr}}(T)$, then there is a virtually effective divisor $\boldsymbol{x}$ of degree $<\mu$ and a blowup $F$ of $T$ at $\boldsymbol{x}$ so that $U=(F \cap T)^{(n)}$.

Proof. (1) Let $C=U\langle g\rangle$; thus $Q_{\mathrm{gr}}(U)=Q_{\mathrm{gr}}(\widehat{C})=Q_{\mathrm{gr}}(T)$. By Proposition 8.10, there exists an ideal $X$ of $C$ that is finitely generated as a right $U$-module. In particular, as $U$ is a right Ore domain and $X \subseteq Q_{\mathrm{gr}}(U)$, we can clear denominators from the left to find $q \in Q_{\mathrm{gr}}(U)$ such that $X \subseteq q U$. As $X$ is an ideal of $C$, we have $p C \subseteq X$ for any $0 \neq p \in X$ and hence $C \subseteq p^{-1} q U$. Thus $C$ and $U$ are equivalent orders. By Proposition 8.7 it follows that $U$ and $\widehat{C}$ are equivalent orders and hence $U=\widehat{C}$. Now apply Proposition 2.9 and Theorem 7.4.

(2) Keep $C$ and $X$ as above. In this case, as $Q_{\mathrm{gr}}(U)=\mathbb{k}(E)\left[g^{n}, g^{-n}, \tau^{n}\right]$, clearly $U$ and $C^{\prime}=U\left\langle g^{n}\right\rangle$ have the same graded quotient ring and, moreover, $C^{\prime}=C^{(n)}$. Therefore $X^{(n)}$ is an ideal of $C^{(n)}$ which, since it is a $U$-module summand of $X$, is also finitely generated as a right (and left) $U$-module. The argument used in (1) therefore implies that $U$ and $C^{(n)}$ are equivalent orders and hence that $U=C^{(n)}$. In particular, $C=\sum_{i=0}^{n-1} g^{i} C^{(n)}$ is a finitely generated right $U$-module.

Consider $\widehat{C}$. As $g \in C$, we have $Q_{\mathrm{gr}}(\widehat{C})=Q_{\mathrm{gr}}(T)$ and so, by Corollary 7.6(1), there exists a cg maximal $T$-order $V=\widehat{V} \subseteq T$ containing and equivalent to $\widehat{C}$. By 
Proposition 8.7, $V$ is equivalent to $C$. Further, $V=F \cap T$ where $F$ is a blowup of $T$ at some virtually effective divisor $\boldsymbol{x}$ on $E$ with $\operatorname{deg} \boldsymbol{x}<\mu$.

Now, $a V b \subseteq C$ for some $a, b \in C \backslash\{0\}$. By multiplying by further elements of $C$ we may suppose that $a, b \in C^{(n)}=U$ and hence that $a V^{(n)} b \subseteq U$. As $U$ is a maximal $T^{(n)}$-order, and certainly $V^{(n)} \subseteq T^{(n)}$, it follows that $U=V^{(n)}$.

One consequence of the theorem is that maximal $T^{(n)}$-orders have a number of pleasant properties, as we next illustrate. The undefined terms in the following corollary can be found in [Rogalski 2011, §2] and [Van den Bergh 1997].

Corollary 8.12. Suppose that $T$ satisfies Assumptions 2.1 and 8.2. For some $n \geq 1$, let $U$ be a cg maximal $T^{(n)}$-order with $\bar{U} \neq \mathbb{k}$. Then qgr- $U$ has cohomological dimension $\leq 2$, while $U$ has a balanced dualising complex and satisfies the ArtinZhang $\chi$ conditions.

Proof. By Theorem 8.11, $U=V^{(n)}$ for a $g$-divisible maximal $T$-order $V$. Hence $\bar{V} \doteq B(E, \mathcal{N}, \tau)$, by Theorem 6.7. Thus [Rogalski 2011, Lemma 2.2] and [Artin and Zhang 1994, Lemma 8.2(5)] imply that qgr- $\bar{V}$ has cohomological dimension one, and that $\bar{V}$ satisfies $\chi$. The fact that $V$ satisfies $\chi$ and that qgr- $V$ has cohomological dimension $\leq 2$ then follow from [ibid., Theorem 8.8]. By [Artin and Stafford 1995, Lemma 4.10(3)], $V$ is a noetherian $U$-module and so, by [Artin and Zhang 1994, Proposition 8.7(2)], these properties then descend to $U$. (With a little more work one can show that qgr- $V$ and qgr- $U$ have cohomological dimension exactly 2.) Finally, by [Van den Bergh 1997, Theorem 6.3], this implies the existence of a balanced dualising complex.

Let $U$ be a maximal order in $T$ with $\bar{U} \neq \mathbb{k}$. Theorem 8.11 also allows us to determine the simple objects in qgr- $U$, although we do not formalise their geometric structure.

Corollary 8.13. Suppose that $T$ satisfies Assumptions 2.1 and 8.2. Let $U$ be a cg maximal $T$-order with $\bar{U} \neq \mathbb{k}$. Then the simple objects in qgr- $U$ are in one-toone correspondence with the closed points of the elliptic curve E together with a (possibly empty) finite set.

Proof. A simple object in qgr- $U$ equals $\pi(M)$ for a cyclic critical right $U$-module $M$ with the property that every proper factor of $M$ is finite-dimensional. Suppose first that $M$ is $g$-torsion; thus $M g=0$ by Lemma 3.8. Hence, by Theorems 8.11 and 7.4, $\pi(M) \in$ qgr- $B$ for some TCR $B=B(E, \mathcal{N}, \tau)$. Thus, under the equivalence of categories qgr- $B \simeq \operatorname{coh}(E), \pi(M)$ corresponds to a closed point of $E$.

On the other hand, if $M$ is not annihilated by $g$, then Lemma 3.8 implies that $M$ is $g$-torsionfree. By comparing Hilbert series, it follows that $\operatorname{GKdim}(M / M g)=$ $\operatorname{GKdim}(M)-1$ and so, as $\operatorname{dim}_{\mathbb{k}} M / M g<\infty$ by construction, $\operatorname{GKdim}(M)=1$. In particular, $M^{\prime}=M\left[g^{-1}\right]_{0}$ is then a finite-dimensional simple $U^{\circ}$-module and hence 
is annihilated by the minimal nonzero ideal of $U^{\circ}$ (see Corollary 8.5). Pulling back to $U$, this says that $M$ is killed by the minimal sporadic ideal $K$ of $U$. Thus, by Lemma 3.8, $P=\mathrm{r}$-ann $(M)$ is one of the finitely many prime ideals $P$ minimal over $K$.

In order to complete the proof we need to show that $\pi(M)$ is uniquely determined by $P$. Note that, as $\operatorname{dim}_{\mathbb{k}}(M / M g)<\infty$, we have $\pi(M) \cong \pi(M g)=\pi(M[-1])$ in qgr- $U$, and so we do not need to worry about shifts. Next, as $\operatorname{GKdim}(M)=$ $\operatorname{GKdim}(U / P), M$ is a (Goldie) torsion-free $U / P$-module and hence is isomorphic to (a shift of) a uniform right ideal $J$ of $U / P$. However, given a second uniform right ideal $J^{\prime} \subseteq U / P$, then $J^{\prime}$ is isomorphic to (a shift of) a submodule $L \subseteq J$ (use the proof of [McConnell and Robson 2001, Corollary 3.3.3]). Once again, $\operatorname{dim}_{\mathbb{k}}(J / L)<\infty$ and so $\pi(J) \cong \pi\left(J^{\prime}\right)$, as required.

Corollary 8.14. Suppose that $T$ satisfies Assumptions 2.1 and 8.2. Let $U \subseteq T$ be a noetherian cg algebra with $D_{\mathrm{gr}}(U)=D_{\mathrm{gr}}(T)$ and $\bar{U} \neq \mathbb{k}$. Then $C=U\langle g\rangle$ and $\widehat{C}$ are both finitely generated right (and left) $U$-modules.

Proof. Again, let $X=C U_{\geq n_{0}}$ be the ideal of $C$ that is finitely generated as a right $U$-module given by Proposition 8.10. In this case, $X$ is a noetherian right $U$-module and hence so is $C \cong x C[n]$ for any $0 \neq x \in X_{n}$. The rest of the result follows from Proposition 8.7.

\section{Arbitrary orders}

The assumption $\bar{U} \neq k$ that appeared in most of the results from Section 8 is annoying but, as Example 10.8 shows, necessary. Fortunately one can bypass the problem, although at the cost of passing to a Veronese ring. In this section we explain the trick and apply it to describe arbitrary cg orders in $T$.

Up to now graded homomorphisms of algebras have been degree-preserving, but this will not be the case for the next few results, and so we make the following definition. A homomorphism $A \rightarrow B$ between $\mathbb{N}$-graded algebras is called graded of degree $t$ if $\phi\left(A_{n}\right) \subseteq B_{n t}$ for all $n$. The map $\phi$ is called semigraded if it is graded of degree $t$ for some $t$.

Proposition 9.1. Suppose that $T$ satisfies Assumption 2.1 and that $U$ is a cg noetherian subalgebra of $T$ with $U \nsubseteq \mathbb{k}[g]$. Then there exist $N, M \in \mathbb{N}$ and an injective graded homomorphism $\phi: U^{(N)} \rightarrow T$ of degree $M$ such that $U^{\prime}=\phi\left(U^{(N)}\right) \nsubseteq \mathbb{k}+g T$. In addition, $D_{\mathrm{gr}}(U)=D_{\mathrm{gr}}\left(U^{\prime}\right) \subseteq D_{\mathrm{gr}}(T)$.

Proof. For $n \geq 0$, define $f: \mathbb{N} \rightarrow \mathbb{N} \cup\{-\infty\}$ by

$$
f(n)=\min \left\{i: U_{n} \subseteq g^{n-i} T\right\}, \quad \text { with } f(n)=-\infty \text { if } U_{n}=0 .
$$


Trivially, $f(n) \in\{0,1, \ldots, n\} \cup\{-\infty\}$ for all $n \geq 0$, and $f(n)=0$ if and only if $U_{n}=\mathbb{k} g^{n}$.

We first claim that $f(n)+f(m) \leq f(n+m)$ for all $m, n \geq 0$. As $A$ is a domain, this is clear if one of the terms equals $-\infty$, and so we may assume that $f(r) \geq 0$ for $r=n, m, n+m$. Write $U_{r}=X_{r} g^{r-f(r)}$ for such $r$; thus $X_{r} \subseteq T$ but $X_{r} \nsubseteq g g T$. Since $g T$ is a completely prime ideal, $X_{n} X_{m} \subseteq T$ but $X_{n} X_{m} \nsubseteq g T$. In other words, $U_{n} U_{m} \nsubseteq Y Y=g^{(n-f(n)+m-f(m)+1)} T$. Since $U_{n} U_{m} \subseteq U_{n+m}$ it follows that $U_{n+m} \nsubseteq Y$ and hence that $f(n+m) \geq f(n)+f(m)$, as claimed.

A noetherian cg algebra is finitely generated by the graded Nakayama's lemma, so suppose that $U$ is generated in degrees $\leq r$. Then $U_{n}=\sum_{i=1}^{r} U_{i} U_{n-i}$ for all $n>r$. Arguing as in the previous paragraph shows that

$$
f(n)=\max \{f(n-i)+f(i): 1 \leq i \leq r\} \text { for } n>r,
$$

with the obvious conventions if any of these numbers equals $-\infty$.

We claim that there exists $N$ with $f(N)>0$ such that $f(n N)=n f(N)$ for all $n \geq 1$. This follows by exactly the same proof as in [Artin and Stafford 1995, Lemma 2.7]. Namely, choose $1 \leq N \leq r$ such that $\lambda=f(N) / N$ is as large as possible; by induction using (9.2) it follows that $f(n) \leq \lambda n$ for all $n \geq 0$, and this forces $f(n N)=n f(N)$ for all $n \geq 0$, as claimed.

Let $M=f(N)$ and note that $M>0$ since $U \nsubseteq \mathbb{k}[g]$. Thus, for each $n \geq 0$ we have $U_{n N} \subseteq g^{n N-n M} T$ but $U_{n N} \nsubseteq g^{n N-n M+1} T$. Therefore the function $U_{n N} \rightarrow T_{n M}$ given by $x \mapsto x g^{n(M-N)}$ is well-defined, and it defines an injective vector space homomorphism $\theta: U^{(N)} \rightarrow T$ with $\theta\left(U^{(N)}\right) \nsubseteq \mathbb{k}+g T$. It is routine to see that $\theta$ is an algebra homomorphism which is graded of degree $M$. The final claim of the proposition is clear because $D_{\mathrm{gr}}(U)=D_{\mathrm{gr}}\left(U^{(N)}\right)=D_{\mathrm{gr}}\left(U^{\prime}\right)$.

Corollary 9.3. (1) Suppose $T$ satisfies Assumption 2.1 and that $U$ is a noetherian subring of $T$ generated in a single degree $N$, with $U \neq \mathbb{k}\left[g^{N}\right]$. Then up to a semigraded isomorphism we may assume that $U \nsubseteq \mathbb{k}+g T$.

(2) Suppose also that $T$ satisfies Assumption 8.2. If $U$ is a noetherian maximal $T^{(N)}$-order generated in degree $N$ then, again up to a semigraded isomorphism, $U \cong V^{(M)}$, where $(V, F)$ is a maximal order pair and $M \leq N$.

Proof. In this proof, Veronese rings are unregraded; that is, they are given the grading induced from $T$.

(1) Pick $M \in \mathbb{N}$ minimal such that $U_{N} \subseteq g^{N-M} T$. Necessarily, $M \leq N$. Then, either directly or by Proposition 9.1, there is a semigraded monomorphism

$$
\phi: U=U^{(N)} \rightarrow T \text { given by } u \mapsto g^{M-N} u \text { for } u \in U_{N} .
$$

Hence $U \cong \phi(U)$, and $\phi(U) \nsubseteq \mathbb{k}+g T$ by the choice of $M$. 
(2) As $U$ is an order in $T^{(N)}$, certainly $\phi(U)$ is an order in $T^{(M)}$. So, suppose that $\phi(U) \subseteq W \subseteq T^{(M)}$ for some equivalent order $W$; say with $a W b \subseteq \phi(U)$, for $a, b \in \phi(U)$. Since $M \leq N$, the map $\phi^{-1}$ extends to give a well-defined semigraded homomorphism $\psi: T^{(M)} \rightarrow T^{(N)}$ defined by $\gamma \mapsto g^{N-M} \gamma$ for all $\gamma \in T_{M}$. Therefore, $\psi(a) U \psi(b) \subseteq U \subseteq \psi(W) \subseteq T^{(N)}$ and hence $U=\psi(W)$. Thus, $\phi(U)=W$ is a maximal order in $T^{(M)}$ with $\phi(U) \notin \mathbb{k}+g T$. Now apply Theorem 8.11(2).

One question we have been unable to answer is the following.

Question 9.4. Suppose that $U \subseteq T$ is a cg maximal $T$-order or, indeed, a maximal order. Then is each Veronese ring $U^{(n)}$ also a maximal $T^{(n)}$-order? The question is open even when $U$ is noetherian.

If this question has a positive answer, one can mimic the proof of Corollary 9.3 for any noetherian maximal order $U$ to get a precise description of some Veronese ring $U^{(N)}$. However, the best we can do at the moment is to use the much less precise result given by the next corollary, which also describes arbitrary noetherian cg subalgebras of $T$.

Corollary 9.5. Suppose that $T$ satisfies Assumptions 2.1 and 8.2. Let $U \subseteq T$ be a noetherian algebra with $D_{\mathrm{gr}}(U)=D_{\mathrm{gr}}(T)$. Then, up to taking Veronese subrings, $U$ is an iterated subidealiser inside a virtual blowup of $T$. More precisely, the following hold.

(1) There is a semigraded isomorphism of Veronese rings $U^{(N)} \cong U^{\prime}$, where $U^{\prime} \subseteq T$ is a noetherian algebra such that $D_{\mathrm{gr}}\left(U^{\prime}\right)=D_{\mathrm{gr}}(T)$ and $U^{\prime} \nsubseteq \mathbb{k}+g T$.

(2) If $C=U^{\prime}\langle g\rangle$ and $Z=\widehat{C}$, then $Z$ is a finitely generated (left and right) $U^{\prime}$ module and $Z$ is a noetherian algebra with $Q_{\mathrm{gr}}(Z)=Q_{\mathrm{gr}}(T)$. The g-divisible algebra $Z$ is described by Corollary 7.6.

Proof. By [Artin and Zhang 1994, Proposition 5.10], the Veronese ring $U^{(N)}$ is noetherian and so part (1) follows from Proposition 9.1. Part (2) then follows from Corollary 8.14 (and Corollary 7.6).

\section{Examples}

We end the paper with several examples that illustrate some of the subtleties involved here. For simplicity, these examples will all be constructed from $T=S^{(3)}$ for the standard Sklyanin algebra $S$ of Examples 2.2(1); thus $\mu=\operatorname{deg} M=9$.

We first construct a $g$-divisible, maximal $T$-order $U$ that is not a maximal order in $Q_{\mathrm{gr}}(U)$, as promised in Section 6. This shows, in particular, that the concept of maximal order pairs is indeed necessary in that section. In order to construct the example, we need the following notation. 
Notation 10.1. Fix $0 \neq x \in S_{1}$ and let $c=p+q+r$ be the hyperplane section of $E$ where $x$ vanishes. We can and will assume that no two of $p, q, r$ lie on the same $\sigma$-orbit on $E$, where $S / g S \cong B(E, \mathscr{L}, \sigma)$. Set $R=T(\boldsymbol{c})$. By [Rogalski 2011, Example 11.3], $R$ has a sporadic ideal $I=x S_{2} R$. Write $N=x T_{1} x^{-1} R$ and $M=x S_{5} R+R$. Finally, set $\boldsymbol{d}=\sigma^{-2}(\boldsymbol{c})=\sigma^{-2}(p)+\sigma^{-2}(q)+\sigma^{-2}(r)$ and hence $\boldsymbol{d}^{\tau}=\sigma^{-5}(\boldsymbol{c})$.

As we will see, $U=\operatorname{End}_{R}(M)$ will (essentially) be the required maximal $T$-order with equivalent maximal order being $F=\operatorname{End}_{R}(N)$. The proof will require some detailed computations, which form the content of the next lemma. We note that for subspaces of homogeneous pieces of $S$ we use the grading on $S$, but for subspaces that live naturally in $T$ we use the $T$-grading. For example, we write $T_{1} S_{2}=S_{5}$.

Lemma 10.2. Keep the data from Notation 10.1.

(1) $N I=x S_{5} R \subseteq M$ and $M_{\geq 1} \subseteq N$. Hence $N^{* *}=M^{* *}=(\widehat{M})^{* *}=\widehat{M^{* *}}$.

(2) $U^{\prime}=\operatorname{End}_{R}(\widehat{M}) \subseteq T$, but

(3) $F=\operatorname{End}_{R}\left(M^{* *}\right)=\operatorname{End}_{R}(N I)=x T\left(\boldsymbol{d}^{\tau}\right) x^{-1}$. Moreover, $F \nsubseteq T$.

Proof. (1) Clearly

$$
N I=x T_{1} S_{2} R=x S_{5} R \subseteq M=x S_{5} R+R .
$$

By [Rogalski 2011, Example 11.3], $R_{1}=x S_{2}+\mathbb{k} g$ and so $R_{1} x \subseteq x T$. Equivalently, $R_{1} \subseteq x T_{1} x^{-1} \subseteq N$. As $R=T(c)$ is generated in degree one by Proposition 4.10(2), $R \subseteq x T x^{-1}$. In particular, $M_{\geq 1}=x S_{5} R+R_{\geq 1} \subseteq N$. As $I$ is a sporadic ideal, it follows from Proposition 4.10 and Lemma 4.11(1) that $N^{* *}=(N I)^{* *}$ and hence that $M^{* *}=N^{* *}$.

Now consider $\widehat{M}$. Since $1 \in M$, certainly $M T=T$ and so $M^{* *}=(\widehat{M})^{* *}=\widehat{M^{* *}}$ by Lemma $2.13(3)$.

(2) Since $M T=T$ we have $\widehat{M} T=T$, from which the result follows.

(3) We will first prove that $\operatorname{End}_{R}(N)=x T\left(\boldsymbol{d}^{\tau}\right) x^{-1}$. As in (1), $R_{1}=x S_{2}+\mathbb{k} g$. Equivalently, $\left(x^{-1} R x\right)_{1}=S_{2} x+\mathbb{k} g$ is a 7-dimensional subspace of $T_{1}$ that vanishes at the points $\sigma^{-2}(p), \sigma^{-2}(q)$ and $\sigma^{-2}(r)$. Now, $T(\boldsymbol{d})_{1}$ is also 7-dimensional by [Rogalski 2011, Theorem 1.1(1)]. Consequently, $\left(x^{-1} R x\right)_{1}=T(\boldsymbol{d})_{1}$ and so $x^{-1} R x=T(d)$, since both algebras are generated in degree 1 by Proposition 4.10(2). Therefore,

$$
x^{-1} N x=T_{1}\left(x^{-1} R x\right)=T_{1} T(\boldsymbol{d})=T\left(\boldsymbol{d}^{\tau}\right) T_{1},
$$

where the final equality follows from [RSS 2015, Corollary 4.14]. Thus

$$
x T\left(\boldsymbol{d}^{\tau}\right) T_{1} x^{-1}=N
$$


and so $\operatorname{End}_{R}(N) \supseteq G=x T\left(\boldsymbol{d}^{\tau}\right) x^{-1}$. Since $N=G x T_{1} x^{-1}$, Lemma 6.3 implies that $\operatorname{End}_{R}(N)$ is a finitely generated left $G$-module. But $G$ is a maximal order by [Rogalski 2011, Theorem 1.1(2)], and so $\operatorname{End}_{R}(N)=G$. Thus, by part (1) and Lemma 6.2, $\operatorname{End}_{R}(N)=\operatorname{End}_{R}\left(N^{* *}\right)=\operatorname{End}\left(M^{* *}\right) . \operatorname{Moreover}_{\operatorname{End}_{R}}(N) \subseteq$ $\operatorname{End}_{R}(N I)$ and we again have equality by Lemma 6.3 .

It remains to prove that $x T\left(\boldsymbol{d}^{\tau}\right) x^{-1} \nsubseteq T$. This will follow if we show that $\bar{x} \bar{X} \bar{x}^{-1} \nsubseteq \bar{T}$, where $X=T\left(\boldsymbol{d}^{\tau}\right)$ and $\bar{X}=\left(X+g T_{(g)}\right) / T_{(g)}$. So, assume that $\bar{x} \bar{X} \bar{x}^{-1} \subseteq \bar{T}$. Then $\bar{x} \bar{X}_{1} \subseteq \bar{T}_{1} \bar{x}=\bar{S}_{3} \bar{x}$. However, inside $\bar{S}_{4}$,

$$
\bar{x} \bar{X}_{1} \subseteq H^{0}\left(E, \mathscr{L}_{4}\left(-p-q-r-\sigma^{-6}(p)-\sigma^{-6}(q)-\sigma^{-6}(r)\right)\right),
$$

and, since both are 6-dimensional, they are equal. On the other hand,

$$
\bar{S}_{3} \bar{x}=H^{0}\left(E, \mathscr{L}_{4}\left(-\sigma^{-3}(p)-\sigma^{-3}(q)-\sigma^{-3}(r)\right)\right) .
$$

Inside $\bar{S}_{4}$, vanishing conditions at $\leq 12$ distinct points give independent conditions. So there exists $z$ that vanishes at the first 6 points $p, \ldots, \sigma^{-6}(r)$ but not at the points $\sigma^{-3}(p), \sigma^{-3}(q), \sigma^{-3}(r)$. This implies that $\bar{x} \bar{X}_{1} \nsubseteq \bar{T}_{1} \bar{x}$, and completes the proof of the lemma.

We are now able to give the desired example.

Proposition 10.3. There exists a maximal order pair $(V, F)$ with $V \neq F$. In particular, $V$ is a maximal $T$-order that is not a maximal order.

In more detail, and using the data from Notation 10.1, $F=\operatorname{End}_{R}\left((\widehat{M})^{* *}\right)=$ $x T\left(\boldsymbol{d}^{\tau}\right) x^{-1}$ is a blowup of $T$ at $\boldsymbol{x}=\boldsymbol{c}-\tau^{-1}(\boldsymbol{c})+\tau^{-2}(\boldsymbol{c})$. The algebra $F$ is also Auslander-Gorenstein and CM.

Proof. As $1 \in M$, Theorem 6.7 and Lemma 10.2 imply that $F=\operatorname{End}_{R}\left((\widehat{M})^{* *}\right)=$ $x T\left(\boldsymbol{d}^{\tau}\right) x^{-1}$ is a maximal order with $F \nsubseteq T$. By Theorem 6.7, again, $V=T \cap F$ is a $g$-divisible maximal $T$-order, but $V$ is not a maximal order as $V \neq F$. That $F$ is Auslander-Gorenstein and CM follows from Proposition 4.10.

Theorem 6.7 also implies that $F$ is a blowup of $T$ at some virtual divisor $\boldsymbol{y}$, so it remains to check that $\boldsymbol{y}=\boldsymbol{x}$. By Lemma 10.2, $F=\operatorname{End}_{R}(N I)=\operatorname{End}_{R}\left(x S_{5} R\right)$ and hence $\bar{F} \subseteq \operatorname{End}_{\bar{R}}\left(\overline{x S_{5}} \bar{R}\right)$. Now, for any $n \geq 2$, one has

$$
\bar{R}_{n-2}=H^{0}\left(E, M\left(-c-c^{\tau}-\cdots-c^{\tau^{n-3}}\right)\right),
$$

and so

$$
\left(\overline{x S_{5}} \bar{R}\right)_{n}=H^{0}\left(E, M\left(-c-c^{\tau^{2}}-c^{\tau^{3}}-\cdots-c^{\tau^{n-1}}\right)=H^{0}\left(E, \mathcal{O}\left(\boldsymbol{c}^{\tau}\right) M(-c)_{n}\right) .\right.
$$

Hence

$$
\bar{F} \subseteq \operatorname{End}_{\bar{R}}\left(\overline{x S_{5} R}\right)=\operatorname{End}_{\bar{R}}\left(\bigoplus_{n \geq 2} H^{0}\left(E, \mathcal{O}\left(c^{\tau}\right) \mu(-c)_{n}\right)\right)
$$


Therefore, by Lemma 6.14(1), $\bar{F} \doteq B(E, M(-x), \tau)$. By [Rogalski 2011, Theorem 1.1(2)] and Riemann-Roch, $\operatorname{dim} \bar{F}_{n}=6 n=\operatorname{dim} B(E, M(-x), \tau)$, for $n \geq 1$, and hence $\bar{F}=B(E, M(-\boldsymbol{x}), \tau)$, as required.

When $\boldsymbol{y}$ is effective, the blowup $T(\boldsymbol{y})$ is both Auslander-Gorenstein and CM (see Proposition 4.10), as is the blowup of $T$ at $\boldsymbol{x}$ from Proposition 10.3. Despite this example, neither the Auslander-Gorenstein nor the CM condition is automatic for a blowup of $T$ at virtually effective divisors.

Example 10.4. Let $\boldsymbol{x}=p-\tau(p)+\tau^{2}(p)$ for a closed point $p \in E$ and let $U$ be a blowup of $T$ at $\boldsymbol{x}$. Then $U$ is a maximal order contained in $T$ that is neither Auslander-Gorenstein nor AS Gorenstein nor CM.

Proof. By Definition 6.9 and Corollary 6.6(2), $U=\operatorname{End}_{T(q)}(M)$, where $M=M^{* *}$ satisfies $M T=T$ and $q$ is a closed point that is $\tau$-equivalent to $\boldsymbol{x}$ and hence to $p$. By [RSS 2015, Example 9.5], $T(q)$ has no sporadic ideals and so, by Corollary 6.6(3), $U$ is a $g$-divisible maximal order contained in $T$.

Now consider $\bar{U}=U / g U$. By Theorem $6.7, \bar{U} \doteq B=B(E, M(-x), \tau)$. We emphasise that we always identify $M(-\boldsymbol{x})$ and $M$ with the appropriate subsheaves of the field $\mathbb{k}(E)$ and $B$ with the corresponding subring of the Ore extension $T_{(g)} / g T_{(g)} \cong \mathbb{k}(E)\left[z, z^{-1} ; \tau\right]$. We first want to show that $\bar{U} \neq B$. Since

$$
\operatorname{deg}(M(-x))=\operatorname{deg} M-\operatorname{deg} \boldsymbol{x}=8,
$$

[Hartshorne 1977, Corollary IV.3.2] implies that $M(-\boldsymbol{x})$ is very ample and generated by its sections $B_{1}=H^{0}(E, M(-\boldsymbol{x}))$. On the other hand, the inclusion $U \subseteq T$ forces $\bar{U} \subseteq \bar{T}=B(E, \mu, \tau)$ and again $\bar{T}_{1}$ generates $\mu$. Therefore, if $\bar{U}=B$ or even if $\bar{U}_{1}=B_{1}$ then $\mathcal{M}(-\boldsymbol{x}) \subseteq \mathcal{M}$. Since $\boldsymbol{x}$ is not effective, this is impossible and so $\bar{U} \neq B$, as claimed.

We now turn to the homological questions. By [Levasseur 1992, Theorem 5.10], $U$ is Auslander-Gorenstein, AS Gorenstein or CM if and only if the same holds for $\bar{U}$. Thus we can concentrate on $\bar{U}$. Since $B / \bar{U}$ is a nonzero, finite-dimensional vector space, and $B$ is a domain, certainly $\operatorname{Ext}_{\bar{U}}^{1}(\mathbb{k}, \bar{U}) \neq 0$ (on either side). Since $\operatorname{GKdim} \bar{U}=\mathrm{GKdim} B=2$ this certainly implies that $\bar{U}$ is not CM. Moreover, if we can prove that $\operatorname{Ext}_{\bar{U}}^{2}(\mathbb{k}, \bar{U}) \neq 0$ on either side, then $\bar{U}$ will be neither AS Gorenstein nor Auslander-Gorenstein.

By [Levasseur 1992, Proposition 6.5], $\operatorname{Ext}_{B}^{i}(\mathbb{k}, B)=\delta_{i, 2} \mathbb{k}$, up to a shift in degree. Therefore [Rotman 2009, Corollary 10.65], with $A=\mathbb{k}, B=S$ and $R=C=\bar{U}$, gives

$$
\operatorname{Ext}_{\bar{U}}^{2}(\mathbb{k}, \bar{U})=\operatorname{Ext}_{\bar{U}}^{2}\left(B \otimes_{B} \mathbb{k}, \bar{U}\right)=\operatorname{Ext}_{B}^{2}(\mathbb{k}, J) \text { for } J=\operatorname{Hom}_{\bar{U}}(B, \bar{U}) .
$$

Since $\bar{U} \doteq B$, clearly $L=B / J$ is also a nonzero finite-dimensional $\mathbb{k}$-vector space. We claim that the same is true of $\operatorname{Ext}_{B}^{2}(\mathbb{k}, J)$. As $\operatorname{Ext}_{B}^{1}(\mathbb{k}, B)=0$, we have an exact 
sequence

$$
0 \longrightarrow \operatorname{Ext}_{B}^{1}(\mathbb{k}, L) \longrightarrow \operatorname{Ext}_{B}^{2}(\mathbb{k}, J) \longrightarrow \operatorname{Ext}_{B}^{2}(\mathbb{k}, B) \longrightarrow \cdots .
$$

Since $\operatorname{dim}_{\mathbb{k}} \operatorname{Ext}_{B}^{1}(\mathbb{k}, L)<\infty$, the claim will follow once we show that $\operatorname{Ext}_{B}^{1}(\mathbb{k}, L) \neq 0$.

As in [Artin and Zhang 1994, (7.1.2)], let $I(L)$ denote the largest essential extension of $L$ by locally finite-dimensional modules. If $\operatorname{soc}(L)$ denotes the socle of $L$, then $L$ and $\operatorname{soc}(L)$ have the same injective hulls and hence the same torsion-injective hulls $I(L)=I(\operatorname{soc}(L))$. By [Rogalski 2011, Lemma 2.2(2)], $B$ satisfies $\chi$ in the sense of [Artin and Zhang 1994, Definition 3.2] and so, by [ibid., Proposition 7.7], $I(L)$ is a direct sum of copies of shifts of the vector space dual $B^{*}$. Since this is strictly larger than $L, \operatorname{Ext}_{B}^{1}(\mathbb{k}, L) \neq 0$ and the claim follows.

In conclusion, by (10.6) we know that $0<\operatorname{dim}_{\mathbb{k}} \operatorname{Ext}_{B}^{2}(\mathbb{k}, J)<\infty$ and hence by (10.5) it follows that (up to a shift) $\mathbb{k} \hookrightarrow \operatorname{Ext}_{\bar{U}}^{2}(\mathbb{k}, \bar{U})$ as left $\bar{U}$-modules. As noted earlier, this shows that both Gorenstein conditions fail.

Remark 10.7. (1) By expanding upon the above proof one can in fact show that $U$ from Example 10.4 will have infinite injective dimension.

(2) Explicit computation shows that $U$ is not uniquely determined by $\boldsymbol{x}$ as a subalgebra of $T$, although the factor $\bar{U}$ is determined in large degree. We do not know whether $U$ is unique up to isomorphism.

Let $U$ be a noetherian subring of $T$ with $Q_{\mathrm{gr}}(U)=Q_{\mathrm{gr}}(T)$. In Proposition 8.7, we had to assume that $U \nsubseteq \mathbb{k}+g T$ in order to find a $g$-divisible, equivalent order and this meant that the same assumption was needed for the rest of Section 8. In our next example we show that the conclusions of Proposition 8.7 can fail without this assumption, as does Theorem 8.11. Thus Proposition 9.1 is necessary for Section 9 .

In order to define the ring, pick algebra generators of $T$ in degree 1 ; say $T=$ $\mathbb{k}\left\langle a_{1}, \ldots, a_{r}\right\rangle$, set $T^{g}=\mathbb{k}\left\langle g a_{1}, \ldots, g a_{r}\right\rangle$ and write $U=T^{g}\langle g\rangle \subset T$ for the subring of $T$ generated by $T^{g}$ and $g$.

Example 10.8. Keep $T^{g}$ and $U=T^{g}\langle g\rangle$ as above. Then:

(1) There is a semigraded isomorphism $T^{g} \cong T$. Thus $U$ is noetherian and there is a semigraded isomorphism $T[x] /\left(x^{2}-g\right) \cong U$ mapping $x$ to $g$. Moreover, $U^{(2)}=T^{g}$ and so $U^{\circ}=\left(T^{g}\right)^{\circ} \cong T^{\circ}$.

(2) $U \subseteq \mathbb{k}+g T$ and so $\bar{U}=\mathbb{k}$.

(3) $g U$ is a prime ideal of $U$ such that there is a semigraded isomorphism $U / g U \cong$ $B=T / g T$.

(4) $\widehat{U}=T$ but $T$ is not finitely generated as a right (or left) $U$-module.

(5) $U$ is a maximal order with $Q_{\mathrm{gr}}(U)=Q_{\mathrm{gr}}(T)$.

Proof. (1-2) These are routine computations. 
(3) Under the identification $U=T[x] /\left(x^{2}-g\right)$, clearly $U / x U=T / g T$.

(4) For any $\theta \in T_{n}$ one has $g^{n} \theta \in T^{g} \subseteq U$ and hence $\widehat{U}=\widehat{T^{g}}=T$. If $T$ were finitely generated as a (right) $U$-module then the factor $B=T / g T$ would be finitely generated as a module over the image $(U+g T) / g T=\bar{U}$ of $U$ in $B$. This contradicts (2).

(5) Write $U=T[x] /\left(x^{2}-g\right)$; thus $x \in U_{1}$ but the grading of $T$ is shifted. If $U$ is not a maximal order then there exists a cg ring $U \subsetneq V \subset Q(U)$ such that either $a V \subseteq U$ or $V a \subseteq U$ for some $0 \neq a \in U$. By symmetry we may assume the former, in which case $I V=I$ for the nonzero ideal $I=U a V$ of $U$. Thus $I^{(2)} V^{(2)}=I^{(2)}$, and $I^{(2)} \neq 0$ since $U$ is a domain. Since $U^{(2)}=T$ is a maximal order by Proposition 4.10(4), it follows that $V^{(2)}=U^{(2)}=T$. Let $f \in V \backslash U$ be homogeneous. Then $f$ appears in odd degree and so $f x \in V^{(2)}=U^{(2)}=T$ and $f=t x^{-1}$ for some $t \in T$. However, $T=V^{(2)} \ni f^{2}=\left(t x^{-1}\right)^{2}=t^{2} g^{-1}$. Hence $t^{2} \in g T$ which, since $T / g T$ is a domain, forces $t=g t_{1} \in g T$. But this implies that $f=t x^{-1}=x t_{1} \in U$, a contradiction. Thus $U$ is indeed a maximal order. Moreover, as $g \in U$, clearly each $a_{i}$ lies in $Q_{\mathrm{gr}}(U)$ and hence $Q_{\mathrm{gr}}(T)=Q_{\mathrm{gr}}(U)$.

In this paper we have only been concerned with two-sided noetherian rings, since we believe that this is the appropriate context for noncommutative geometry. For one-sided noetherian rings there are further examples that can appear, as is illustrated by the following example.

Example 10.9. Let $J$ be a right ideal of $T$ such that $g \in J$ and $\operatorname{GKdim}(T / J)=1$. Then the idealiser $A=\llbracket(J)$ is right but not left noetherian.

Proof. Let $\bar{J}=J / g J$. Since $B=T / g T$ is just infinite [Rogalski 2011, Lemma 3.2], $\operatorname{dim}_{\mathbb{k}} T / T J<\infty$. Since $T J=\sum_{i=1}^{m} t_{i} J$ for some $t_{j}$, it follows that $T J$ and hence $T$ are finitely generated right $A$-modules. Thus, by the proof of [Stafford and Zhang 1994, Theorem 3.2], $A$ is right noetherian. On the other hand, $B$ is not a finitely generated left $A / g T$-module, and so $g T$ is an ideal of $A$ that cannot be finitely generated as a left $A$-module.

\section{Acknowledgements}

Part of this material is based upon work supported by the National Science Foundation under Grant No. 0932078 000, while the authors were in residence at the Mathematical Science Research Institute (MSRI) in Berkeley, California, during the spring semester of 2013. During this trip, Sierra was also partially supported by the Edinburgh Research Partnership in Engineering and Mathematics, and Stafford was partially supported by the Clay Mathematics Institute and Simons Foundation. The authors gratefully acknowledge the support of all these organisations. 


\section{Index of notation}

$\alpha$-pure

Allowable divisor layering $\boldsymbol{d}^{\bullet}$

Blowup at an arbitrary divisor

$\mathrm{CM}$ and Gorenstein conditions

$\boldsymbol{d}^{\tau}=\tau^{-1}(\boldsymbol{d})$ for a divisor $\boldsymbol{d}$

$\boldsymbol{d}_{n}=\boldsymbol{d}+\boldsymbol{d}^{\tau}+\cdots+\boldsymbol{d}^{\tau^{n-1}}$ for a divisor $\boldsymbol{d}$

2080

$D_{\mathrm{gr}}(A)$, function skewfield

$\mathscr{F}_{n}=\mathscr{F} \otimes \mathscr{F}^{\tau} \otimes \cdots \otimes \mathscr{F}^{\tau^{n-1}}$ for a sheaf $\mathscr{F}$

2063

2061

2064

2082

2063

2063

2069

2064

2077

2062

2076

2090

2088

2101

Left allowable divisor layering $\boldsymbol{d}^{\bullet}$

$\mu=\operatorname{deg} \mu$

Maximal order pair $(V, F)$

Maximal $T$-order
Minimal sporadic ideal

$p_{i}=\tau^{-i}(p)$ for a point $p$

2076, 2080

2071

2076, 2077

$P(p), P^{\prime}(p)$

2064

2063

2076

2099

2075

2109 semigraded morphism

2067,2101

2062

Sporadic ideal

eaves

2079

2076

2064

2076

$(\boldsymbol{d})$, effective blowup

TCR, twisted coordinate ring $B(X, \mathscr{L}, \theta)$

\section{1}

Unregraded ring

2062

al blowup

2095

Virtually effective divisor $\boldsymbol{x}=\boldsymbol{u}-\boldsymbol{v}+\tau^{-1}(\boldsymbol{v})$

Normalised orbit representative, divisor $\widehat{X}, \bar{X}$

2095

$2082 \doteq$, equal in high degree
2064

2069

\section{References}

[Artin 1997] M. Artin, "Some problems on three-dimensional graded domains", pp. 1-19 in Representation theory and algebraic geometry (Waltham, MA, 1995), edited by A. Martsinkovsky and G. Todorov, London Math. Soc. Lecture Note Ser. 238, Cambridge Univ. Press, 1997. MR 99a:16037 Zbl 0888.16025

[Artin and Stafford 1995] M. Artin and J. T. Stafford, "Noncommutative graded domains with quadratic growth”, Invent. Math. 122:2 (1995), 231-276. MR 96g:16027 Zbl 0849.16022

[Artin and Van den Bergh 1990] M. Artin and M. Van den Bergh, "Twisted homogeneous coordinate rings", J. Algebra 133:2 (1990), 249-271. MR 91k:14003 Zbl 0717.14001

[Artin and Zhang 1994] M. Artin and J. J. Zhang, "Noncommutative projective schemes", Adv. Math. 109:2 (1994), 228-287. MR 96a:14004 Zbl 0833.14002

[Artin et al. 1990] M. Artin, J. Tate, and M. Van den Bergh, "Some algebras associated to automorphisms of elliptic curves", pp. 33-85 in The Grothendieck Festschrift, I, edited by P. Cartier et al., Progr. Math. 86, Birkhäuser, Boston, 1990. MR 92e:14002 Zbl 0744.14024 
[Artin et al. 1991] M. Artin, J. Tate, and M. Van den Bergh, "Modules over regular algebras of dimension 3", Invent. Math. 106:2 (1991), 335-388. MR 93e:16055 Zbl 0763.14001

[Artin et al. 1999] M. Artin, L. W. Small, and J. J. Zhang, "Generic flatness for strongly Noetherian algebras”, J. Algebra 221:2 (1999), 579-610. MR 2001a:16006 Zbl 0958.16024

[Björk and Ekström 1990] J.-E. Björk and E. K. Ekström, "Filtered Auslander-Gorenstein rings", pp. 425-448 in Operator algebras, unitary representations, enveloping algebras, and invariant theory (Paris, 1989), edited by A. Connes et al., Progr. Math. 92, Birkhäuser, Boston, 1990. MR 92d:16017 Zbl 0733.16016

[Cozzens 1976] J. H. Cozzens, "Maximal orders and reflexive modules", Trans. Amer. Math. Soc. 219 (1976), 323-336. MR 54 \#7524 Zbl 0334.16009

[Etingof and Ginzburg 2010] P. Etingof and V. Ginzburg, "Noncommutative del Pezzo surfaces and Calabi-Yau algebras", J. Eur. Math. Soc. (JEMS) 12:6 (2010), 1371-1416. MR 2012d:16082 Zbl 1204.14004

[Goodearl and Warfield 1989] K. R. Goodearl and R. B. Warfield, Jr., An introduction to noncommutative Noetherian rings, London Math. Soc. Student Texts 16, Cambridge Univ. Press, 1989. MR 91c:16001 Zbl 0679.16001

[Hartshorne 1977] R. Hartshorne, Algebraic geometry, Graduate Texts in Mathematics 52, Springer, New York, 1977. MR 57 \#3116 Zbl 0367.14001

[Keeler et al. 2005] D. S. Keeler, D. Rogalski, and J. T. Stafford, "Naïve noncommutative blowing up", Duke Math. J. 126:3 (2005), 491-546. MR 2006g:14005 Zbl 1082.14003

[Krause and Lenagan 1985] G. R. Krause and T. H. Lenagan, Growth of algebras and GelfandKirillov dimension, Research Notes in Mathematics 116, Pitman, Boston, 1985. MR 86g:16001 Zbl 0564.16001

[Levasseur 1992] T. Levasseur, "Some properties of noncommutative regular graded rings", Glasgow Math. J. 34:3 (1992), 277-300. MR 93k:16045 Zbl 0824.16032

[McConnell and Robson 2001] J. C. McConnell and J. C. Robson, Noncommutative Noetherian rings, Revised ed., Graduate Studies in Mathematics 30, American Mathematical Society, Providence, RI, 2001. MR 2001i:16039 Zbl 0980.16019

[Năstăsescu and van Oystaeyen 1982] C. Năstăsescu and F. van Oystaeyen, Graded ring theory, NorthHolland Mathematical Library 28, North-Holland Publishing Co., Amsterdam, 1982. MR 84i:16002 Zbl 0494.16001

[Rogalski 2011] D. Rogalski, "Blowup subalgebras of the Sklyanin algebra", Adv. Math. 226:2 (2011), 1433-1473. MR 2012d:16083 Zbl 1207.14004

[Rogalski and Sierra 2012] D. Rogalski and S. J. Sierra, "Some projective surfaces of GK-dimension 4”, Compos. Math. 148:4 (2012), 1195-1237. MR 2956041 Zbl 1262.16024

[Rogalski and Stafford 2009] D. Rogalski and J. T. Stafford, "A class of noncommutative projective surfaces”, Proc. Lond. Math. Soc. (3) 99:1 (2009), 100-144. MR 2010j:14007 Zbl 1173.14005

[Rotman 2009] J. J. Rotman, An introduction to homological algebra, 2nd ed., Springer, New York, 2009. MR 2009i:18011 Zbl 1157.18001

[RSS 2014] D. Rogalski, S. J. Sierra, and J. T. Stafford, "Algebras in which every subalgebra is Noetherian”, Proc. Amer. Math. Soc. 142:9 (2014), 2983-2990. MR 3223353 Zbl 1311.16015

[RSS 2015] D. Rogalski, S. J. Sierra, and J. T. Stafford, "Noncommutative blowups of elliptic algebras”, Algebr. Represent. Theory 18:2 (2015), 491-529. MR 3336351 Zbl 06445654

[Sierra 2011] S. J. Sierra, "Classifying birationally commutative projective surfaces", Proc. Lond. Math. Soc. (3) 103:1 (2011), 139-196. MR 2012i:16054 Zbl 1226.14004 
[Stafford and Van den Bergh 2001] J. T. Stafford and M. Van den Bergh, "Noncommutative curves and noncommutative surfaces”, Bull. Amer. Math. Soc. (N.S.) 38:2 (2001), 171-216. MR 2002d:16036 Zbl 1042.16016

[Stafford and Zhang 1994] J. T. Stafford and J. J. Zhang, "Examples in non-commutative projective geometry", Math. Proc. Cambridge Philos. Soc. 116:3 (1994), 415-433. MR 95h:14001 Zbl 0821.16026

[Stephenson 1997] D. R. Stephenson, "Algebras associated to elliptic curves", Trans. Amer. Math. Soc. 349:6 (1997), 2317-2340. MR 97m:16080 Zbl 0868.16028

[Van den Bergh 1997] M. Van den Bergh, "Existence theorems for dualizing complexes over non-commutative graded and filtered rings", J. Algebra 195:2 (1997), 662-679. MR 99b:16010 Zbl 0894.16020

[Van den Bergh 2001] M. Van den Bergh, Blowing up of non-commutative smooth surfaces, Mem. Amer. Math. Soc. 734, Amer. Math. Soc., Providence, 2001. MR 2002k:16057 Zbl 0998.14002

[Van den Bergh 2011] M. Van den Bergh, "Noncommutative quadrics", Int. Math. Res. Not. 2011:17 (2011), 3983-4026. MR 2012m:14004 Zbl 1311.14003

[Van den Bergh 2012] M. Van den Bergh, "Non-commutative $\mathbb{P}^{1}$-bundles over commutative schemes", Trans. Amer. Math. Soc. 364:12 (2012), 6279-6313. MR 2958936 Zbl 06199600

[Zhang 1998] J. J. Zhang, "On lower transcendence degree”, Adv. Math. 139:2 (1998), 157-193. MR 2000a:16046 Zbl 0924.16015

Communicated by Efim Zelmanov

Received 2014-04-08 Revised 2015-03-10 Accepted 2015-09-03

drogalsk@math.ucsd.edu Department of Mathematics, University of California, San Diego, 9500 Gilman Drive \#0112, La Jolla, CA 92093-0112, United States

s.sierra@ed.ac.uk

toby.stafford@manchester.ac.uk
School of Mathematics, University of Edinburgh, James Clerk Maxwell Building, Peter Guthrie Tait Road, Edinburgh, EH9 3FD, United Kingdom

School of Mathematics, University of Manchester, Alan Turing Building, Oxford Road, Manchester, M13 9PL, United Kingdom 


\section{Algebra \& Number Theory}

msp.org/ant

\section{EDITORS}

MANAGING EDITOR

Bjorn Poonen

Massachusetts Institute of Technology

Cambridge, USA

\author{
EDITORIAL BOARD CHAIR \\ David Eisenbud \\ University of California \\ Berkeley, USA
}

BOARD OF EDITORS

Georgia Benkart

Dave Benson

Richard E. Borcherds

John H. Coates

J-L. Colliot-Thélène

Brian D. Conrad

Hélène Esnault

Hubert Flenner

Sergey Fomin

Edward Frenkel

Andrew Granville

Joseph Gubeladze

Roger Heath-Brown

Craig Huneke

Kiran S. Kedlaya

János Kollár

Yuri Manin

Philippe Michel
University of Wisconsin, Madison, USA

University of Aberdeen, Scotland

University of California, Berkeley, USA

University of Cambridge, UK

CNRS, Université Paris-Sud, France

Stanford University, USA

Freie Universität Berlin, Germany

Ruhr-Universität, Germany

University of Michigan, USA

University of California, Berkeley, USA

Université de Montréal, Canada

San Francisco State University, USA

Oxford University, UK

University of Virginia, USA

Univ. of California, San Diego, USA

Princeton University, USA

Northwestern University, USA

École Polytechnique Fédérale de Lausanne
Susan Montgomery

Shigefumi Mori

Raman Parimala

Jonathan Pila

Anand Pillay

Victor Reiner

Peter Sarnak

Joseph H. Silverman

Michael Singer

Vasudevan Srinivas

J. Toby Stafford

Ravi Vakil

Michel van den Bergh

Marie-France Vignéras

Kei-Ichi Watanabe

Efim Zelmanov

Shou-Wu Zhang
University of Southern California, USA

RIMS, Kyoto University, Japan

Emory University, USA

University of Oxford, UK

University of Notre Dame, USA

University of Minnesota, USA

Princeton University, USA

Brown University, USA

North Carolina State University, USA

Tata Inst. of Fund. Research, India

University of Michigan, USA

Stanford University, USA

Hasselt University, Belgium

Université Paris VII, France

Nihon University, Japan

University of California, San Diego, USA

Princeton University, USA

\section{PRODUCTION}

production@msp.org

Silvio Levy, Scientific Editor

See inside back cover or msp.org/ant for submission instructions.

The subscription price for 2015 is US $\$ 255 /$ year for the electronic version, and $\$ 440 /$ year ( $+\$ 55$, if shipping outside the US) for print and electronic. Subscriptions, requests for back issues and changes of subscribers address should be sent to MSP.

Algebra \& Number Theory (ISSN 1944-7833 electronic, 1937-0652 printed) at Mathematical Sciences Publishers, 798 Evans Hall \#3840, c/o University of California, Berkeley, CA 94720-3840 is published continuously online. Periodical rate postage paid at Berkeley, CA 94704, and additional mailing offices.

ANT peer review and production are managed by EditFLOW ${ }^{\circledR}$ from MSP.

\section{PUBLISHED BY}

- mathematical sciences publishers

nonprofit scientific publishing

http://msp.org/

(C) 2015 Mathematical Sciences Publishers 


\section{Algebra \& Number Theory}

Volume $9 \quad$ No. $9 \quad 2015$

Families of nearly ordinary Eisenstein series on unitary groups XIN WAN

Classifying orders in the Sklyanin algebra

Daniel Rogalski, Susan J. SierRa and J. Toby STAFFord

Chongying Dong, XingJun Lin and SiU-Hung NG

KAISA MATOMÄKI, MAKSYM RADZIWIŁŁ and TERENCE TAO 